\title{
Electron transmission through atom-contacted carbon nanotubes
}

\author{
L. F. Chibotaru, ${ }^{*}$ S. Compernolle, and A. Ceulemans \\ Department of Chemistry, University of Leuven, Celestijnenlaan 200F, B-3001 Leuven, Belgium
}

(Received 21 December 2002; revised manuscript received 23 June 2003; published 24 September 2003)

\begin{abstract}
The transmission through side-contacted single-wall carbon nanotubes is investigated within the LandauerBütikker formalism for arbitrary tubes, geometries of contacts, and energies of transmission $(E)$ for the case of monoatomic contacts. An efficient method to calculate transmission within the tight-binding approach including curvature effects is devised allowing, in particular, for an analytical treatment of zigzag, armchair, and some chiral tubes. The transmission function is described as a superposition of the contributions from onedimensional bands of all nanotube lines, each of them oscillating or/and decaying with the distance between the leads. We find five different types of antiresonances. Two of them arise from a destructive interference of the contributions from different nanotube lines when $E$ is in the gap of semiconducting nanotubes and either are due to symmetry reasons or are accidental. A further two can show up at energies of Van Hove singularities originating from isolated singular points in 1D bands of nanotubes and can lead either to split or completely missing transmission peaks. The fifth one is related to the flat band in zigzag nanotubes at the energy of Van Hove singularity in graphene and results in zero transmission between carbon atoms belonging to different translational units in these tubes. A strong anisotropy of transmission in two opposite directions along the tube's axis is found in the middle of the energy gap of semiconducting nanotubes, which reaches several orders of magnitude in zigzag tubes. The transmission at Van Hove singularities (when nonzero) is of the order of unity and decays at a large axial distance $R$ between the leads as $R^{-2}$, similarly to monoatomic chains. The transmission in the band region of nanotubes shows two types of behavior depending on the divisibility by three of the difference between corresponding reduced nanotube indices $n^{\prime}-m^{\prime}$. Close to the Fermi level the amplitude of the transmission function is independent from the chiral angle of the nanotubes and scales as an inverse square of their diameter.
\end{abstract}

DOI: 10.1103/PhysRevB.68.125412

PACS number(s): 73.23.Ad, 73.63.Fg

\section{INTRODUCTION}

Recent experimental investigations ${ }^{1-4}$ of the conductivity of carbon nanotubes prompted intensive theoretical research of this new family of molecular conductors. ${ }^{5-13}$ It was found that carbon nanotubes possess a unique combination of mechanical and electronic properties making them best suited as molecular conductors in molecular electronic devices applications. ${ }^{14-16}$ Being stiff and elastic ${ }^{17-19}$ and almost free of defects on a macroscopic scale, they show ballistic conductance ${ }^{2,3}$ which is quite stable against deformations $^{20-23}$ and disorder. ${ }^{9,10,24}$ In addition, carbon nanotubes have been shown to transmit electronic currents of very high density. ${ }^{3}$

The conductance is usually measured in two basic geometries of contacts. In end-contacted nanotubes the leads are attached to the ends of the tubes. In this geometry, a strong interaction between metal and carbon atoms is realized resulting in low contact resistance. In side-contacted nanotubes the leads are attached to their cylindrical surface. Contrary to the previous case the interaction between metal and carbon atoms is weak. Experimentally both geometries of contacts have been achieved. At the same time most theoretical investigations have been devoted to end-contacted nanotubes. ${ }^{5-13,21,22,25}$ For defect-free single-wall carbon nanotubes with reflectionless contacts the Landauer theory ${ }^{26}$ predicts a value of $2 G_{0}$ for the low bias ballistic conductance, where $G_{0}=2 e^{2} / h$ is the conductance quantum. This value of conductance is explained by the presence of two transmitting channels at the Fermi level of metallic nano- tubes. Actually the conductance is lowered by nonideal contacts and sources of scattering in both single- and multiwall nanotubes ${ }^{9,13}$ but still remains of the order of $G_{0}$ for very long tubes and for a wide range of energies of transmission. On the other hand in the side-contact geometry the transmission is determined to a greater extent by the details of the electronic structure of nanotubes. For large enough contacts this is manifested, particularly, in the wave vector conservation effects in the transmission of an electron from metal to the nanotube..$^{27,28}$

The scope of this paper is the comprehensive study of the electron transmission through infinite single-wall nanotubes which are contacted by two leads attached at arbitrary positions on their cylindrical surface. The emphasis is put on revealing the influence of the intrinsic electronic and topological properties of the nanotubes on the transmission. For this purpose we only consider single-atomic contacts but treat in a transparent analytical way the contributions from different transmission channels and their interference in the final transmission amplitude. Such an analysis provides complete understanding of the origin of various features of the transmission function and complements many numerical investigations of the nanotubes undertaken so far. $^{5-9,12,13,21,28-33}$

The paper is organized as follows. Section II begins with an overview of the electronic structure of carbon nanotubes. Then it describes the theoretical approach for the transmission and, with Appendixes A and B, the derivation of the analytical expressions for the Green's functions of the nanotubes. Section III presents the analysis of the transmission 
through zigzag, armchair and chiral nanotubes as function of the geometry of contacts and the energies of transmission. Appendixes C and D contain the main analytical derivations used in this section. Section IV gives the discussion of the features of the formalism, the key results for the sidecontacted transmission, the summary and perspectives for the future research.

\section{MODEL AND METHOD}

An infinite single-wall carbon nanotube is fully defined by the chiral vector $\mathbf{C}_{h}=n \mathbf{a}_{1}+m \mathbf{a}_{2}$, Fig. 1(a), connecting the edges of the strip cut from a graphene plane. Accordingly each nanotube is specified by the corresponding pair of indices $(n, m) .{ }^{34}$ The chiral angle $\theta$ can take independent values in the range $0<\theta<\pi / 6$. The orbital eigenstates of the nanotubes are just graphene wavefunctions for specific values of $\mathbf{k}$ vectors from the Brillouin zone of graphene:

$$
|\mu \mathbf{k}\rangle=\frac{1}{\sqrt{N}} \sum_{\mathbf{t}} e^{i \mathbf{k} \cdot \mathbf{t}}\left(c_{\mu \mathbf{k}}^{1}|\mathbf{t}\rangle_{1}+c_{\mu \mathbf{k}}^{2}|\mathbf{t}\rangle_{2}\right)
$$

where the summation extends over all unit cells $\mathbf{t}$ within the strip, $N$ is the total number of unit cells, $|\mathbf{t}\rangle_{1}$ and $|\mathbf{t}\rangle_{2}$ are $2 p_{z}$ orbitals centered on the atoms 1 and 2 of the graphene's unit cell $\mathbf{t}$ [Fig. 1(a)] and $\mu$ is the band index $(\mu=1,2)$. Given the bipartite character of the graphene lattice, the mixing coefficients $c_{\mu \mathbf{k}}^{1}$ and $c_{\mu \mathbf{k}}^{2}$ in Eq. (1) are of the same absolute value:

$$
c_{\mu \mathbf{k}}^{1}=\sigma_{\mu} \frac{f_{\mathbf{k}}}{\left|f_{\mathbf{k}}\right|} \frac{1}{\sqrt{2}}, \quad c_{\mu \mathbf{k}}^{2}=\frac{1}{\sqrt{2}},
$$

where $\sigma_{1}=1$ and $\sigma_{2}=-1 . f_{\mathbf{k}}$ depends on the details of the $\pi$-electron Hamiltonian and is therefore different for graphene and nanotubes. Indeed, in graphene the electron transfer between the $2 p_{z}$ orbitals of the nearest neighbor atoms is described within the tight-binding approximation by one single transfer parameter $\gamma_{0} \approx-2.8 \mathrm{eV}$. In a nanotube, due to the curvature of the graphene plane in the $\mathbf{C}_{h}$ direction all three nearest neighbors of a given carbon atom are generally nonequivalent, which results in three different transfer parameters $\gamma_{1}, \gamma_{2}$, and $\gamma_{3}$ [Fig. 1(a)]. Symmetry relations between these parameters only exist for armchair $\left(\gamma_{2}\right.$
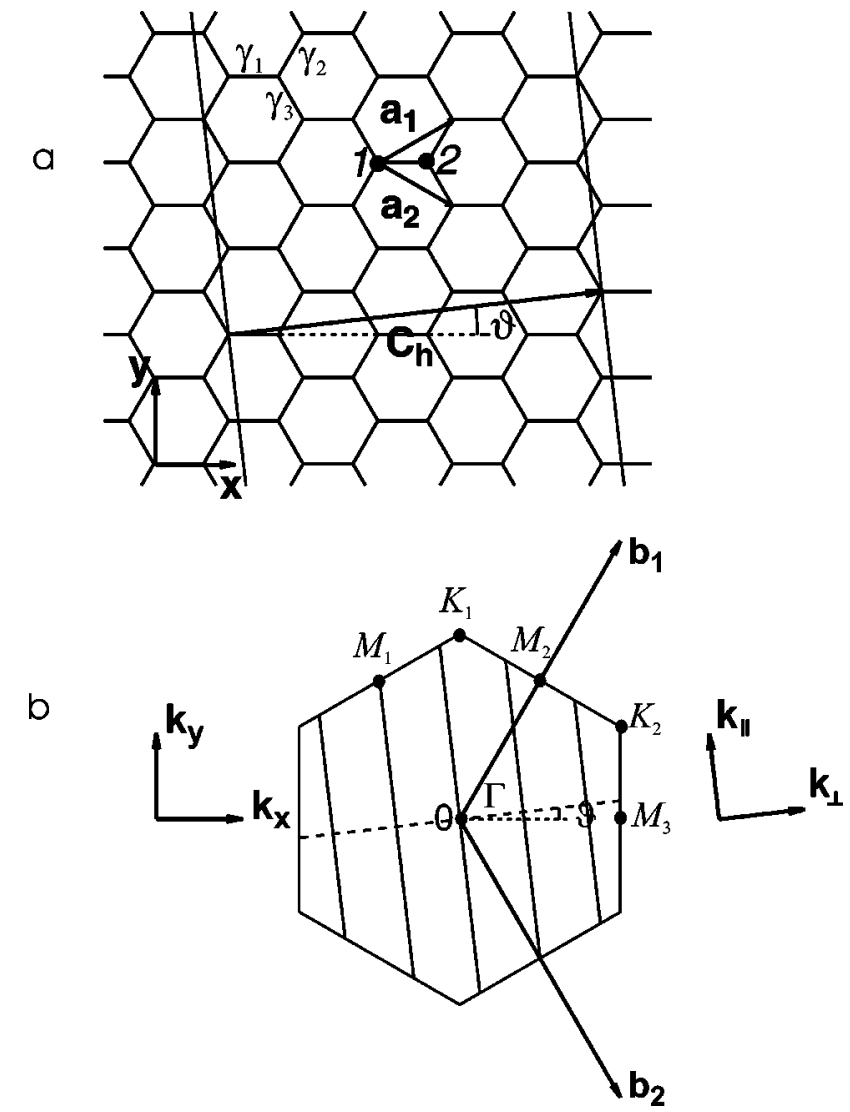

FIG. 1. (a) $\mathbf{a}_{1}$ and $\mathbf{a}_{2}$ are unit vectors and bold sites 1 and 2 denote two carbon atoms of the graphite's unit cell. The fine line delineates the strip corresponding to an unrolled $(3,2)$ nanotube and $\gamma_{1}, \gamma_{2}$ and $\gamma_{3}$ are transfer parameters corresponding to three nonequivalent bonds. (b) The first (hexagonal) Brillouin zone of a graphite sheet. $\mathbf{b}_{1}$ and $\mathbf{b}_{2}$ are unit vectors of the reciprocal lattice corresponding to $\mathbf{a}_{1}$ and $\mathbf{a}_{2}$. Parallel lines correspond to "allowed" $\mathbf{k}$ states in a $(3,2)$ nanotube.

$\left.=\gamma_{3}, \theta=0\right)$ and zigzag $\left(\gamma_{1}=\gamma_{2}, \theta=\pi / 6\right)$ nanotubes. For a general case of a chiral tube the function $f_{\mathbf{k}}$ has the form

$$
f_{\mathbf{k}}=\gamma_{1}+\gamma_{2} e^{-i \mathbf{k} \cdot \mathbf{a}_{1}}+\gamma_{3} e^{-i \mathbf{k} \cdot \mathbf{a}_{2}}
$$

while the energy corresponding to the $|\mu \mathbf{k}\rangle$ state is given by

$$
E_{\mu \mathbf{k}}=\alpha+\sigma_{\mu} \sqrt{\gamma_{1}^{2}+\gamma_{2}^{2}+\gamma_{3}^{2}+2 \gamma_{1} \gamma_{2} \cos \left(\sqrt{3} k_{x}+k_{y}\right) \frac{a}{2}+2 \gamma_{1} \gamma_{3} \cos \left(\sqrt{3} k_{x}-k_{y}\right) \frac{a}{2}+2 \gamma_{2} \gamma_{3} \cos k_{y} a},
$$

where $\alpha$ is the electron energy in the $2 p_{z}$ orbital and $a$ $\equiv a_{1}=a_{2}$. For tubes with large enough diameters [such as, e.g., $(10,10)$ ones], when the tight-binding $\pi$ approximation can be applied, the parameters $\gamma_{i}$ differ only slightly from $\gamma_{0}$. Neglecting this difference will recover of course the graphene's expressions ${ }^{35}$ for Eqs. (3) and (4).
The periodic boundary conditions along $\mathbf{C}_{h}$ imply quantization of the $\mathbf{k}$ vector in this direction:

$$
k_{\perp}=\frac{2 \pi l}{C_{h}}
$$


where $l$ is an integer and $C_{h}=\sqrt{\left(n^{2}+m^{2}+n m\right)} a$ is the width of the strip. In contrast the components in the direction of the tube's axis $k_{\|}$remain continuous so that the set of allowed $\mathbf{k}$ vectors form equidistant parallel lines in the Brillouin zone of graphene spaced by $2 \pi / C_{h}$ [Fig. 1(b)]. The angle these nanotube lines make with the $k_{y}$ axis of the graphene Brillouin zone is equal to the chiral angle $\theta$ in Fig. 1(a). The $\Gamma$ point of the graphene Brillouin zone is always intersected by one nanotube line. The other two important points $K_{1}$ and $K_{2}$, are the only ones where the two $\pi$ bands of the graphene get in touch. These points are intersected by the nanotubes lines of all armchair tubes, zigzag tubes, $(n, 0)$, with $n$ multiple to 3 , and chiral tubes, $(n, m)$, with $n-m$ multiple to 3 . Such tubes are called primary metallic. Actually due to the curvature of the surface, and the related nonequivalency of the transfer parameters $\gamma_{1}, \gamma_{2}$, and $\gamma_{3}$, only the armchair tubes remain "metallic", i.e., keep the degeneracy of the $\pi$ bands in the $K$ points, all others becoming semiconducting. ${ }^{20}$

\section{A. Conductivity and transmission}

We investigate the coherent conductance within the Landauer-Bütikker formalism, ${ }^{36}$ described by the electron transmission from lead 1 to lead 2 , evaluated from the Fisher-Lee relation ${ }^{36}$

$$
T(E)=\operatorname{tr}\left[\Gamma_{1} G_{c}^{r} \Gamma_{2} G_{c}^{a}\right],
$$

where $G_{c}^{r}(E)$ and $G_{c}^{a}(E)$ are retarded and advanced Green's functions of the conductor. In the site representation they obey the matrix equation

$$
\begin{gathered}
{\left[z I-H_{c}-V_{c}-\Sigma_{1}^{r}-\Sigma_{2}^{r}\right] G_{c}^{r}(E)=I,} \\
G_{c}^{a}(E)=G_{c}^{r}(E)^{\dagger},
\end{gathered}
$$

where $z=E+i \eta\left(\eta \rightarrow 0^{+}\right), I$ is a unity matrix defined in the space of orthogonal $\pi$ orbitals localized at the carbon atoms of the nanotube, $H_{c}$ is the corresponding tight-binding Hamiltonian of an isolated nanotube, which includes also curvature effects discussed above, and $V_{c}$ is an operator describing possible defects and other perturbations of the nanotube. $\Sigma_{1}^{r}(E)$ and $\Sigma_{2}^{r}(E)$ are retarded self-energy functions ${ }^{36}$ due to the interaction with the first and the second lead and the interaction matrices $\Gamma_{1}$ and $\Gamma_{2}$ in Eq. (6) are twice their imaginary parts.

In this paper we will be concerned with simple contacts when only few carbon atoms of the nanotube become perturbed. Then it is convenient to rewrite Eq. (7) in the form (all functions are retarded)

$$
G(E)^{-1}=G^{0}(E)^{-1}-V_{c}-\Sigma_{1}-\Sigma_{2}
$$

containing explicitly the Green's function for the isolated nanotube which obeys the equation

$$
\left[z I-H_{0}\right] G^{0}(E)=I .
$$

The solution of Eq. (8) can be written as a Dyson equation

$$
G=\left[I-G^{0}\left(V_{c}+\Sigma_{1}+\Sigma_{2}\right)\right]^{-1} G^{0} .
$$

The advantage of this approach is that once having solved the (infinite dimension) matrix equation (9) for $G^{0}$, one can easily obtain $G$ for any particular problem by just inserting in Eq. (10) the appropriate matrices for the perturbation operator and self-energies. The important point is that we have to invert in Eq. (10) a matrix block of only a finite dimension. Indeed, the calculation of transmission by Eq. (6) requires matrix elements of Green's functions only between the $\pi$ orbitals of the carbon atoms which are in contact with the leads. For such matrix elements of $G$, one can prove (e.g., by a straightforward Taylor expansion of the inverse matrix) that the right-hand side of Eq. (10) will contain matrix elements of $G^{0}$ only between the orbitals of perturbed carbon atoms, i.e., those in direct contact with the leads and affected by the additional perturbation $V_{c}$. Hence to perform inversion in Eq. (10) we can a priori confine ourselves with the matrix block of the operator in the square brakets, which is defined in the space of above orbitals only.

The calculation of transmission in Eq. (6) requires knowledge of self-energies which depend on the details of electronic structure of the leads. Since our goal is to display the electronic features of nanotubes in transmission we will try to treat self-energies as simple as possible. Following Ref. 37 we assume the Green's functions of the leads to be diagonal matrices and energy independent in a wide a range of energies around the Fermi level. This is partly justified by the observation of an almost constant local DOS around the Fermi energy of gold. ${ }^{37,38}$ Then the corresponding real part of self-energy will be zero since it is obtained via KramersKrönig transformation of the dispersionless imaginary part. It was found that such a simplified treatment of self-energy compares well with detailed calculations of conduction for some molecules. ${ }^{37}$ Another example where the real part of self-energy drops out ${ }^{39}$ corresponds to the transmission via the leads having the spectral density as in the AndersonNewns chemisorption theory ${ }^{40}$ at the Fermi energy of half filled bands.

\section{B. Green's functions for single-wall nanotubes}

The eigenfunction expansion ${ }^{41}$ of the Green's functions of single-wall nanotubes in the site representation has the form

$$
G^{0}(\Delta \mathbf{t}, r s ; E)=\sum_{\mu, \mathbf{k}} \frac{{ }_{r}\left[\mathbf{t}_{i}|\mu \mathbf{k}\rangle\left\langle\mu \mathbf{k} \mid \mathbf{t}_{j}\right\rangle_{s}\right.}{z-E_{\mu \mathbf{k}}}
$$

where $|\mu \mathbf{k}\rangle$ and $E_{\mu \mathbf{k}}$ are the eigenfunctions and eigenenergies of nanotubes given by Eqs. (1) and (4) respectively, $\mathbf{t}_{i}$ and $\mathbf{t}_{j}$ denote the unit cells, $\Delta \mathbf{t}=\mathbf{t}_{i}-\mathbf{t}_{j}$, and $r$ and $s$ denote the two carbon atoms in each unit cell [Fig. 1(a)]. Substituting Eqs. $(1,2)$ into $(11)$ one obtains

$$
G^{0}(\Delta \mathbf{t}, 11 ; E)=\frac{1}{2 N} \sum_{\mu, \mathbf{k}} \frac{e^{i \mathbf{k} \Delta \mathbf{t}}}{z-E_{\mu, \mathbf{k}}}
$$

$$
G^{0}(\Delta \mathbf{t}, 12 ; E)=\frac{1}{2 N} \sum_{\mu, \mathbf{k}} \sigma_{\mu} \frac{f_{\mathbf{k}}}{\left|f_{\mathbf{k}}\right|} \frac{e^{i \mathbf{k} \Delta \mathbf{t}}}{z-E_{\mu, \mathbf{k}}}
$$


Choosing the $\pi$-electron orbital energy $\alpha$ in expression (4) for $E_{\mu, \mathbf{k}}$ as zero of energy, the summations in Eq. (12) can be confined to one single band:

$$
\begin{aligned}
& G^{0}(\Delta \mathbf{t}, 11 ; E)=\frac{z}{N} \sum_{\mathbf{k}} \frac{e^{i \mathbf{k} \Delta \mathbf{t}}}{z^{2}-E_{\mathbf{k}}^{2}}, \\
& G^{0}(\Delta \mathbf{t}, 12 ; E)=\frac{1}{N} \sum_{\mathbf{k}} \frac{f_{\mathbf{k}} e^{i \mathbf{k} \Delta \mathbf{t}}}{z^{2}-E_{\mathbf{k}}^{2}},
\end{aligned}
$$

where we dropped $\mu$ from the subscript of $E_{\mathbf{k}}$. Taking into account the invariance of the band structure under the inversion in the $\mathbf{k}$ space and the equivalence of all carbon atoms in nanotubes ${ }^{42}$ one obtains the following relations between matrix elements of the Green's function:

$$
\begin{gathered}
G^{0}(\Delta \mathbf{t}, 22 ; E)=G^{0}(\Delta \mathbf{t}, 11 ; E), \\
G^{0}(\Delta \mathbf{t}, 11 ; E)=G^{0}(-\Delta \mathbf{t}, 11 ; E), \\
G^{0}(\Delta \mathbf{t}, 12 ; E)=G^{0}(-\Delta \mathbf{t}, 21 ; E) .
\end{gathered}
$$

The summations in Eqs. (13) are further split into two independent sums running over the discrete $\left(k_{\perp}\right)$ and continuous $\left(k_{\|}\right)$components of $\mathbf{k}$. Using Eq. (5) and passing to integration over $k_{\|}$, yields for Eqs. (13)

$$
\begin{gathered}
G^{0}(\Delta \mathbf{t}, 11 ; E)=z \frac{\sqrt{3} a^{2}}{4 \pi C_{h}} \sum_{l} e^{i \Delta k_{\perp} l \Delta t_{\perp}} \int_{k_{\|}} \frac{e^{i k_{\|} \Delta t_{\|}}}{z^{2}-E_{k_{\|} l}^{2}} d k_{\|}, \\
G^{0}(\Delta \mathbf{t}, 12 ; E)=\frac{\sqrt{3} a^{2}}{4 \pi C_{h}} \sum_{l} e^{i \Delta k_{\perp} l \Delta t_{\perp}} \int_{k_{\|}} f_{k_{\|} l} \frac{e^{i k_{\|} \Delta t \|}}{z^{2}-E_{k_{\|} l}^{2} l} d k_{\|},
\end{gathered}
$$

where $\Delta k_{\|}=2 \pi / C_{h}$ and the composite index $k_{\|} l$ is used for k. $\Delta t_{\|}$and $\Delta t_{\perp}$ are the components of the vector $\mathbf{t}$ in the axial and circumferential directions of the nanotube, which are expressed through Cartesian components as follows:

$$
\begin{aligned}
& \Delta t_{\|}=\frac{\Delta t_{x}\left(m^{\prime}-n^{\prime}\right)+\sqrt{3} \Delta t_{y}\left(n^{\prime}+m^{\prime}\right)}{2 \sqrt{n^{\prime 2}+m^{\prime 2}+n^{\prime} m^{\prime}}}, \\
& \Delta t_{\perp}=\frac{\sqrt{3} \Delta t_{x}\left(n^{\prime}+m^{\prime}\right)+\Delta t_{y}\left(n^{\prime}-m^{\prime}\right)}{2 \sqrt{n^{\prime 2}+m^{\prime 2}+n^{\prime} m^{\prime}}} .
\end{aligned}
$$

In this equation the reduced nanotube indices are introduced:

$$
\begin{aligned}
n^{\prime} & =n / \operatorname{GCD}(n, m), \\
m^{\prime} & =m / \operatorname{GCD}(n, m),
\end{aligned}
$$

where $\operatorname{GCD}(n, m)$ stands for the greatest common divisor of $n$ and $m$. According to the definition, $n^{\prime}$ and $m^{\prime}$ cannot be both even.

In Eqs. (15), the summation runs over nanotube lines confined by the Brillouin zone of graphene. For each $l$, the limits of integrations are determined by the points of intersection of the corresponding nanotube line with the frame of the hex-

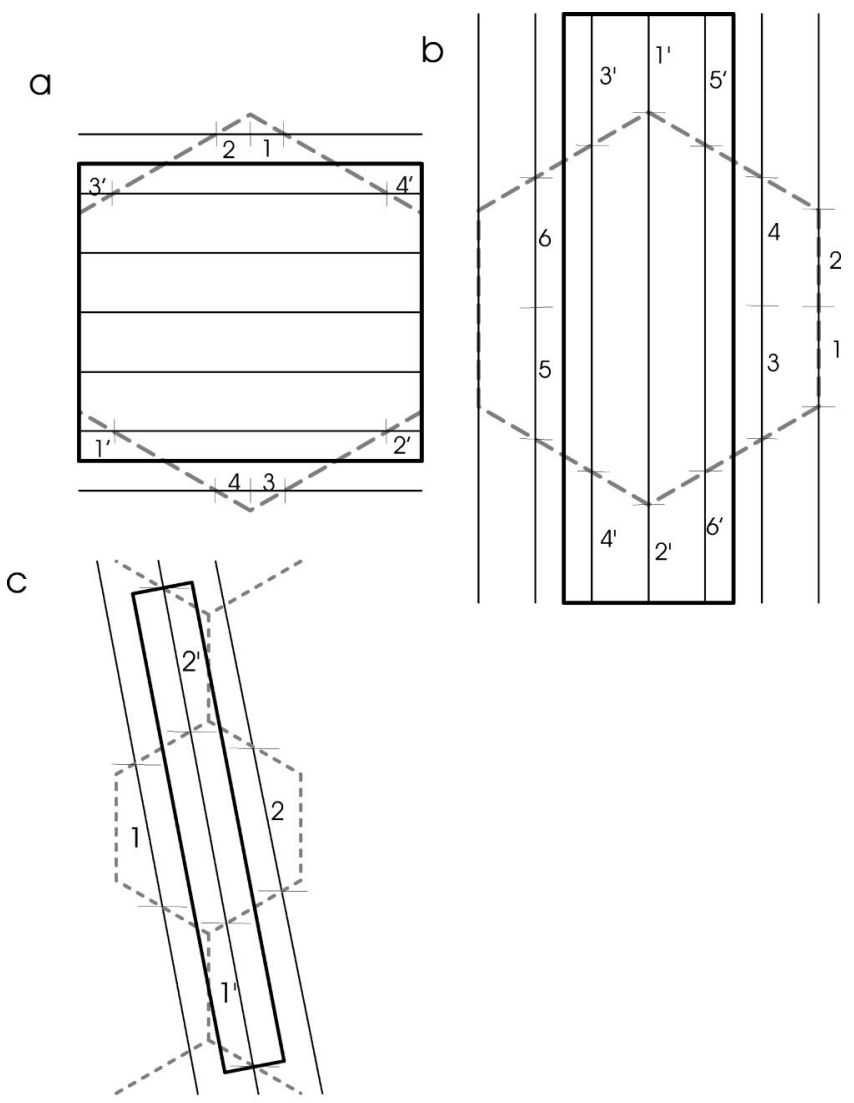

FIG. 2. Conversion of the hexagonal Brillouin zone (dashed lines) into a rectangular-shaped one (bold lines) for $(5,-5)$ zigzag (a), $(3,3)$ armchair (b), and $(2,1)$ chiral (c) nanotubes. Numbered fragments of the nanotube lines of the former Brilloin zone are translated inside the new zone in the positions denoted by corresponding primed numbers.

agonal Brillouin zone of graphene. As Fig. 1(b) shows nanotube lines have different lenghts in a general case. For subsequent calculations it is more convenient to deal with nanotube lines of the same length. This can be achieved by converting the hexagonal Brillouin zone of graphene into a rectangular one using its invariance against translations by arbitrary (integer coefficients) combinations of the unit vectors of the reciprocal space $\mathbf{b}_{1}$ and $\mathbf{b}_{2}$ [Fig. 1(b)]. It is proven in Appendix A that such a rectangular Brillouin zone can always be constructed with edges parallel and perpendicular to the nanotubes lines. These edges are defined by the vectors

$$
\begin{gathered}
\mathbf{W}_{\|}=m^{\prime} \mathbf{b}_{1}-n^{\prime} \mathbf{b}_{2}, \\
\mathbf{W}_{\perp}=\frac{\left(2 n^{\prime}+m^{\prime}\right) \mathbf{b}_{1}+\left(n^{\prime}+2 m^{\prime}\right) \mathbf{b}_{2}}{2\left(n^{\prime 2}+m^{\prime 2}+n^{\prime} m^{\prime}\right)} .
\end{gathered}
$$

The construction of the rectangular Brillouin zone is shown in Fig. 2 for the cases of achiral and chiral nanotubes. This construction can always be done in such a way that the inversion symmetry point of the rectangle coincides with $\mathbf{k}$ $=0$. In this case the allowed values of $k_{\|}$and $l$ are given by 
TABLE I. Integer coordinates defining characteristic vectors $\mathbf{t}$ in nanotubes.

\begin{tabular}{lcc}
\hline \hline$\Delta \mathbf{t}$ & $n_{\|}$ & $n_{\perp}$ \\
\hline $\mathbf{a}_{1}$ & $m^{\prime}$ & $\frac{2 n^{\prime}+m^{\prime}}{R}$ \\
$\mathbf{a}_{2}$ & $-n^{\prime}$ & $\frac{n^{\prime}+2 m^{\prime}}{R}$ \\
$\frac{\mathbf{C}_{h}}{\mathrm{GCD}(\mathrm{n}, \mathrm{m})}$ & 0 & $\frac{2\left(n^{\prime 2}+m^{\prime 2}+n^{\prime} m^{\prime}\right)}{R}$ \\
$\mathbf{T}=\frac{n^{\prime}+2 m^{\prime}}{R} \mathbf{a}_{1}-\frac{2 n^{\prime}+m^{\prime}}{R} \mathbf{a}_{2}$ & $\frac{2\left(n^{\prime 2}+m^{\prime 2}+n^{\prime} m^{\prime}\right)}{R}$ & 0 \\
\hline \hline
\end{tabular}

$-\frac{2 \pi}{\sqrt{3} a} \sqrt{n^{\prime 2}+m^{\prime 2}+n^{\prime} m^{\prime}}<k_{\|} \leqslant \frac{2 \pi}{\sqrt{3} a} \sqrt{n^{\prime 2}+m^{\prime 2}+n^{\prime} m^{\prime}}$

$$
-\frac{\operatorname{GCD}(n, m)}{2}<l \leqslant \frac{\operatorname{GCD}(n, m)}{2},
$$

where the equality in the right-hand side of the second relation takes place only when the greatest common divisor of the nanotube's indices is an even number. In the last case the lines $l= \pm n / 2$ just superimpose over the two edges of the rectangular Brillouin zone. Although the opposite edges parallel to $\mathbf{W}_{\|}$in this Brillouin zone cannot be translated into each other by a lattice vector of the reciprocal space (which is the case for the hexagonal Brillouin zone), they can be superimposed by pieces as it follows from their construction in Fig. 2. Therefore the lines $l= \pm n / 2$ contain equivalent $\mathbf{k}$ points and only one of them should be retained in the folded zone of the nanotube. At the same time the opposite edges corresponding to $\mathbf{W}_{\perp}$ can be translated into each other by $\mathbf{W}_{\|}$ since the last is always a Bravais vector of the reciprocal space. We note in passing that another construction of rectangular Brillouin zone has been proposed recently, ${ }^{43,44}$ in which the edges parallel to the nanotube lines can be translated into each other while the edges perpendicular to the nanotube lines cannot. Such a Brillouin zone represents an opposite case to our construction in Fig. 2 and cannot be used further since we need the end points of each nanotube line to be equivalent points. It also follows from the relation (19) that the rectangular Brillouin zone of $(n, n)$ armchair and $(n,-n)$ zigzag nanotubes contains $n$ lines. On the other hand the chiral tubes $(n, m)$ have much lesser lines in the rectangular Brillouin zone, which reduce even to one single central line (intersecting the point $\mathbf{k}=0$ ) when $n$ and $m$ do not have a common divisor [Fig. 2(c)].

The first relation in Eq. (19) defines the common limits of integration for all nanotube lines in Eqs. (15). Passing to a new variable $\phi$,

$$
k_{\|}=\frac{2 \sqrt{n^{\prime 2}+m^{\prime 2}+n^{\prime} m^{\prime}}}{\sqrt{3} a} \phi,
$$

the limits of integration in Eqs. (15) will become $\pi$ and $-\pi$. On the other hand the Cartesian components of $\Delta \mathbf{t}$ can be expressed through two integers, $n_{x}$ and $n_{y}$, always of the same parity [Fig. 1(a)]:

$$
\Delta t_{x}=\frac{\sqrt{3} a}{2} n_{x}, \quad \Delta t_{y}=\frac{a}{2} n_{y},
$$

with which, using Eq. (16), the axial and circumferential components of the shift vector can be written via corresponding integer coordinates $n_{\|}$and $n_{\perp}$, always of the same parity:

$$
\begin{gathered}
\Delta t_{\|}=\frac{\sqrt{3} a}{2 \sqrt{n^{\prime 2}+m^{\prime 2}+n^{\prime} m^{\prime}}} n_{\|}, \\
\Delta t_{\perp}=\frac{a R}{2 \sqrt{n^{\prime 2}+m^{\prime 2}+n^{\prime} m^{\prime}}} n_{\perp}, \\
n_{\|}=\frac{1}{2}\left[\left(m^{\prime}-n^{\prime}\right) n_{x}+\left(n^{\prime}+m^{\prime}\right) n_{y}\right], \\
n_{\perp}=\frac{1}{2 R}\left[3\left(n^{\prime}+m^{\prime}\right) n_{x}+\left(n^{\prime}-m^{\prime}\right) n_{y}\right],
\end{gathered}
$$

where $R=3$ if $n^{\prime}-m^{\prime}$ is divisible by 3 and $R=1$ otherwise. ${ }^{42}$ Table I shows the values of these coordinates when $\Delta \mathbf{t}$ corresponds to some characteristic vectors of the nanotube. In this table, $\mathbf{T}$ denotes the translational vector along the graphene strip corresponding to a given nanotube, ${ }^{34}$ which is perpendicular to $\mathbf{C}_{h}$ (i.e., parallel to the tube's axis) only for untwisted tubes. ${ }^{45}$ Substituting $\phi, n_{\|}$ and $n_{\perp}$ in expression (15), via Eqs. (20) and (22), we observe that the symmetry of $G^{0}(\Delta \mathbf{t}, 11 ; E)$ with respect to the inversion $\mathbf{t} \rightarrow-\mathbf{t}$ [second relation in Eq. (14)] merely means its invariance under the sign change of both coordinates $n_{\|}$and $n_{\perp}$. We can thus write it in the following form:

$$
\begin{aligned}
G(\Delta \mathbf{t}, 11 ; E)= & \frac{z}{2 \pi \operatorname{GCD}(n, m)} \sum_{l} e^{2 i \pi(l / q) \operatorname{sgn}\left(n_{\|}\right) n_{\perp}} \\
& \times \int_{-\pi}^{\pi} \frac{\exp \left(i \phi\left|n_{\|}\right|\right) \mathrm{d} \phi}{z^{2}-E_{\phi l}^{2}} .
\end{aligned}
$$


On the other hand the matrix elements of the Green's function connecting carbon atoms of different type do not possess such symmetry [see the third relation in Eq. (14)]. Using instead the invariance of these matrix elements with respect to the inversion $\mathbf{k} \rightarrow-\mathbf{k}$ in Eq. (13), which corresponds to a simultaneous transformation of the variables $\phi \rightarrow-\phi, l$ $\rightarrow-l$, we obtain the following expression:

$$
\begin{aligned}
G(\Delta \mathbf{t}, 12 ; E)= & \frac{z}{2 \pi \mathrm{GCD}(n, m)} \sum_{l} \mathrm{e}^{2 i \pi(l / q) \operatorname{sgn}\left(n_{\|}\right) n_{\perp}} \\
& \times \int_{-\pi}^{\pi} \frac{\exp \left(i \phi\left|n_{\|}\right|\right) \mathrm{d} \phi}{z^{2}-E_{\phi l}^{2}} \begin{cases}f_{\phi l} & \text { if } n_{\|} \geqslant 0, \\
f_{\phi l}^{*} & \text { if } n_{\|}<0 .\end{cases}
\end{aligned}
$$

In Eqs. (23) and (24), $q$ denotes the order of the screw axis: ${ }^{42}$

$$
q=2 \operatorname{GCD}(n, m) \frac{\left(n^{\prime 2}+m^{\prime 2}+n^{\prime} m^{\prime}\right)}{R},
$$

defined with respect to the smallest angle of rotation around the tube's axis corresponding to the translation of the graphene's strip by $\mathbf{a}_{1}$ or $\mathbf{a}_{2}$. The ratio on the right-hand side of Eq. (25) is always integer.

The important point is that the $\phi$ dependence of the denominator in the expression under the integral in Eqs. (23), (24) arises only via trigonometric functions of arguments multiple to $\phi$. This enables us to use a procedure employed for analytical calculation of Green's functions of onedimensional chains. ${ }^{41}$ Passing to a complex variable of integration $^{41}$

$$
w=e^{i \phi}
$$

the integral in the above equations is transformed into a contour integral along a circumference of radius $|w|=1$ on the complex plane. The trigonometric functions entering the denominator become ratios of polynomials in $w$, while the denominator $z^{2}-E_{\phi l}^{2}$ itself becomes

$$
-\frac{1}{w^{n^{\prime}+m^{\prime}}} p(w)
$$

where $p(w)$ is a polynomial of degree $2\left(n^{\prime}+m^{\prime}\right)$ of the form

$$
\begin{aligned}
p(w)= & \gamma_{2} \gamma_{3} e^{i\left(d_{\perp}-e_{\perp}\right)} w^{2\left(n^{\prime}+m^{\prime}\right)}+\gamma_{1} \gamma_{3} e^{-i e_{\perp}} w^{2 n^{\prime}+m^{\prime}} \\
& +\gamma_{1} \gamma_{2} e^{i d_{\perp}} w^{n^{\prime}+2 m^{\prime}}+\left(\gamma_{1}^{2}+\gamma_{2}^{2}+\gamma_{3}^{2}-z^{2}\right) w^{n^{\prime}+m^{\prime}} \\
& +\gamma_{1} \gamma_{2} e^{-i d_{\perp}} w^{n^{\prime}}+\gamma_{1} \gamma_{3} e^{i e_{\perp}} w^{m^{\prime}}+\gamma_{2} \gamma_{3} e^{i\left(e_{\perp}-d_{\perp}\right)}
\end{aligned}
$$

and the following notations are introduced:

$$
\begin{aligned}
& d_{\perp}=\frac{\pi l(2 n+m)}{n^{2}+m^{2}+n m}, \\
& e_{\perp}=\frac{\pi l(n+2 m)}{n^{2}+m^{2}+n m} .
\end{aligned}
$$

Equations (23), (24) and the expression (27) are, of course, invariant with respect to the change of nanotube indices induced by the rotation of the graphene strip [Fig. 1(a)] by angles multiple to $\pi / 6$. However the polynomial (28) will not contain negative powers of $w$ only for nanotube indices $n \geqslant m \geqslant 0$, defining the range of nonequivalent chiral angles $0 \leqslant \theta \leqslant \pi / 6$.

Applying the residues theorem for contour integrals, the Green's function matrix element in Eqs. (23), (24) is finally obtained in the form

$$
\begin{aligned}
G^{0}(\Delta \mathbf{t}, 11 ; E)= & -\frac{z}{\operatorname{GCD}(n, m)} \sum_{l} e^{2 i \pi(l / q) \operatorname{sgn}\left(n_{\|}\right) n_{\perp}} \\
& \times \sum_{j} \operatorname{Res}\left[\frac{w^{\left|n_{\|}\right|} w^{n^{\prime}+m^{\prime}-1}}{p(w)}, w_{j}\right]
\end{aligned}
$$

and

$$
\begin{aligned}
G^{0}(\Delta \mathbf{t}, 12 ; E)= & -\frac{1}{\operatorname{GCD}(n, m)} \sum_{l} e^{2 i \pi(l / q) \operatorname{sgn}\left(n_{\|}\right) n_{\perp}} \\
& \times \sum_{j} \operatorname{Res}\left[\frac{w^{\mid n \|} \mid}{p(w)}\left\{\begin{array}{ll}
\left(\gamma_{1} w^{n^{\prime}+m^{\prime}-1}+\gamma_{2} e^{-i d_{\perp}} w^{n^{\prime}-1}+\gamma_{3} e^{-i e_{\perp}} w^{2 n^{\prime}+m^{\prime}-1}\right) & \text { if } n_{\|} \geqslant 0 \\
\left(\gamma_{1} w^{n^{\prime}+m^{\prime}-1}+\gamma_{2} e^{i d_{\perp}} w^{n^{\prime}+2 m^{\prime}-1}+\gamma_{3} e^{i e_{\perp}} w^{m^{\prime}-1}\right) & \text { if } n_{\|}<0
\end{array}\right\}, w_{j}\right],
\end{aligned}
$$

where the second sum runs over the poles, $w_{j}$, which are contained within the circle $|w|<1$. Equations (30) and (31), in combination with the third relation from Eq. (14), allow us to evaluate any matrix element of the Green's function for ideal nanotubes.

We can see that the numerators in Eqs. (30) and (31) are just polynomials, having no poles or branch cuts everywhere on the complex plane $w$. This is a great simplification, not often encountered in multiband problems. ${ }^{46}$ Since the nu- merators are analytic functions, and moreover are linear combinations of terms containing positive powers of $w$ (thanks to the factor $w^{|n|}$ ), the problem of calculating the residues in Eqs. (30),(31) is reduced to finding the zeroes of the polynomial $p(w)$ (28) of the order $2\left(n^{\prime}+m^{\prime}\right)$ in the denominator. Only simple zeroes (and therefore poles) are encountered. In zigzag and armchair nanotubes this polynomial is of the order not higher than four, so the corresponding Green's functions can always be found analytically (Ap- 
pendix B). Generally we can always reduce the order of the polynomial when some relation between its solutions is expected due to the symmetric position of a given nanotube line in the graphene's Brillouin zone. This is the case of the lines $l=0$ and $l=n / 2$ in arbitrary chiral tubes $(f n, g n)$, with $f, g$ nonequal integers different than zero. For instance, the line $l=0$ is always symmetric with respect to the inversion of $k_{\|}$, and from Eqs. (20) and (26) we conclude that half of the solutions are obtained via the inversion of the other half. Therefore the order of the polynomial for independent solutions is reduced twice for this line. As a result, we can find complete analytical solutions for $(2,1)$ and $(4,2)$ nanotubes, reducing the polynomials to the third degree, and for $(3,1)$ and $(6,2)$ nanotubes, reducing the polynomials to the fourth degree. For other nanotubes the above expressions offer a convenient frame for numerical evaluation of Green's functions matrix elements. To conclude, Eqs. (6), (10) and (30), (31) form the basis for the investigation of conduction through side-contacted nanotubes which is carried out with the MATHEMATICA package. ${ }^{49}$

\section{TRANSMISSION THROUGH MONOATOMIC CONTACTS}

Consider two STM tips as leads which contact the nanotube via single carbon atoms. It is assumed that only one atom with an $s$ orbital of each tip is in direct contact with the carbon atoms of the nanotube. This approximation, first introduced in the Tersoff-Hamann theory, ${ }^{50}$ was recently shown to give a reliable description of the differential STM conductance and images of carbon nanotubes. ${ }^{51}$ Supposing equal coupling strength to the carbon atoms for both leads, according to the approximation adopted in Sec. II A, the relevant matrix elements of the self-energy are the following:

$$
\left(\Sigma_{1}\right)_{i i}=\left(\Sigma_{2}\right)_{j j}=-i \Delta,
$$

where $\Delta$ is a positive constant and $i$ and $j$ are composite indices $\left(\mathbf{t}_{i}, r\right)$ and $\left(\mathbf{t}_{j}, s\right)$, denoting carbon atoms in direct contact with the first and the second lead, respectively. As a result the expression for the transmission (6) simplifies to

$$
T=4 \Delta^{2}\left|G_{i j}\right|^{2},
$$

where the Green's function for the nanotube perturbed by the leads is written via Eq. (10) as follows:

$$
G_{i j}=\frac{G_{i j}^{0}}{\left(1+i \Delta G_{11}^{0}\right)^{2}+\Delta^{2} G_{i j}^{02}} .
$$

In these equations, $G_{i j}$ and $G_{i j}^{0}$ stand for $G(\Delta \mathbf{t}, r s ; E)$ and $G^{0}(\Delta \mathbf{t}, r s ; E)$, respectively, $G_{11}^{0} \equiv G^{0}(\mathbf{0}, 11 ; E)=G^{0}(\mathbf{0}, 22 ; E)$ and the relations (14) have been used for the off-diagonal elements of the noninteracting Green's function $G_{i j}^{0}$. Before going to particular nanotubes, let us point out some general features of the transmission function.

\section{A. Properties of the transmission function at singularities}

With Eqs. (33) and (34) the transmission can be written in the form

$$
T=\left|\frac{1}{\frac{1}{2 \Delta G_{i j}^{0}}+i \frac{G_{11}^{0}}{G_{i j}^{0}}-\frac{\Delta}{2} \frac{G_{11}^{02}-G_{i j}^{02}}{G_{i j}^{0}}}\right|^{2} .
$$

The behavior of the transmission function depends qualitatively on whether the matrix elements of $G^{0}$ entering Eq. (35) are finite or not.

Consider the contribution of one of the nanotube lines $(l)$ to the noninteracting Green's function. Close to the resonance, $E=E_{\mu \mathbf{k}_{r}}, \mathbf{k}_{r}=\left(k_{\| r}, l\right)$, the denominators in Eqs. (12) can be approximated by the Taylor expansion

$$
z-E_{\mu \mathbf{k}} \approx-\frac{\partial E_{\mu \mathbf{k}_{r}}}{\partial k_{\|}}\left(k_{\|}-k_{\| r}\right)-\frac{1}{2} \frac{\partial E_{\mu \mathbf{k}_{r}}^{2}}{\partial k_{\|}^{2}}\left(k_{\|}-k_{\| r}\right)^{2}+i \eta,
$$

where derivatives are taken at the resonace point $k_{\|}=k_{\| r}$.

When the first derivative in Eq. (36) is different from zero the leading term of the real part of the denominator (36) is $\propto\left(k_{\|}-k_{\|_{r}}\right)$ which results in nondivergent sums (12) [and integrals in Eq. (15)], given the analytical behavior of the numerators in Eqs. (12). All matrix elements of $G^{0}$ are finite in this case and according to Eq. (35) the transmission is in the range $0<T<1$ for finite values of $\Delta$. For small and large values of $\Delta$ we have

$$
\begin{gathered}
T=4 \Delta^{2}\left|G_{i j}^{0}\right|^{2}, \quad \Delta G_{11}^{0} \ll 1, \\
T=\frac{4}{\Delta^{2}}\left|\frac{G_{i j}^{0}}{G_{i j}^{02}-G_{11}^{02}}\right|^{2}, \quad \Delta G_{11}^{0} \gg 1,
\end{gathered}
$$

i.e., the transmission vanishes at the extreme values of coupling strength to the leads. The same behavior as the function of coupling to the tip was predicted for the STM conductance. ${ }^{39}$

When the first derivative in the right-hand side of Eq. (36) is zero, the resonance point $\mathbf{k}_{r}$ coincides with one of the singular points $\mathbf{k}_{s}$ of the band structure. Then the expansion of the denominators begins with the term $\propto\left(k_{\|}-k_{\|_{s}}\right)^{2}$ which results in divergent sums (12). In this case $G_{11}^{0}$ is always infinite leading, in particular, to an infinite value of the density of states (Van Hove singularity). As for $G_{i j}^{0}$, it can have both finite and infinite values depending on the expression in the numerator of the second equation from Eq. (12). The transmission is fully determined by the third term in the denominator of Eq. (35). Even when $G_{i j}^{0}$ is infinite too, this term can be infinite if the factors $G_{i j}^{0}-G_{11}^{0}$ and $G_{i j}^{0}+G_{11}^{0}$ entering its numerator are both infinite. In this case the transmission is zero for any coupling $\Delta$. And vice versa, the third term in the denominator of Eq. (35) can be finite only if one of the above factors is finite. This means that the divergent parts of $G_{11}^{0}$ and $G_{i j}^{0}$ mutually cancel in one of the factors $G_{i j}^{0} \pm G_{11}^{0}$, and consequently the second term in the denominator of Eq. (35) becomes $i G_{11}^{0} / G_{i j}^{0}= \pm i$. The resulting transmission function is given in Table II. One can see that 
TABLE II. Transmission at the singularities of $G^{0}$.

\begin{tabular}{lcc}
\hline \hline$G_{i j}^{0}-G_{11}^{0}$ & $G_{i j}^{0}+G_{11}^{0}$ & $T$ \\
\hline infinite & infinite & 0 \\
finite & infinite & $\left|\frac{1}{i+\Delta\left(G_{i j}^{0}-G_{11}^{0}\right)}\right|^{2}$ \\
& & $\left|\frac{1}{-i+\Delta\left(G_{i j}^{0}+G_{11}^{0}\right)}\right|^{2}$ \\
\hline \hline
\end{tabular}

for finite values of one of the above factors the transmission goes to unity for small $\Delta$ and vanishes for large coupling strengths.

The divergent parts of $G_{11}^{0}$ and $G_{i j}^{0}$ (when $\mathbf{k}_{r}=\mathbf{k}_{s}$ ) arise from the zeroth term of the Taylor expansion at the $\mathbf{k}_{s}$ points of the corresponding numerators in Eqs. (12). These are 1 for $G_{11}^{0}, e^{i \mathbf{k}_{s} \Delta \mathbf{t}}$ for $G_{i j}^{0}$, when $i$ and $j$ correspond to carbon atoms of the same type, and $\sigma_{\mu} e^{i \mathbf{k}_{s} \Delta \mathbf{t}} f_{\mathbf{k}_{s}} /\left|f_{\mathbf{k}_{s}}\right|$ when $i$ and $j$ correspond to carbon atoms of different type. The divergent parts of $G_{11}^{0}$ and $G_{i j}^{0}$ will only coincide if their zeroth-order numerators will be equal in all points $\mathbf{k}_{s}$ corresponding to a given singularity, which implies the following relations for the numerators entering $G_{i j}^{0}$ :

$$
\begin{array}{r}
\operatorname{Re}\left\{\exp \left(i \mathbf{k}_{s} \Delta \mathbf{t}\right)\right\}=\cos \left(\mathbf{k}_{s} \Delta \mathbf{t}\right)= \pm 1 \quad \text { atoms same type, } \\
\operatorname{Re}\left\{\exp \left(i \mathbf{k}_{s} \Delta \mathbf{t}\right) \frac{f_{\mathbf{k}_{s}}}{\left|f_{\mathbf{k}_{s}}\right|}\right\}= \pm 1 \quad \text { atoms different type, }
\end{array}
$$

while the imaginary parts cancel for the pair of singular points $\mathbf{k}_{s},-\mathbf{k}_{s}$. As a rule, due to the inversion symmetry of the folded Brillouin zone [Fig. 1(b)], the fulfilment of Eq. (38) in one of the $\mathbf{k}_{s}$ points will extend automatically to another point of the same energy. This is certainly the case for all singularities in chiral nanotubes, whose Brillouin zones possess the inversion symmetry only. In achiral tubes, this is strictly correct only for singularities whose $\mathbf{k}_{s}$ points can be related by the inversion operation. However, there also exist in these tubes some $\mathbf{k}_{s}$ points related only by the mirror symmetry operations in the folded Brillouin zone [Figs. 2(a), 2(b)], which can in principle give different values on the RHS of the above equation for these points. Except for the latter cases Eq. (38) can be viewed as the necessary and sufficient condition for nonzero transmission at a given singularity $E=E_{\mu \mathbf{k}_{s}}$.

On the other hand the zero transmission at a singularity point can lead to two different types of behavior of $T(E)$ in the neighborhood of this point.

(1) $G_{i j}^{0}$ is divergent but the relations (38) do not hold at the singularity point. Then the divergent parts of $G_{11}^{0}$ and $G_{i j}^{0}$ do not compensate each other in either of the expressions $G_{i j}^{0} \pm G_{11}^{0}$. In this case a splitting of the peak of the transmission function in the point of the singularity emerges. The magnitude of this splitting is proportional to the noncompensated divergent part.
(2) $G_{i j}^{0}$ is not divergent at the singularity which implies the following relations for the corresponding zeroth-order numerators in Eq. (12):

$$
\begin{aligned}
\cos \left(\mathbf{k}_{s} \Delta \mathbf{t}\right) & =0 \quad \text { atoms same type, } \\
\operatorname{Re}\left\{\exp \left(i \mathbf{k}_{s} \Delta \mathbf{t}\right) \frac{f_{\mathbf{k}_{s}}}{\left|f_{\mathbf{k}_{s}}\right|}\right\} & =0 \quad \text { atoms different type, }
\end{aligned}
$$

as opposed to those in Eq. (38). In this case the transmission peak as a whole disappears from the region around the singularity, so one can speak of a missing peak. Again, one should stress that in achiral tubes the $\mathbf{k}_{s}$ points belonging to a given singularity, which cannot be related via the inversion relative to the $\Gamma$ point of the hexagonal Brillouin zone [Fig. $1(b)$, can give different values on the RHS of one of the above equations. Therefore in such cases the above equations do not represent the necessary condition for missing peaks.

\section{B. Zigzag nanotubes}

This is the most simple case of a single-wall nanotube. Since these tubes are characterized by the reduced indices $n^{\prime}=1, m^{\prime}=0$, only one single pole $\left(n^{\prime}+m^{\prime}=1\right)$ will contribute to the Green's function, Eqs. (B2) and (B3). This pole corresponds to one of the two zeroes of the second order polynomial $p(w)$ in Eq. (28) (Appendix B).

The real part of $k_{\|}$of the contributing pole of each nanotube line $l$

$$
-n / 2<l \leqslant n / 2 \text {, }
$$

is found between two extrema 0 and $\pm 2 \pi / \sqrt{3} a$, confining half of the corresponding band [Fig. 3(a)]. The energies of these extrema, written for $(n,-n)$ tubes $\left[\gamma_{2}=\gamma_{3}\right.$, see Fig. $1(\mathrm{a})]$, in each band $\mu$ of the nanotube line $l$,

$$
\begin{gathered}
E_{l}^{1}=\sigma_{\mu}\left|\gamma_{1}-2 \gamma_{2} \cos \frac{\pi l}{n}\right|, \quad k_{\|}=\frac{2 \pi}{\sqrt{3} a}, \\
E_{l}^{2}=\sigma_{\mu}\left|\gamma_{1}+2 \gamma_{2} \cos \frac{\pi l}{n}\right|, \quad k_{\|}=0,
\end{gathered}
$$

define the positions of Van Hove singularities in the DOS of zigzag nanotubes [Fig. 3(c)]. One can see that all extrema are situated on the planes of symmetry. In this case the real part of $k_{\|}$of the contributing pole will remain constant (equal to its value at the singularity) for all energies outside the corresponding band, a situation similar to the case of a monoatomic chain.

The curvature of the tube leads to the relation between transfer parameters ${ }^{52,53}\left|\gamma_{1}\right|>\left|\gamma_{2}\right|$ for $(n,-n)$ zigzag tubes, due to which primary metallic nanotubes become semiconducting, with the gap $2 \Delta \gamma, \Delta \gamma=\left|\gamma_{1}-\gamma_{2}\right|$, opening along the line $l=n / 3$ [Fig. 3(b)]. In the middle of this gap $(E$ $=0$ ), the transmission will be zero in the case when the leads are attached at atoms of the same type, since both $G_{11}^{0}$ and $G_{i j}^{0}$ vanish at this energy [see Eqs. (12) and (13)]. This arises from the exact cancellation of the contributions of the 

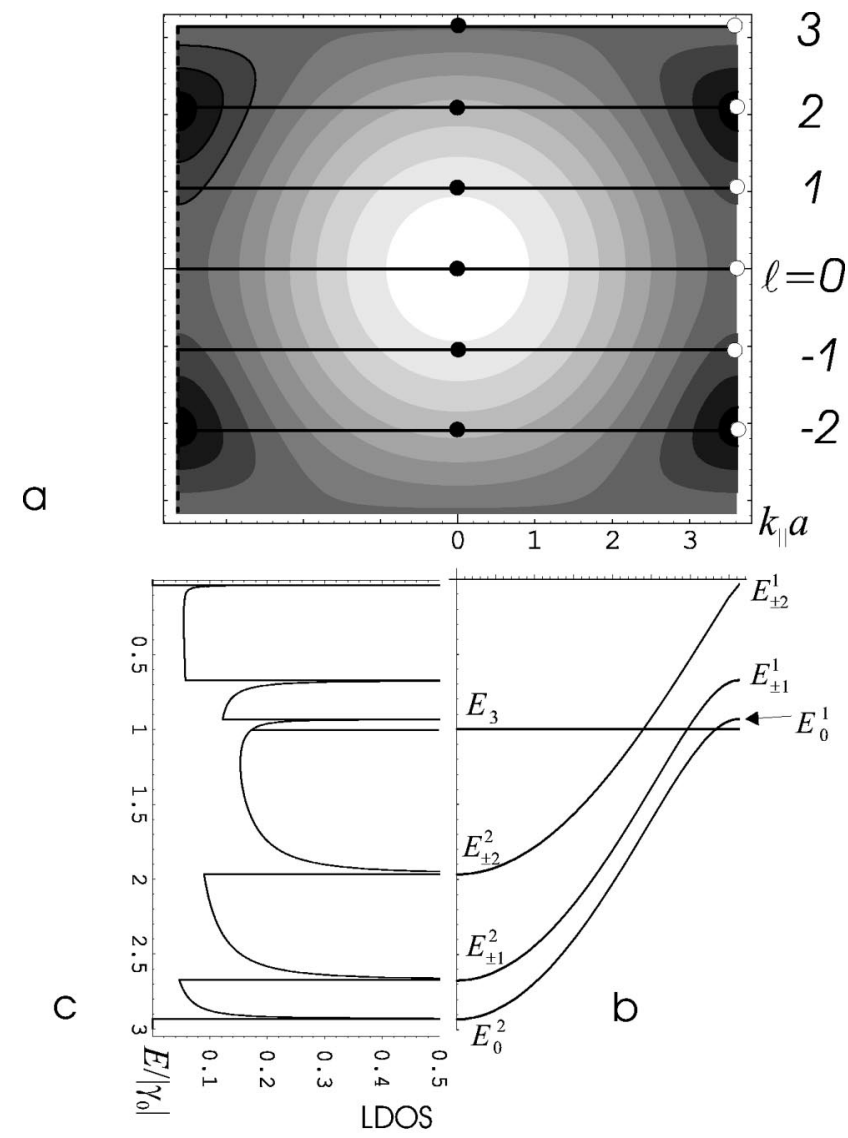

FIG. 3. Rectangular Brillouin zone (a), energy dispersion along the nanotube lines (b) and density of states per carbon atom (c) of the $(6,-6)$ zigzag nanotube. Solid straight lines in (a) stand for nanotube lines (numbered in italics), while open and filled circles show the position of singularities. The contour plot in (a) shows the lowest $\pi$ band of graphite in the rectangular Brillouin zone (dark regions indicate higher energies). The energy labels in (b) show the position of the corresponding singularities. Only half of the energy spectrum is shown.

two bands belonging to the same nanotube line which, in turn, is the result of the mirror symmetry of the bands with respect to $E=0$. This feature is actually an artifact of our orthogonal tight-binding model ${ }^{54-57}$ therefore a shift of this zero transmission point away from $E=0$ is expected when the effects of band asymmetry are included. For small deviations $\Delta E$ from this symmetry point, the transmission will rise as $\Delta E^{2}$ according to Eqs. (33) and (34). As for transmission between carbon atoms of different type, the second relation from Eq. (12) suggests that the contributions from mirror bands will just add to each other in contrast to the previous case.

\section{Transmission at singularities}

Let us analyze the transmission at singularity points given by Eqs. (41) first. The corresponding singularities in $\mathbf{k}$ space for $|l|<n / 2$ are defined by $k_{\|}$in Eqs. (41) and $k_{\perp}$ :

$$
k_{\perp}=\frac{2 \pi l}{a n} .
$$
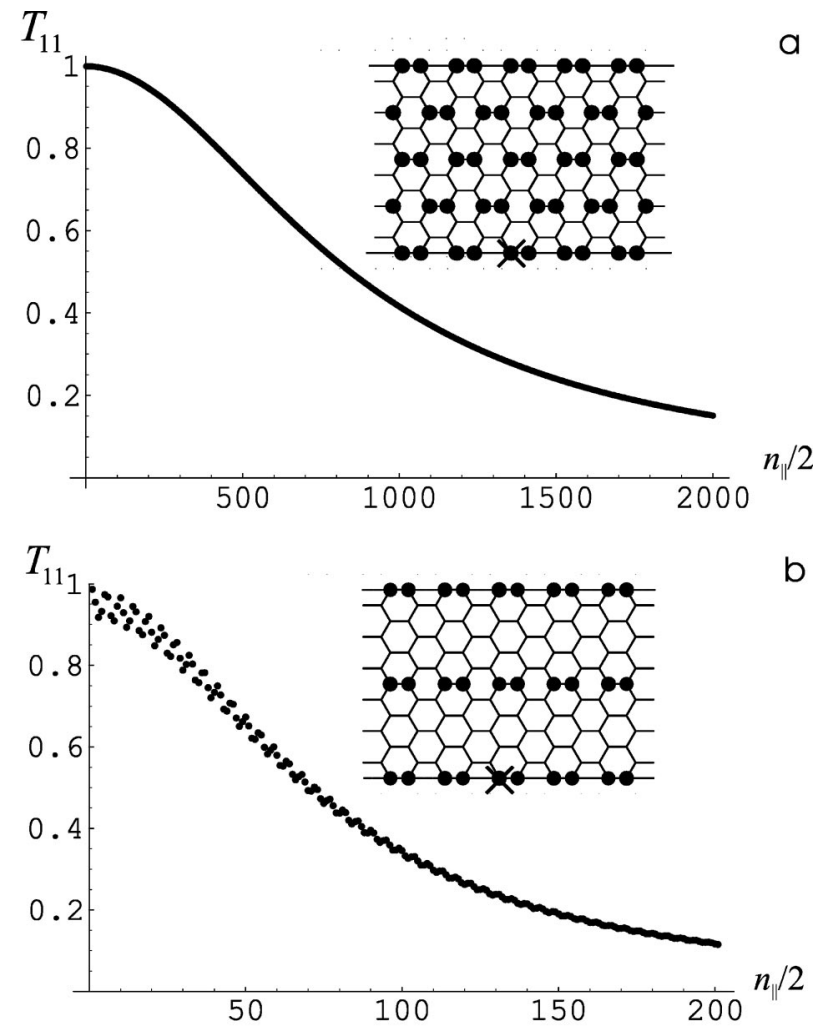

FIG. 4. Distance dependence in the axial direction of the nonzero transmission at the singularity $E_{ \pm 2}^{1}$ lying on the Fermi level (a) and at the singularity $E_{ \pm 1}^{1}$ (b) of the $(6,-6)$ nanotube (Fig. 3). The insets show the maps of possible positions of monoatomic contacts on the surface of the nanotube's strip; the cross shows the position of the first tip and one of the black circles the position of the second tip. The coupling strength to the leads is $\Delta=0.1\left|\gamma_{0}\right|$.

It is easily seen from Figs. 2(a) and 3(a) that for all singularities $(l \neq 0)$ the corresponding pairs of $\mathbf{k}_{s}$ points can be related via the inversion operation relative to the center of the hexagonal Brillouin zone. Using Eqs. (41), (42), and (22) [in $(n,-n)$ tubes we have $n_{\|}=-n_{x}, n_{\perp}=n_{y}$ ] and taking into account that $f_{\mathbf{k}_{s}}$ is real in all singularity points, the condition for nonzero transmission at singularities, Eq. (38), in arbitrary zigzag nanotubes becomes

$$
\frac{l}{n} n_{\perp}=\text { integer. }
$$

The inset of Fig. 4(a) shows the map of nonzero transmission at the singularity $E_{ \pm 2}^{1}$ coinciding with the Fermi level of undoped $(6,-6)$ nanotube (Fig. 3). Other relative positions of the two carbon atoms, not obeying Eq. (43), correspond to splitting or completely missing peaks of transmission at a given singularity. Substituting Eqs. (41), (42), and (22) into Eq. (39) we obtain the following condition for missing transmission peaks in arbitrary zigzag nanotubes:

$$
2 \frac{l}{n} n_{\perp}=\text { odd integer. }
$$




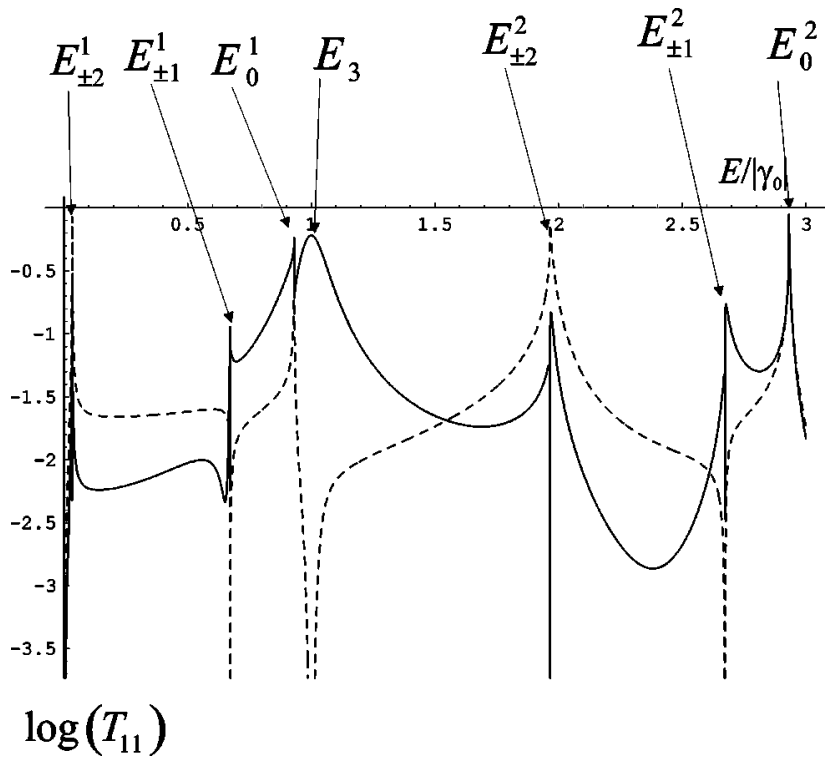

FIG. 5. Energy profile of the transmission through a $(6,-6)$ nanotube (logarithmic scale) for two relative positions of the tips contacting atoms of the same type: (a) $n_{\|}=0, n_{\perp}=4$ (full line); (b) $n_{\|}=1, n_{\perp}=3$ (dashed line). The coupling strength to the leads is $\Delta=0.5\left|\gamma_{0}\right|$.

This condition can only be fulfilled for even $n$. It follows from the above equations that for the two singularities at the $l=0$ nanotube line, corresponding to the $\mathbf{k}_{s}$ singular points $\Gamma$ and $M$ respectively [Fig. 1(b)], the transmission is always different from zero.

Figure 5 shows how the change of transmission at singularities as function of relative positions of the tips, described by Eqs. (43) and (44), modifies the transmission profile in a $(6,-6)$ tube. Going from $n_{\|}=0, n_{\perp}=4$ to $n_{\|}=1, n_{\perp}=3$, the peaks at $E_{ \pm 2}^{1,2}$ turn from splitted into normal ones, the splitted peaks at $E_{ \pm 1}^{1,2}$ become missing and the strong trans-

$$
\tilde{z}=\left\{\begin{array}{l}
z \\
\left(\gamma_{1}+2 \gamma_{2} \cos \frac{\pi l}{n} w_{ \pm, l}\right) \\
\left(\gamma_{1}+2 \gamma_{2} \cos \frac{\pi l}{n} w_{ \pm, l}^{-1}\right.
\end{array}\right)
$$

In these equations, the sign in the subscript of $w$, for each nanotube line, is chosen according to the rule given in Appendix B. For instance, in the region close to $E_{F}, E<\left|\gamma_{1}\right|$, only $w_{+}$enter the above equations. The first term on the RHS of Eq. (46) incorporates the two contributions from the nanotube line $l^{\prime}$ becoming divergent at a singularity point. Taking the limit $z \rightarrow E_{l^{\prime}}^{1,2}$ we obtain for this term mission peak at $E_{3}$ also disappears. At the same time, the peaks at $E_{0}^{1,2}$ are always normal ones according to Eq. (43).

To find the distance dependence of the transmission at singularities one should evaluate $G_{11}^{0} \pm G_{i j}^{0}$ entering the expressions for the transmission in Table II. The sign in this combination is defined by the sign of the two factors depending on $n_{\perp}$ and $n_{\|}$[see Eqs. (B2) and (B3)], in the contribution corresponding to the nanotube line $l^{\prime}$ containing the given singularity $E_{l^{\prime}}^{1,2}$. One finds that the Green's functions enter the expression for a nonzero transmission at a singularity always in the form $G_{11}^{0}-\xi G_{i j}^{0}$, with $\xi$ defined as

$$
\xi=(-1)^{\frac{l^{\prime}}{n}} n_{\perp}( \pm 1)^{n} \| \begin{array}{ll}
1 & \text { atoms same type, } \\
\operatorname{sgn}(\gamma E) & \text { atoms different type }
\end{array}
$$

where the two signs of the unity correspond to $E_{l^{\prime}}^{2}$ and $E_{l^{\prime}}^{1}$, respectively. Using Eqs. (B2) and (B3) we obtain

$$
\begin{aligned}
G_{11}^{0}-\xi G_{i j}^{0}= & -\frac{1}{n}\left(\left(2-\delta_{l^{\prime}, 0}\right) z \frac{1-\left|w_{ \pm, l^{\prime}}\right|^{\mid n \|} \mid}{ \pm D_{l^{\prime}}}\right. \\
& \left.+\sum_{l\left(\neq \pm l^{\prime}\right)} \frac{z-\xi \tilde{z} \cos \left(\pi \frac{l}{n} n_{\perp}\right) w_{ \pm, l}^{\left|n_{n}\right|}}{ \pm D_{l}}\right),
\end{aligned}
$$

where $\left|w_{ \pm, l^{\prime}}\right|$ is understood as $-w_{ \pm, l^{\prime}}$ for singularities of the first type and $w_{ \pm, l}$ for singularities of the second type. As in Eqs. (B2) and (B3), the sign of $D$ corresponds to the subscript of $w$. In addition, an explicit dependence of $D$ and $w$ on the nanotube line's index was introduced as an additional subscript. In Eq. (46) the following notation is introduced:

atoms same type,

atoms different type, $\quad n_{\|} \geqslant 0$,

atoms different type, $\quad n_{\|}<0$.

$$
\pm\left(2-\delta_{l^{\prime}, 0}\right) E_{l^{\prime}}^{1,2} \frac{\left|n_{\|}\right|}{4 \gamma_{1} \gamma_{2} \cos \left(\frac{\pi l^{\prime}}{n}\right)},
$$

where the two signs in front correspond to the singularity of the first and the second kind [Eq. (41)], respectively. We can 
see that although the diverging parts of $G_{11}^{0}$ and $G_{i j}^{0}$ have mutually cancelled in this term, the remaining part shows a linear increase with the distance between contacted carbon atoms and therefore can become very large. At the same time the other contributions $l \neq \pm l^{\prime}$ in Eq. (46) can either oscillate or vanish with increasing the distance between tips. As a result the term (48) becomes dominant and defines the behavior of the transmission at large distances. In particular it shows that the transmission will decrease with distance as $n_{\|}^{-2}$. The same behavior of the transmission was found for long monoatomic chains at the edges of their bands, where Van Hove singularities arise. ${ }^{39}$ It follows from Eqs. (46) and (48) that the transmission in this asymptotic region should be higher at singularities which are closer to the Fermi level, and it also increases with the radius of the tube. Figure 4(a) shows the transmission at the singularity $E_{ \pm 2}^{1}$, pinned at the Fermi level in the $(6,-6)$ nanotube as function of the axial distance between contacted carbon atoms. The main contribution to the transmission at this energy comes from the term (48) while the contributions from other lines in Eq. (46) decay exponentially with $\left|n_{\|}\right|$since their bands do not overlap with the given singularity (Fig. 3). As for the distance dependence in the circumferential direction, it will not show up in the limit of large distances, according to Eq. (48).

The nanotube line $l=n / 2$ ( $n$ even), in contrast to the previous ones, contains completely flat bands $E= \pm\left|\gamma_{1}\right|$, Fig. 3(b), which correspond to the lines of singularities of the Green's function. This means that our analysis of the transmission, developed in the previous section for point singularities, does not apply here. Nevertheless the expressions for the transmission function given in Table II still hold since they were derived on general grounds. Using Eq. (B4) for the diverging part of the Green's function at $E= \pm\left|\gamma_{1}\right|$ we obtain that the transmission can only be different from zero between carbon atoms with axial spacing $n_{\|}=0$. In contrast to the case of transmission at the first singularity $E_{ \pm 2}^{1}$, now all lines give nonzero contributions to the transmission [see Fig. 3(b)]. Among the lines $l \neq n / 2$ the main contribution to Eq. (46) comes from $l=0$ since its singularity $E_{0}^{1}$ lies at a distance of $\Delta \gamma$ from $\gamma_{1}$ [Eq. (41)]. Taking into account these two leading contributions we obtain for the transmission function at $E= \pm\left|\gamma_{1}\right|$ :

$$
T_{11}= \begin{cases}1, & n_{\perp} / 2 \text { even }, \\ \frac{1}{\left(1+\frac{\Delta}{2 n \sqrt{2 \Delta \gamma\left|\gamma_{0}\right|}}\right)^{2},} & n_{\perp} / 2 \text { odd }\end{cases}
$$

for atoms of the same type and

$$
T_{12}= \begin{cases}\frac{1}{1+\left(\frac{\Delta}{2 n \gamma_{0}}\right)^{2},} & n_{\perp} / 2 \text { odd }, \\ \frac{1}{\left(1+\frac{\Delta}{\left.2 n \sqrt{2 \Delta \gamma\left|\gamma_{0}\right|}\right)^{2}+\left(\frac{\Delta}{2 n \gamma_{0}}\right)^{2}},\right.} & n_{\perp} / 2 \text { even }\end{cases}
$$

for atoms of different type. For all other relative positions of contacted carbon atoms we have $G_{i j}^{0}=0$ while $G_{11}^{0}$ still divergent, according to Eq. (B4), which means that zero transmission at these positions is always associated with a missing transmission peak at $E= \pm\left|\gamma_{1}\right|$ (Fig. 5). As in the case of point singularities, there is no distance dependence of the transmission in the circumferential direction, which is thus nondecaying. On the whole the transmission at $E= \pm\left|\gamma_{1}\right|$ goes only via individual ladders in this direction which thus look as being effectively decoupled.

\section{Transmission in the gap region}

Consider now the transmission at energies away from singularity points. The region of practical interest lies around the Fermi energy. In primary metallic tubes with indices $n$ multiple to 3 , the transmission at energies close to the Fermi level is mainly determined by the contributions from the nanotube lines $l= \pm n / 3$ crossing the Fermi points $K_{1}$ and $K_{2}$ of the graphene's Brillouin zone [Fig. 3(a)]. The corresponding expressions for the transmission function in this energy region are derived in Appendix $\mathrm{C}$.

In the middle of the energy gap, $E=0$, the transmission $T_{i j}$ between carbon atoms $i, j$ vanishes for atoms of the same type due to symmetry reasons mentioned above, while it is nonzero for atoms of different type. With Eqs. (33), (34), and (C11) we obtain

$$
T_{12}= \begin{cases}\frac{16 \Delta^{2}}{n^{2} \gamma_{1}^{2}} \cos ^{2}\left(\frac{\pi}{3} n_{\perp}\right) e^{-2 \ln \left(\frac{\gamma_{1}}{\gamma_{2}}\right)\left|n_{\|}\right|} & \\ \left(1+\frac{4 \Delta^{2}}{n^{2} \gamma_{1}^{2}} \cos ^{2}\left(\frac{\pi}{3} n_{\perp}\right) e^{\left.-2 \ln \left(\frac{\gamma_{1}}{\gamma_{2}}\right)\left|n_{\|}\right|\right)^{2}},\right. & n_{\|} \geqslant 0, \\ 0, & n_{\|}<0 .\end{cases}
$$

The corresponding map of transmission is shown in Fig. 6. We meet an interesting phenomenon here. With respect to the tube's axis, the atoms of the type 1 will mediate appreciable transmission only via atoms on their left, while the atoms of type 2 -only via atoms on their right.

The contributions of other nanotube lines, $l \neq \pm n / 3$, for $E=0$ are given by the corresponding poles $w_{+, l}$, Eq. (C1) $\left(\left|\gamma_{1}\right|>\left|\gamma_{2}\right|\right)$ which, inserted into Eq. (B3), give the following additions to $G^{0}$ in Eq. (C11):

$$
\begin{aligned}
G^{0}(\Delta \mathbf{t}, 12 ; 0)= & -\frac{1}{n \gamma_{1}}(-1)^{n \|}\left[(-1)^{n_{\perp} / 2} \delta_{n_{\|}, 0}\right. \\
& \left.+2 \sum_{1 / 3<l / n<1 / 2} \cos \left(\frac{\pi l}{n} n_{\perp}\right)\left(2 \frac{\gamma_{2}}{\gamma_{1}} \cos \frac{\pi l}{n}\right)^{\mid n \|}\right], \\
& n_{\|} \geqslant 0,
\end{aligned}
$$




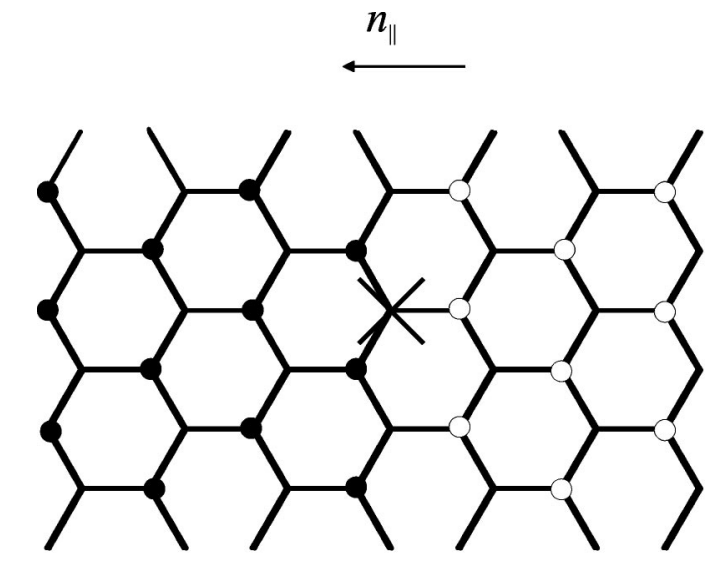

FIG. 6. Map of nonzero transmission at $E=0$ in a primary metallic zigzag nanotube. Filled and empty circles correspond to high and low transmission [see Eqs. (51)]. The first contacted atom is indicated by a cross.

$$
\begin{aligned}
G^{0}(\Delta \mathbf{t}, 12 ; 0)= & \frac{1}{n \gamma_{1}}(-1)^{n \|}\left[\left(\frac{\gamma_{1}}{2 \gamma_{2}}\right)^{\left|n_{\|}\right|}\right. \\
& \left.+2 \sum_{0<l / n<1 / 3} \cos \left(\frac{\pi l}{n} n_{\perp}\right)\left(\frac{\gamma_{1}}{2 \gamma_{2}} \frac{1}{\cos \frac{\pi l}{n}}\right)^{\left|n_{\|}\right|}\right], \\
& n_{\|}<0 .
\end{aligned}
$$

Interestingly, the propagation in the two directions of $n_{\|}$is sustained by different nanotube lines. The contributions of other lines vanish at $E=0$. Although at small $n_{\|}$the terms in the above equations are of the same order of magnitude as $G^{0}\left(n_{\|} \geqslant 0\right)$ in Eq. (C11), they decay much faster with the axial distance between the leads. This becomes clear when we examine the deviation of the corresponding $w_{+, l}$ from unity. For $l= \pm n / 3$ this deviation is $\approx \Delta \gamma /\left|\gamma_{0}\right|=C / n^{2}$ (see Ref. 20), with $C=\pi^{2} / 8$ for zigzag tubes. ${ }^{58}$ At the same time this deviation is much larger for other nanotube lines, even for the closest ones to the "metallic" line. For instance, the deviation of $w_{+, l^{\prime}}, l^{\prime}=n / 3-1$, from unity is $\sqrt{3} \pi / n$. This means that the contribution from "nonmetallic" nanotube lines, Eq. (52), is small already for $n_{\|} \approx n$. Therefore the strong anisotropy of the transmission, described in Eq. (51), persists at distances large enough between the leads. As Eq. (C13) shows, the sign of this anisotropy changes for the opposite relationship between transfer parameters $\left|\gamma_{1}\right|$ $<\left|\gamma_{2}\right|$. This, however, does not affect the additional contributions from "nonmetallic" lines in Eq. (52). Finally, in the limit $\gamma_{1}=\gamma_{2}$ the contributions $l= \pm n / 3$ are obtained from the band solutions in Eqs. (C7) and (C8) for $\Delta \gamma=0$ at $E$ $=0$ :

$$
G^{0}(\Delta \mathbf{t}, 12 ; 0)=\mp \frac{1}{n \gamma_{0}} \cos \left(\frac{\pi}{3} n_{\perp}\right)(-1)^{n} \|,
$$

where the two signs correspond to $n_{\|} \geqslant 0$ and $n_{\|}<0$. Comparing to the gap solutions $(\mathrm{C} 11)$ and $(\mathrm{C} 13)$ we conclude that the propagation amplitude from the "metallic" lines is now equally shared between the two directions, the same for the transmission.
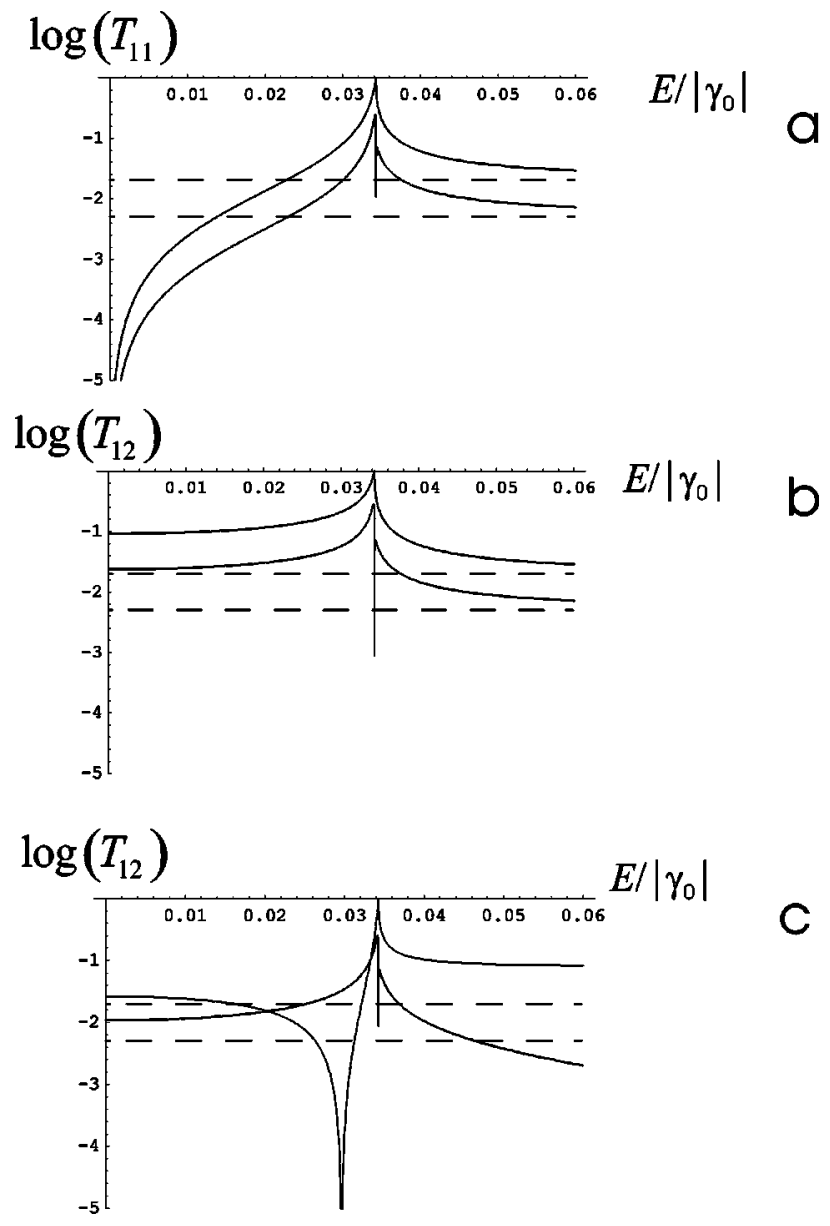

FIG. 7. Energy dependence of the transmission close to the Fermi level for a $(6,-6)$ nanotube $\left(\Delta \gamma=0.0343\left|\gamma_{0}\right|\right)$ between atoms of the same type $n_{\|}=2$ (a) and different type for $n_{\|}=2$ (b) and $n_{\|}=-2$ (c). Continuous lines show the full transmission function and the dashed lines the "metallic" contribution only in the absence of curvature effects [Eq. (54)]. The upper and lower curve (seen from the high energy side) correspond to $n_{\perp}=0$ and $n_{\perp}=2$, respectively. The coupling strength to the leads is $\Delta=0.5\left|\gamma_{0}\right|$.

At $E=0$ the transmission has the fastest exponential decay as function of axial distance (for $n_{\|}$large enough), defined by the coefficient $2 \ln \left(\gamma_{1} / \gamma_{2}\right) \approx 2 \Delta \gamma /\left|\gamma_{0}\right|$ in the exponent, Eq. (51). However, this decay is still much slower than in monoatomic chains which, for the same relative deviation from the band edge $\Delta \gamma /\left|\gamma_{0}\right|$, can be shown to be $2\left|\Delta \gamma / \gamma_{0}\right|^{1 / 2}$. Therefore, in the gap region, zigzag nanotubes are expected to be much better electronic guides than single band chains. Beyond $E=0$ the transmission will increase while its decay with axial distance will become less steep according to Eq. (C5), merging finally with the value at the first singularity $E= \pm \Delta \gamma[$ Fig. $4(\mathrm{a})]$ for both types of contacted atoms.

The energy behavior of the transmission close to the Fermi level is shown in Fig. 7 by continuous lines. A new feature arising in the gap region is the antiresonance of the transmission $(T=0)$ between atoms 1 and 2 in the negative axial direction $n_{\|}<0$ at $E \approx 0.03\left|\gamma_{0}\right|[$ Fig. 7(c)]. As in the case of atoms of the same type at $E=0$ [Fig. 7(a)], this antiresonance arises from the zero value of the corresponding 
Green's function. However, unlike the former case, the antiresonance in Fig. 7(c) is not due to symmetry effects in the interference of different contributions but is rather conventional..$^{59}$ The reason for such an antiresonance is the sign difference which individual contributions to the Green's function in Eqs. (B2) and (B3) can have as function of $n_{\perp}$, given that all these contributions are real in the gap region. Apart from $\cos \left[(\pi l / n) n_{\perp}\right]$, all other factors entering $G^{0}$ have constant signs. Thus, for $\gamma_{i}<0$, the expression in the last bracket of Eq. (B3) corresponding to the "metallic" nanotube lines is negative for both $n_{\|} \geqslant 0$ and $n_{\|}<0$. Other nanotube lines give positive values for this expression for $n_{\|}<0$ and negative values for $n_{\|} \geqslant 0$. This means, in particular, that for $n_{\perp}=0$ the antiresonance condition can only be met for atoms of different type for $n_{\|}<0$, since only in this case can we have contributions of different sign. The condition for antiresonances is sensible to the relative orientation of the leads due to a strong dependence of individual contributions to $G^{0}$ on $n_{\|}$and $n_{\perp}$.

\section{Transmission in the band region}

In the band region $|E|>\Delta \gamma$, the transmission will decrease with the deviation of the energy from the first singularity, according to Eqs. (C8) and (C9). Concomitantly, the difference between the "metallic" contributions, corresponding to the transmission through different pairs of carbon atoms [specified in Eq. (C8)] with the same relative shifts $\left(n_{\|}, n_{\perp}\right)$, will diminish. In the limit $\Delta \gamma^{2} \ll E^{2}$, which also corresponds to the neglect of the curvature effects in the electronic structure of these tubes, the transmission is described by

$$
T=\frac{\frac{4 \Delta^{2}}{n^{2} \gamma_{0}^{2}} \frac{1}{\left[1-\left(E / 2 \gamma_{0}\right)^{2}\right]} \cos ^{2}\left(\frac{\pi}{3} n_{\perp}\right)}{\left|\left(1+\frac{\Delta}{n\left|\gamma_{0}\right|} \frac{1}{\sqrt{1-\left(E / 2 \gamma_{0}\right)^{2}}}\right)^{2} \mp \frac{\Delta^{2}}{n^{2} \gamma_{0}^{2}} \frac{1}{1-\left(E / 2 \gamma_{0}\right)^{2}} \cos ^{2}\left(\frac{\pi}{3} n_{\perp}\right) e^{2 i\left(E /\left|\gamma_{0}\right|\right)\left|n_{\|}\right|}\right|^{2}},
$$

where the upper and the lower sign in the denominator correspond to contacted atoms of the same and different type, respectively. We can see that in this limit (approximation) the transmission is almost energy independent for small $E$. The corresponding transmission functions are shown in Fig. 8 by thin dashed (a) and dash-dotted (b) lines. With respect to them the full transmission displays different behaviors for different kinds of pairs of contacted carbon atoms. In the case of atoms of the same type the full transmission approaches asymptotically the "metallic" contribution for $\Delta \gamma$ $=0[$ Fig. $7(\mathrm{a})]$. This is due to the fact that the contributions from other nanotube lines still remain unimportant for small deviations $E /\left|\gamma_{0}\right|$ from the center of the band structure ( $E$ $=0)$, where they vanish exactly. The same asymptotic behavior is observed for transmission between atoms of type 1 and 2 for $n_{\|}>0$ [Fig. 7(b)]. In this case the lack of the effect from "nonmetallic" nanotube lines is specific to the $(6$, $-6)$ tube. Indeed, as the first equation from Eq. (52) shows, the contributions from the lines $1 / 3<|l / n| \leqslant 1 / 2$, which exist already at $E=0$, are absent in this tube because of its small diameter. At the same time the contributions from other lines $0 \leqslant|l / n|<1 / 3$ will still remain negligible for small values of $E /\left|\gamma_{0}\right|$, similarly to the case of contacted atoms of the same type. On the other hand the transmission between different atoms in the opposite direction, $n_{\|}<0$, in the same tube does not show asymptotic behavior [Fig. 7(c)] since the "nonmetallic" contributions already show up via the nanotube lines $|l / n|=0,1 / 6$ [see the second expression in Eq. (52)]. Figure 8(a) shows the transmission for $n_{\|}=-2, n_{\perp}=2$ in a broader energy range together with the individual contribution from $l / n= \pm 1 / 3$. The depletion of the total transmission (solid line) with respect to a pure "metallic" contribution (thin line) for $0.03<E /\left|\gamma_{0}\right|<0.5$ is due to the opposite sign of "nonmetallic" contribution in the Green's function of this tube for $n_{\perp}$ not a multiple to three. On the other hand, since the "metallic" contribution itself is complex in the band region, Eqs. (C7) and (C8), the antiresonance condition cannot be met. Increasing $\left|n_{\|}\right|$will diminish the "nonmetallic" contributions only [Eqs. (52)], while the transmission function will approach asymptotically the "metallic" contribution in the band region [Fig. 8(b)].

Equation (C9) shows that in the band region the transmission can only oscillate as a function of the axial distance between contacted atoms. The oscillatory behavior is determined by the last term in the denominator of this expression via the factor $\exp \left(i \beta \mid n_{\|}\right)$, where $\beta \approx 2 \operatorname{sgn}(E) \sqrt{2 \Delta \gamma \delta E} /\left|\gamma_{0}\right|$ and $\delta E$ is a small deviation from the band edge (first singularity). Once again, one should stress the marked difference from the case of monoatomic chains where, for the same energy deviation from the band edge relative to the transfer parameter $\delta E /\left|\gamma_{0}\right|$, the corresponding coefficient $\beta \approx$ $-2 \operatorname{sgn}(E) \sqrt{\delta E /\left|\gamma_{0}\right|}$ is much larger and the oscillations of the transmission are correspondingly much faster. Close to the first singularity $|E|=\Delta \gamma$, the oscillating term in the denominator of Eq. (C9) becomes large leading to appreciable amplitudes of oscillations of the transmission with the axial distance between the leads. Going deeper into the band, the transmission will decrease following roughly the energy dependence of the factor $h_{i j}^{2}$ in the numerator of Eq. (C9). The weight of the oscillating term in the denominator will decrease at the same extent thus reducing the amplitude of oscillations of the transmission. 
According to Eq. (54) the transmission will remain almost unchanged in the energy region $\Delta \gamma^{2} \ll E^{2} \ll \gamma_{0}^{2}$, being of the order of $4 \Delta^{2} / n^{2} \gamma_{0}^{2}$. However, this equation applies only when the effect of the second singularity $E_{l^{\prime}}^{1}, l^{\prime}=n / 3-1$ can be neglected. Actually this is still the case for transmission energies quite close to $E_{l^{\prime}}^{1}$. At the point of the second singularity the transmission shows a decaying-oscillating behavior as a function of the axial distance between contacted atoms [Fig. 4(b)]. This can be understood by evaluating the appropriate combination of the Green's functions, entering the expression for the transmission in Table II, at this singularity. Using Eqs. (46)-(48) we obtain

$$
\begin{aligned}
& G_{11}^{0}-\xi G_{i j}^{0}=-\frac{1}{n}\left(E_{l^{\prime}}^{1} \frac{\left|n_{\|}\right|}{2 \gamma_{0}^{2} \cos \left(\frac{\pi l^{\prime}}{n}\right)}+i \frac{1-\xi \frac{\tilde{z}}{E_{l^{\prime}}^{1}} \cos \left(\frac{\pi}{3} n_{\perp}\right)(-1)^{n} \| e^{i\left(E_{l^{\prime}}^{1}|| \gamma_{0} \mid\right)\left|n_{\|}\right|}}{\left|\gamma_{0}\right| \sqrt{1-\left(E_{l^{\prime}}^{1} / 2 \gamma_{0}\right)^{2}}}\right) \text {, } \\
& \tilde{z}= \begin{cases}E_{l^{\prime}}^{1} \approx \sigma_{\mu}\left|\gamma_{0}\right|\left(1-\cos \frac{\pi}{n}-\sqrt{3} \sin \frac{\pi}{n}\right), & \text { atoms same type, } \\
\left|\gamma_{0}\right|\left(1-e^{ \pm i\left(E_{l^{\prime}}{ }^{\prime}\left|\gamma_{0}\right|\right)}\right), & \text { atoms different type, }\end{cases}
\end{aligned}
$$

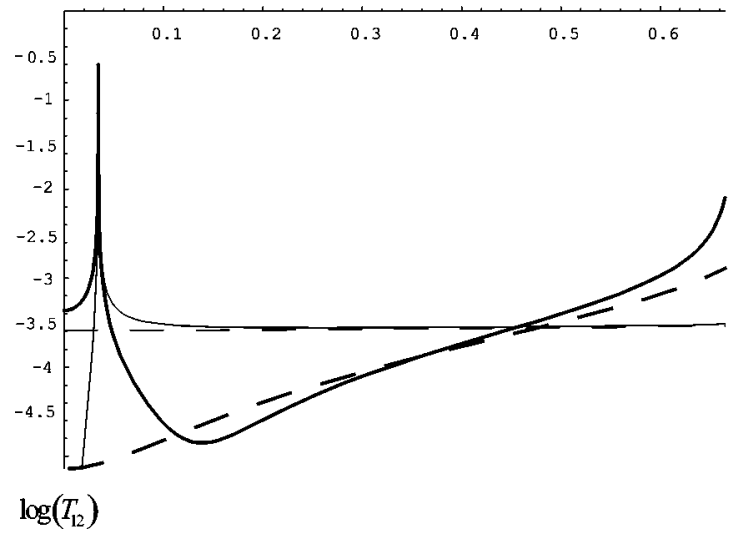

$E /\left|\gamma_{0}\right|$

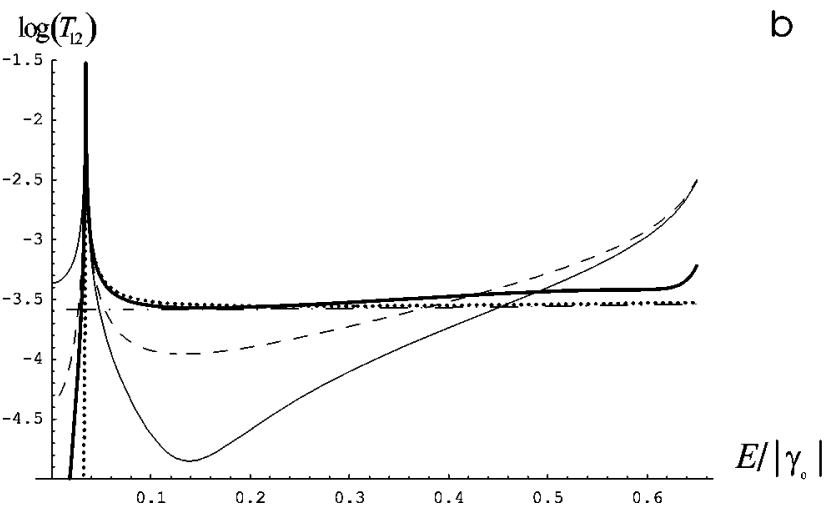

FIG. 8. Energy dependence of the transmission in the $(6,-6)$ nanotube $\left(\Delta \gamma=0.0343\left|\gamma_{0}\right|, \Delta=0.1\left|\gamma_{0}\right|\right)$ between atoms of different type for $n_{\perp}=2$ : (a) $n_{\|}=-2$; full transmission (solid lines) and the "metallic" contribution only (thin lines), with (continuous) and without (dashed) curvature effects; (b) full transmission at $n_{\|}$ $=-2$ (thin solid), $n_{\|}=-4$ (thin dashed), $n_{\|}=-8$ (solid) and $n_{\|}$ $=-200$ (dotted); the dash-dotted line corresponds to the "metallic" contribution without curvature effects. where $\xi$ is defined in Eq. (45) and the two signs in the exponent of the last expression correspond to $n_{\|} \geqslant 0$ and $n_{\|}$ $<0$. In evaluating these expressions, the terms corresponding to "metallic" lines $l= \pm n / 3$ in the summation in Eq. (46) have been included, since these are the only additional lines whose bands are intersected by the energy $E_{l^{\prime}}^{1}$ [Fig. 3(b)]. At the same time the curvature effects (terms $\sim \Delta \gamma$ ) are unimportant at this energy point. For low values of $n_{\|}$both terms in RHS of the first equation in Eq. (55) are of the same order. As a result the amplitude of oscillations of the transmission determined by the second term is large. The oscillation behavior is only due to the exponential entering this term, while the factor $(-1)^{n} \|$ is a constant since $n_{\|}$has the parity of $n_{\perp}$. Increasing the axial distance between contacted atoms results in the increase of the first term and in the $1 / n_{\|}^{2}$ decrease of transmission, according to the general rule in Eq. (48). This decrease is much steeper as compared to the transmission at the first singularity [Fig. 4(a)], which is explained by a larger factor $E_{l^{\prime}}^{1}$ in the first term. At the same time the relative weight of the oscillating term on the RHS of the first expression in Eq. (55) will diminish with increasing $n_{\|}$, the same for the amplitude of oscillation of the transmission function, clearly seen in Fig. 4(b). All other nanotube lines $l$, not included in the above treatment, will have $\left|w_{+, l}\right|<1$ at $E=E_{l^{\prime}}$ [Eqs. (B1) and (C1)]. Therefore the corresponding contributions in Eq. (46) are only important at small values of $n_{\|}$and will decay exponentially with increasing the axial distance between contacted atoms. Note the complete analogy in the behavior of these additional contributions to the case of the transmission energy near the Fermi level, described by Eqs. (52).

The obtained results are general not only for primary metallic but also for primary semiconducting zigzag nanotubes. The only difference is that the band gap is much larger for the latter. 


\section{Armchair nanotubes}

These tubes are next in complexity since they are characterized by the reduced indices $n^{\prime}=1, m^{\prime}=1$ and therefore two poles $\left(n^{\prime}+m^{\prime}=2\right)$ will contribute to the Green's function, Eqs. (B6) and (B7). These poles correspond to two of the four zeroes, Eq. (B5), of the fourth order polynomial $p(w)$.

The nanotube lines contained in the rectangular Brillouin zone of armchair tubes [Fig. 2(b)] are described by the same indices (40) as in the case of zigzag tubes, according to Eq. (19). For each nanotube line we have the following extrema points $\left(\gamma_{3}=\gamma_{1}\right)$ :

$$
\begin{gathered}
E_{l}^{1}=\sigma_{\mu}\left|\gamma_{1} \sin \frac{\pi l}{n}\right|, \quad k_{\|}= \pm \frac{2}{a} \operatorname{Arccos}\left(-\frac{\gamma_{1}}{2 \gamma_{2}} \cos \frac{\pi l}{n}\right), \\
E_{l}^{2}=\sigma_{\mu} \sqrt{\gamma_{1}^{2}+4 \gamma_{2}^{2}-4 \gamma_{1} \gamma_{2} \cos \frac{\pi l}{n}}, \quad k_{\|}=\frac{2 \pi}{a} \\
E_{l}^{3}=\sigma_{\mu} \sqrt{\gamma_{1}^{2}+4 \gamma_{2}^{2}+4 \gamma_{1} \gamma_{2} \cos \frac{\pi l}{n}}, \quad k_{\|}=0 .
\end{gathered}
$$

The position of these extrema in the folded zone of armchair tubes are shown in Fig. 9. The above three types of singularities arise in the energy regions $\left(0,\left|\gamma_{1}\right|\right), \quad\left(\mid 2 \gamma_{2}\right.$ $\left.-\gamma_{1} \mid, \sqrt{\gamma_{1}^{2}+4 \gamma_{2}^{2}}\right)$, and $\left(\sqrt{\gamma_{1}^{2}+4 \gamma_{2}^{2}},\left|2 \gamma_{2}+\gamma_{1}\right|\right)$ correspondingly [Fig. 9(b)]. The figure shows that the singularity points $E_{l}^{2}$ and $E_{l}^{3}$ are situated on the symmetry planes of the Brillouin zone. At the same time $E_{l}^{1}$ are located at nonsymmetric positions except for the line $l=n / 2\left(k_{\|}= \pm \pi / a\right)$ where it is situated in the $M$ point of the graphene's Brillouin zone, with a $C_{2}$ rotational symmetry. This means that the real part of $k_{\|}$, defining the oscillating behavior of the contribution from the corresponding nanotube line, will have constant values, given in Eq. (56), outside the corresponding band fragments containing one of the extrema $E_{l}^{2}, E_{l}^{3}, E_{n / 2}^{3}$ as a closest edge. On the contrary, in the case of $E_{l}^{1}$, $l \neq n / 2$ a gradual shift of $\operatorname{Re}\left\{k_{\|}\right\}$will emerge when the energy of transmission is swept outside the corresponding band fragment.

The curvature of the tube leads to the relation between transfer parameters ${ }^{52}\left|\gamma_{1}\right|<\left|\gamma_{2}\right|=\left|\gamma_{3}\right|$ for $(n, n)$ nanotubes [Fig. 1(a)]. The nonequivalence of transfer parameters is, however, not as crucial as in the case of zigzag tubes since the armchair tubes remain metallic. ${ }^{20}$ The only extrema which shift in the $k$ space with $\Delta \gamma=\left|\gamma_{1}-\gamma_{2}\right|$ are those located at the nonsymmetric positions in the Brillouin zone (Fig. 9). Contrary to zigzag tubes, $G_{11}^{0}, G_{i j}^{0}$ and the transmission itself will not vanish in the Fermi region of armchair tubes.

\section{Transmission at singularities}

The pairs of $\mathbf{k}_{s}$ points corresponding to a given singularity $(l \neq 0)$ of the second and third type, Eq. (56), can be related via the inversion operation in the Brillouin zone. Therefore, as in zigzag tubes, we can apply in this case Eqs. (38)

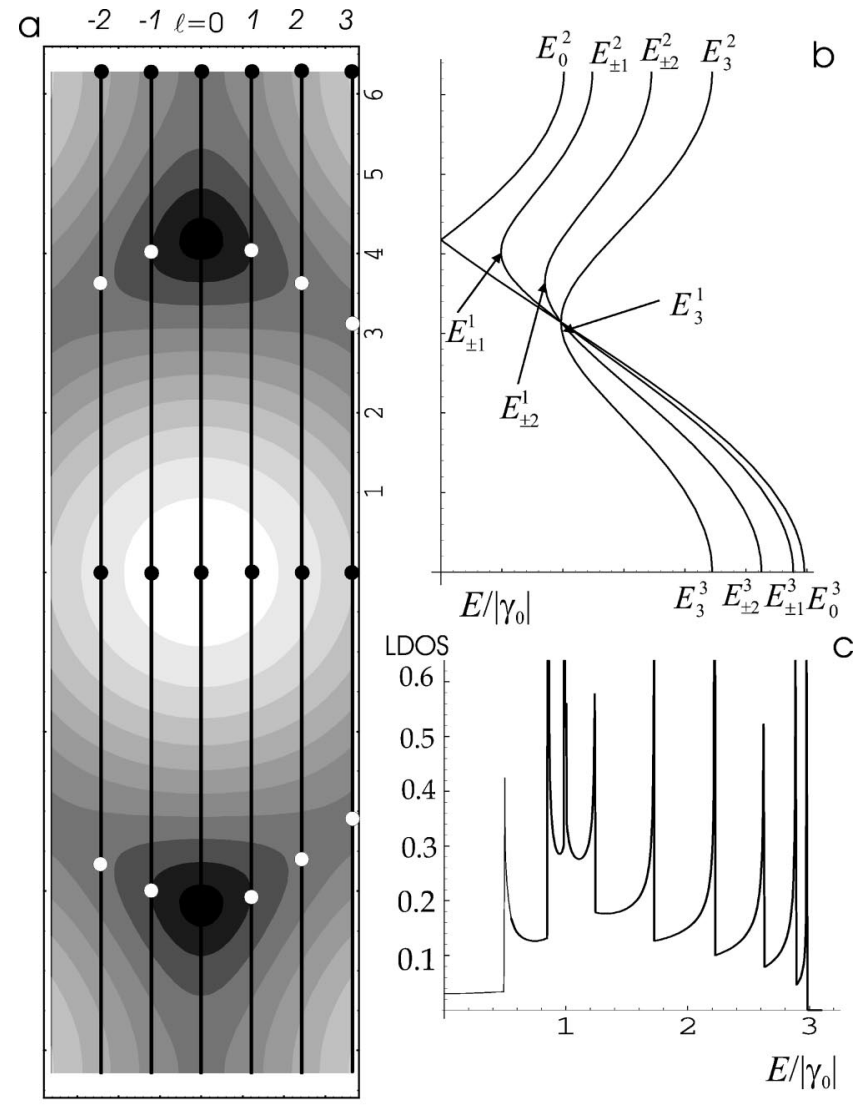

FIG. 9. Rectangular Brillouin zone (a), energy dispersion along the nanotube lines (b) and density of states per carbon atom (c) of the $(6,6)$ armchair nanotube. Solid straight lines in (a) stand for nanotube lines (numbered in italics), while the black and open circles show the position of singularities. The contour plot in (a) shows the lowest $\pi$ band of graphite in the rectangular Brillouin zone (dark regions indicate high energy). The energy labels in (b) show the position of the corresponding singularities. Only half of the energy spectrum is shown.

and (39) to find the conditions for nonzero transmission and missing peaks at singularities. In the case of contacted atoms of the same type this yields the conditions described by Eqs. (43) and (44), respectively, i.e., coinciding with those for zigzag tubes (now $n_{\perp}=n_{x}$ ). For contacted atoms of different type the condition of nonzero transmission at these singularities is only satisfied for the central nanotube line $l=0$, while the condition for missing peaks is never satisfied (again, as in the case of zigzag tubes, the transmission at the singularities of the $l=0$ line is always nonzero). Therefore the transmission peaks will be just split in this case at all singularities $E_{l}^{2,3}$ with $l \neq 0$.

On the other hand, as Fig. 9 shows, the $\mathbf{k}_{s}$ points corresponding to a given singularity of the first type $(l \neq 0, n / 2)$ cannot be related by the inversion operation in the Brillouin zone (there are more than two points for each singularity). In this case Eq. (38) is only a necessary condition for nonzero transmission, while Eq. (39) is only a sufficient condition for missing peaks at these singularities. With Eq. (56) for $k_{\|}$and 


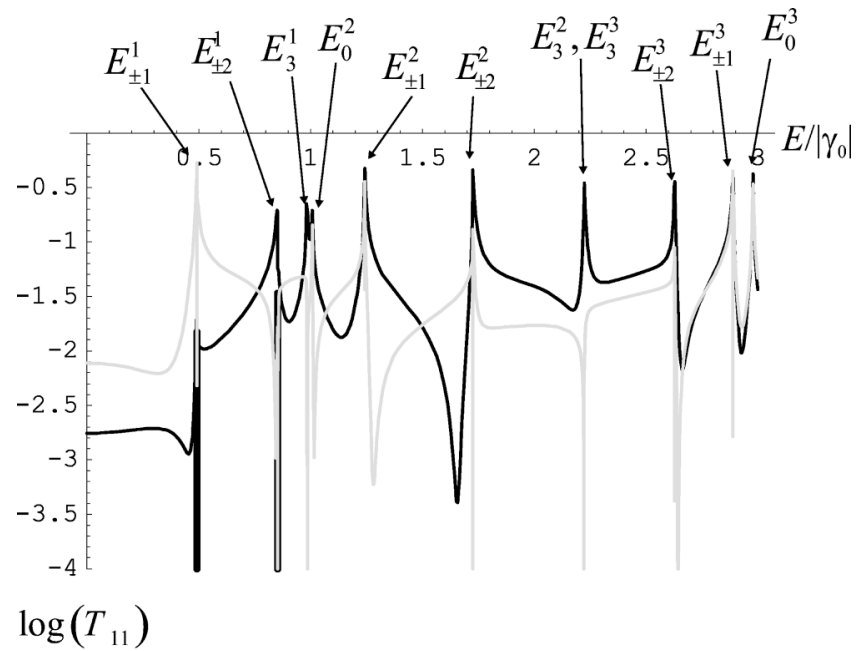

FIG. 10. Energy profile of the transmission through a $(6,6)$ nanotube (logarithmic scale) for two relative positions of the tips contacting atoms of the same type: $n_{\|}=4, n_{\perp}=0$ (solid line); $n_{\|}$ $=3, n_{\perp}=1$ (gray line). The coupling strength to the leads is $\Delta$ $=0.5\left|\gamma_{0}\right|$.

$$
k_{\perp}=\frac{2 \pi l}{\sqrt{3} a n},
$$

we conclude that the equations mentioned above are hardly satisfied for both equivalent and different type of contacted carbon atoms when $l \neq n / 2$. Moreover, we can check that while the condition in Eq. (39) is never satisfied for individual $\mathbf{k}_{s}$ points, it is not satisfied as a sum over the relevant $\mathbf{k}_{s}$ points either, i.e., the corresponding transmission peaks never can be missing ones at $E_{l}^{1}$ for $l \neq n / 2$. This means that the transmission function is always characterized by split peaks in these singularity points. In contrast, in the case $l$ $=n / 2$ Eqs. (38) are always satisfied for both equivalent and different type of contacted carbon atoms ( $f_{\mathbf{k}_{s}}$ is real). However, since the two, $\mathbf{k}_{s}$ points corresponding to the singularity $E_{n / 2}^{1}$ are not related by the inversion in the Brillouin zone [they are both lying on the same $l=n / 2$ nanotube line, Fig. $7(\mathrm{a})]$ this condition itself is not enough for nonzero transmission in this singularity. The additional condition to be satisfied is the equal value of RHS of Eq. (38) for both points, which is only the case when $n_{\perp}$ and $n_{\|}$are both even. At the same time, the condition in Eq. (39) for a missing peak at the $E_{n / 2}^{1}$ singularity is never satisfied for individual $\mathbf{k}_{s}$ points. However, it is satisfied as a sum over these points when $n_{\perp}$ and $n_{\|}$are both odd.

Figure 10 shows the energy dependence of the transmission for two relative positions of leads, similar to those used in Fig. 5 for the case of zigzag tubes. We can see that the transmission profiles differ drastically as a result of different fulfilment of the conditions in Eqs. (43) and (44) for these two geometries of attached leads. Indeed, going from $n_{\|}$ $=4, n_{\perp}=0$ to $n_{\|}=3, n_{\perp}=1$, the peaks at $E_{ \pm 1}^{2}, E_{ \pm 2}^{2}, E_{ \pm 2}^{3}$, and $E_{ \pm 1}^{3}$ turn from normal to splitted ones, while the normal peaks at $E_{3}^{1}, E_{3}^{2}$, and $E_{3}^{3}$ become missing. At the same time the peaks at the lowest two singularities $E_{ \pm 1}^{1}$ and $E_{ \pm 2}^{1}$ remain split since they correspond to singularities of the first type, while $E_{0}^{2}$ and $E_{0}^{3}$ remain normal peaks according to Eq. (43).

The behavior of the transmission at singularities as function of the distance between contacted carbon atoms is quite similar to zigzag tubes. The factor $\xi$ and the combination of the Green's functions $G_{11}^{0}-\xi G_{i j}^{0}$ entering the expression for the transmission in Table II are obtained as in the previous section. Particularly, in the limit $z \rightarrow E_{l^{\prime}}^{2,3}$ the term from the $l^{\prime}$ contribution, containing cancelling diverging parts, becomes

$$
G_{11}^{0}-\xi G_{i j}^{0} \approx \frac{1}{n}\left(2-\delta_{l^{\prime}, 0}\right) E_{l^{\prime}}^{2,3} \frac{\left|n_{\|}\right|}{4 \gamma_{2}\left(2 \gamma_{2} \mp \gamma_{1} \cos \frac{\pi l^{\prime}}{n}\right)}
$$

where the two signs in the denominator correspond to $E_{l^{\prime}}^{2}$ and $E_{l^{\prime}}^{3}$, respectively. At the singularity $E_{n / 2}^{1}$, the same term in the limit $z \rightarrow E_{n / 2}^{1}$ gives

$$
G_{11}^{0}-\xi G_{i j}^{0}=-\frac{E_{n / 2}^{1}}{n} \frac{\left|n_{\|}\right|}{4 \gamma_{2}^{2}} .
$$

We can see that the contributions (58) and (59) are linearly dependent on the distance between contacted carbon atoms as it was also the case for zigzag tubes, Eq. (48). However, they have a weaker dependence on the transmission energy as compared to Eq. (48). Other terms, corresponding to contributions from both $l^{\prime}$ and $l \neq l^{\prime}$ lines show decaying and oscillating behavior. Therefore at large distances between contacted atoms the transmission at singularities will decrease with distance as $\left|n_{\|}\right|^{-2}$ similarly to the case of zigzag tubes and monoatomic chains. Figure 11 shows the transmission at the singularities $E_{3}^{1}=\sigma_{\mu}\left|\gamma_{1}\right|$ and $E_{0}^{2}=\sigma_{\mu}\left|2 \gamma_{2}-\gamma_{1}\right|$ in the $(6,6)$ nanotube as function of the axial distance between contacted carbon atoms. We note a complex oscillation structure of the transmission function at low values of $\left|n_{\|}\right|$which is due to the interference of several nanotube lines which cross the point of this singularity (Fig. 9). At higher distances between the tips the transmission is of pure decaying type since only the terms (58) and (59) are contributing. The singularities with the lowest energy $|E|$ are of the first type [Fig. 9(b)], where the transmission peaks are split. However, for small enough values of the coupling strength to the leads this splitting is quite small, looking rather as a cut (Fig. 10). The intensity of these peaks decays with the distance between contacted atoms pretty much as for the ones with nonzero transmission at the singularity point (Fig. 11).

\section{Transmission in the Fermi region}

Consider now the transmission in the Fermi region, i.e., between $E=0$ (the Fermi level in undoped tubes) and the first singularity $E_{ \pm 1}^{1}$. The transmission at these energies is 
mainly determined by the contribution from the nanotube line $l=0$ crossing the Fermi points $K_{1}$ and $K_{2}$ of the graphene's Brillouin zone (Fig. 9a). The corresponding ex- pressions for the Green's functions in this energy region are derived in the Appendix D. At the Fermi level, substituting Eqs. (D2) into Eq. (33) and (34) we find

$$
T=\frac{\frac{2 \Delta^{2}}{3 n^{2} \gamma_{0}^{2}}\left(1-\frac{\Delta \gamma}{3\left|\gamma_{0}\right|}\right)^{2}\left(1 \pm \cos \left[2\left(\frac{\pi}{3}+\frac{\Delta \gamma}{\sqrt{3}\left|\gamma_{0}\right|}\right) n_{\|}\right]\right)}{\left[\left(1+\frac{1-\Delta \gamma / 3\left|\gamma_{0}\right|}{\sqrt{3} n\left|\gamma_{0}\right|}\right)^{2} \mp \frac{\Delta^{2}}{6 n^{2} \gamma_{0}^{2}}\left(1-\frac{\Delta \gamma}{3\left|\gamma_{0}\right|}\right)^{2}\left(1 \pm \cos \left[2\left(\frac{\pi}{3}+\frac{\Delta \gamma}{\sqrt{3}\left|\gamma_{0}\right|}\right) n_{\|}\right]\right)\right]^{2}}
$$

where the two signs correspond to the contacted atoms of the same and of different type, respectively. The contributions from other nanotube lines mutually cancel at $E=0$ when the leads are attached to atoms of the same type due to symmetry reasons discussed in the previous section, while they are nonzero for atoms of different types.

At small values of $\left(\Delta / \gamma_{0}\right)^{2}$ the distance dependence of the transmission is mainly determined by the last factor in the numerator of Eq. (60). When the curvature effects are neglected $(\Delta \gamma=0)$, this factor can only take two values in the whole range of $n_{\|}: 2,1 / 2$ for atoms of the same type and
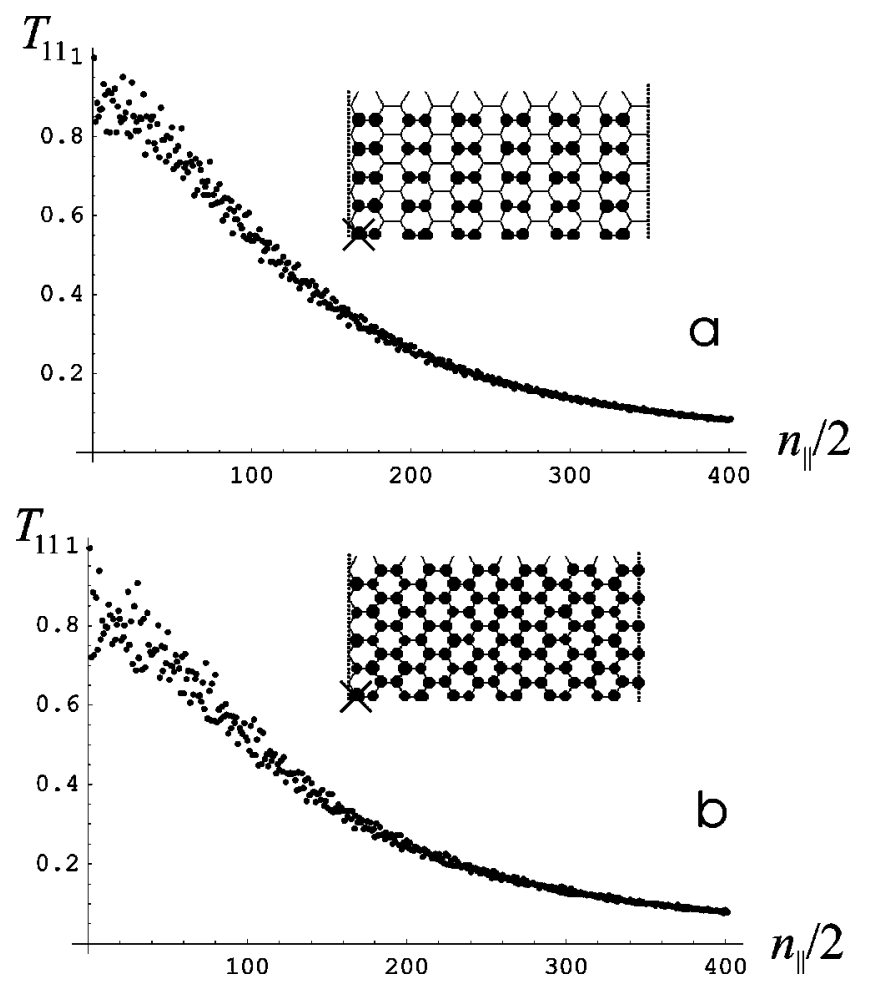

FIG. 11. Nonzero transmission at the singularites $E_{3}^{1}$ (a) and $E_{0}^{2}$ (b) in the $(6,6)$ nanotube (Fig. 9) as function of axial distance between contacted carbon atoms of the same type. The coupling strength to the leads is $\Delta=0.1\left|\gamma_{0}\right|$. The inset shows the map of possible positions of monoatomic contacts on the surface of the nanotube's strip corresponding to nonzero transmission; the cross shows the position of the first tip and one of the black circles the position of the second tip.
$0,3 / 2$ for atoms of different type, the second value being met twice as often as the first one. However, as Fig. 12(a) shows, inclusion of curvature effects radically changes this picture even if $\Delta \gamma / \gamma_{0}$ is quite small, of the order of 0.01. Indeed, the distance dependence of the transmission is described in this case by an alternating function, which oscillates between three harmonic branches, whose period is inverse proportional to $\Delta \gamma / \gamma_{0}$. The origin of the $\Delta \gamma$ terms in the arguments of harmonic functions in the above equation and also in Eqs. (D2) and (D3) can be traced back via Eqs. (26) and (20) to $k_{\|}$values at the Fermi level. These are $\mp 2 \pi / 3 a$, i.e., correspond to the $K_{1,2}$ points in the graphene's Brillouin zone [Fig. 1(b)] in the absence of curvature effects, but acquire a shift along the nanotube line $l=0$ proportional to $\Delta \gamma / \gamma_{0}$ when curvature effects are taken into account. ${ }^{60}$ Note that this was not the case for zigzag nanotubes where the curvature-induced shift of the Fermi points along $k_{\|}$is exactly zero and therefore no long period oscillations of the transmission as function of axial distance between the leads have been found (see the next section for further discussion).

In the case of atoms of different type the contributions to $G^{0}$ from other nanotube lines, first of all from $l= \pm 1$, show up already at $E=0$. Moreover, Eq. (D7) shows that the prefactor in this contribution is $2 n / \pi$ times larger than in the $l$ $=0$ contribution, Eq. (D2). However, the contributions from the lines $l \neq 0$ will decay fast with the axial distance between the leads, so that the behavior of the transmission function will be qualitatively the same as in the case of atoms of the same type.

When the energy deviates from $E=0$, the transmission is modified mainly as a change of the period of oscillations of the three harmonic components [Figs. 12(b)-12(e)]. This is due to the energy dependence of the angle $\varphi$, Eq. (D3), in the argument of harmonic functions entering the expression for $G^{0}$ in Eq. (D2). According to this energy dependence, the increase of $|E|$ will lead first to the increase of the period of oscillation of the harmonic functions since the deviation of $\varphi$ from $\pi / 3$ decreases. At some energy this deviation will reduce to zero, which means that the transmission function has only two values for the absence of curvature effects discussed above. The corresponding energy point is easily found from the first equation in (D3):

$$
\left|E_{0}\right| \approx \sqrt{6 \Delta \gamma\left|\gamma_{0}\right|}
$$



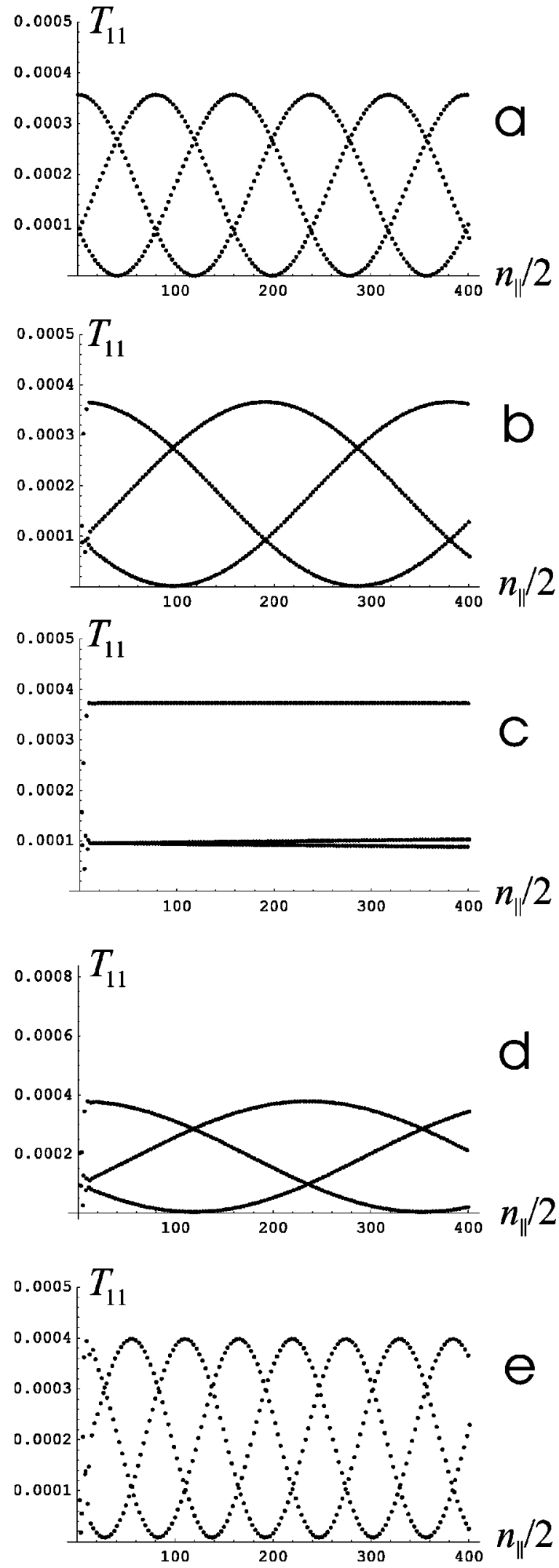

FIG. 12. Distance dependence of the transmission in the Fermi region of a $(6,6)$ nanotube for axial shift between contacted atoms of the same type $\left(n_{\perp}=0\right)$ at energies of transmission: $\left|E / \gamma_{0}\right|=0$ (a), 0.2 (b), 0.26 (c), 0.3 (d) and 0.4 (e). The coupling strength to the leads is $\Delta=0.1\left|\gamma_{0}\right|$.
Further increase of energy from the $\left|E_{0}\right|$ point will gradually decrease the period of oscillation of the three harmonic components since a growing deviation (now a negative one) of $\varphi$ from $\pi / 3$ will emerge. This is exactly what is observed in Figs. 12(b)-12(e). The plot (c) in this figure shows the distance dependence of the transmission at the energy $\left|E_{0}\right|$ given by Eq. (61). Note that the last relation is obtained from an approximate expression for $\varphi$, which only includes lowest order energy corrections, Eq. (D3).

The contributions from "nonmetallic" nanotube lines $l$ $\neq 0$ to $G^{0}$ will increase with the deviation from the $E=0$ point. The most important of them, corresponding to $l$ $= \pm 1$, Eqs. (D7), will have the $\epsilon\left(1-\epsilon^{2}\right)^{-1 / 2}$ dependence on the dimensionless energy $\epsilon$, defined in Eqs. (D6), for contacted atoms of the same type and $\left(1-\epsilon^{2}\right)^{-1 / 2}$ dependence for atoms of different types. For the large part of the domain $0 \leqslant \epsilon<1$ this contributions show up in the distance dependence of the transmission function only for values of $n_{\|}$ $<10$, Figs. 12(b) $-12(\mathrm{e})$. By contrast, the contribution from the "metallic" nanotube line is only weakly dependent on the energy which is confirmed by an almost constant amplitude of the transmission function in the wide range of energy deviations from $E=0$.

The above picture begins to change when the energy gets into vicinity of the first singularity $E_{ \pm 1}^{1}$ (Fig. 13). First, the range of $n_{\|}$values where the contribution of $l= \pm 1$ lines is significant increases. This is the result of a slower decay of this contribution with the axial distance between contacted atoms, due to a smaller factor $\left(1-\epsilon^{2}\right)$ in the exponent of $G^{0}$ in Eq. (D7). Second, the same factor in the denominator of the $l= \pm 1$ contribution in Eq. (D7) causes the strong increase of the amplitude of the transmission function. At the same time in the region of large $n_{\|}$, where the contributions of $l \neq 0$ lines become unimportant, the transmission still keeps the same amplitude as in the previous cases, shown in Fig. 12. However, for transmission energies close to $E_{ \pm 1}^{1}$, when $G_{11}^{0}$ in the denominator of the expression for $G_{i j}$ in Eq. (34) becomes large enough, ${ }^{61}$ the amplitude of the transmission determined by the $l=0$ contribution begins to decrease in the region of large $n_{\|}$, which is observed in Fig. 13(b). Finally the transmission disappears in the limit $E=E_{1}^{1}$ as it should according to the rules established above for transmission at singularities of this type.

\section{Chiral nanotubes}

The simplest chiral nanotubes (with most pronounced chirality) are of the type $(2 n, n)$ since they have reduced indices $n^{\prime}=2, m^{\prime}=1$. These give the smallest possible sum, $n^{\prime}+m^{\prime}=3$, and therefore the lowest possible degree (six) of the polynomial $p(w)$ in Eq. (28). Even for this type of chiral tubes complete analytical solutions for the Green's functions can be found only for $n=1,2$. Except for these simple cases and several others, specified in Sec. II B, the zeroes of $p(w)$ should be found numerically and substituted into Eqs. (30) and (31).

To find the singularities for chiral nanotubes one has generally to solve the equation $d E_{\mu \mathbf{k}} / d k_{\|}=0$ for each nanotube line. If, however, the nanotube lines cross some symmetry 


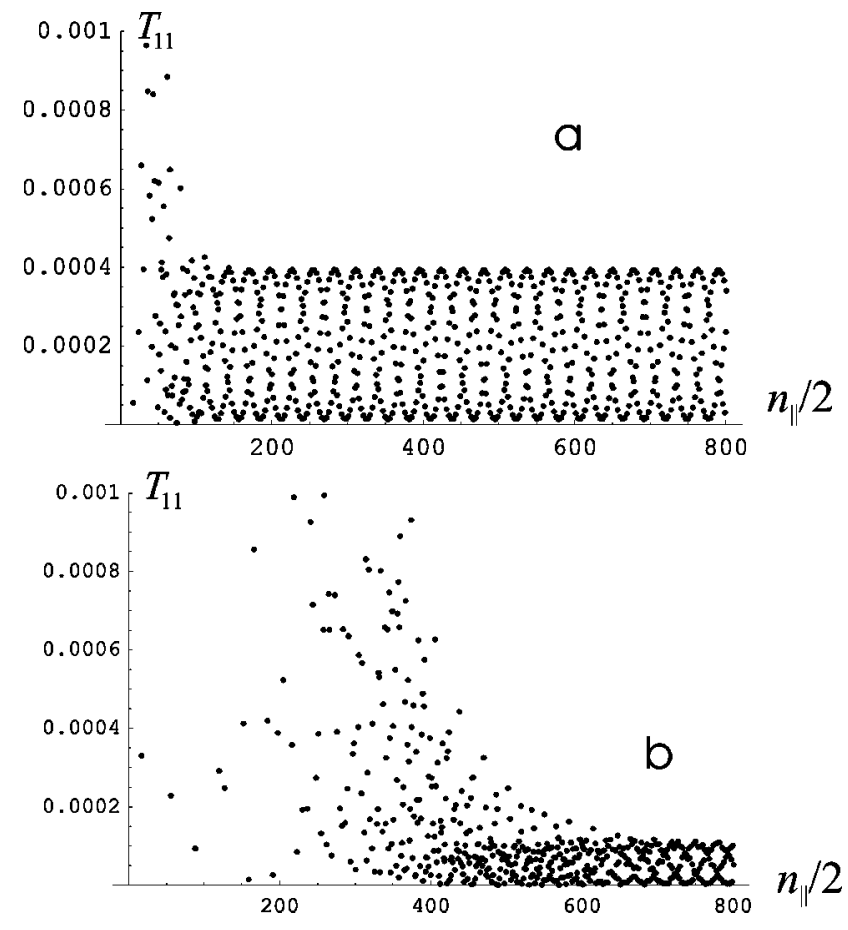

FIG. 13. Distance dependence of the transmission close to the first singularity $E_{ \pm 1}^{1}$ of a $(6,6)$ nanotube for axial shift between contacted atoms of the same type $\left(n_{\perp}=0\right)$ at energies of transmission: $\left|E / \gamma_{0}\right|=0.49$ (a) and 0.4923 (b). The coupling strength to the leads is $\Delta=0.1\left|\gamma_{0}\right|$. The first singularity is found at $\left|E / \gamma_{0}\right|$ $=0.49235$.

points of the folded Brillouin zone, the last obviously become singularities. In arbitrary $(n, m)$ tubes this is always the case for the $\Gamma$ point $E=\sigma_{\mu}\left|\gamma_{1}+\gamma_{2}+\gamma_{3}\right|$, for the $M_{1}$ point ( $m$ even) $E=\sigma_{\mu}\left|-\gamma_{1}-\gamma_{2}+\gamma_{3}\right|, M_{2}$ point $(n$ even) $E$ $=\sigma_{\mu}\left|-\gamma_{1}+\gamma_{2}-\gamma_{3}\right|$, and $M_{3}$ point $(n+m$ even $) \quad E$ $=\sigma_{\mu}\left|\gamma_{1}-\gamma_{2}-\gamma_{3}\right|[$ [Fig. 1(b)]. Note that only an odd number of $M$ points (one or three) can be intersected in any kind of tubes. ${ }^{45}$ We can see that any of the three $M$ points can be the only intersected one, which however corresponds to the same point in the rectangular Brillouin zone of the chiral tube, always located at the end of the line $l=0$ (Fig. 14). This single point corresponds to $M_{1}$ for $n^{\prime}$ even, $m^{\prime}$ odd, to $M_{2}$ for $n^{\prime}$ odd, $m^{\prime}$ even, and to $M_{3}$ for both $n^{\prime}, m^{\prime}$ odd. The other two $M$ points are only present in the folded Brillouin zone when $n$ and $m$ are both even, and are located on the line $l=\operatorname{GCD}(n, m) / 2$ which lies on the edge of the rectangular Brillouin zone (if present). Note that all relevant $M$ points are found at the edge of the rectangular Brillouin zone since these points cannot be related by inversion with respect to the central $(\Gamma)$ point [see Fig. 1(b)].

The number of extrema along each nanotube line is $2\left(n^{\prime}+m^{\prime}\right)$. The $k_{\|}$projection of the wave vector, defining the pole via Eqs. (20) and (26), either corresponds to the intersection point of $E$ with a given band fragment confined between two extrema (edges) or has its real part around the value at the closest edge of this band region when the transmission energy is swept outside. The real part of $k_{\|}$will coincide with the last value only if the corresponding singularity is a symmetry point of the folded Brillouin zone, i.e.,

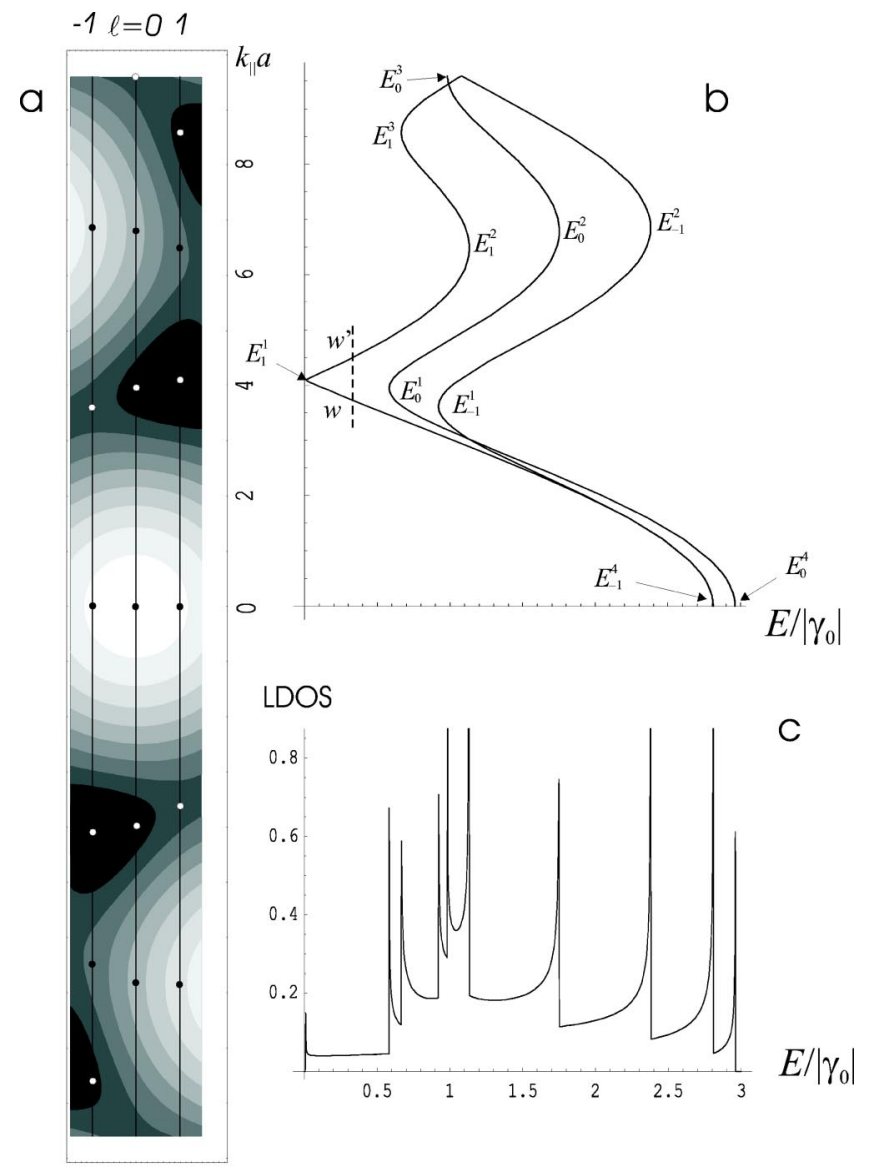

FIG. 14. Rectangular Brillouin zone (a), energy dispersion along the nanotube lines (b) and density of states per carbon atom (c) of the $(6,3)$ chiral nanotube. Solid straight lines in (a) stand for nanotube lines (numbered in italics), while open and filled circles show the position of singularities. The contour plot in (a) shows the lowest $\pi$ band of graphite in the rectangular Brillouin zone (dark regions indicate higher energies). The energy labels in (b) show the position of the corresponding singularities. Only half of the energy spectrum is shown in (a-c). The intersections of the dashed line with the bands show the position of the poles $w$ and $w^{\prime}$.

one of the $\Gamma, M_{1}-M_{3}$ points. At the same time most of the extrema in the Brillouin zone of chiral tubes are situated at nonsymmetric positions [Fig. 14(a)]. Due to the stretching of the Brillouin zone in the $k_{\|}$direction many extrema arise along each nanotube line in this type of tubes. Even in $(2 n, n)$ tubes we have already four types of extrema for each nanotube line [Fig. 14(b)], i.e., more than in zigzag and armchair tubes. Another difference from achiral tubes is the lack of reflection symmetry $k_{\|} \rightarrow-k_{\|}$within one nanotube line for $l \neq 0$. However, due to the inversion symmetry with respect to the center of the Brillouin zone, the mirror symmetry with respect to the $k_{\|}=0$ plane is restored for the whole band structure but corresponds now to the reflection of the $l$ bands into the $-l$ ones. This symmetry together with the translation symmetry of the opposite short edges of the Brillouin zone merges the $l$ and $-l$ bands at these edges into one point [Fig. 14(b)].

The curvature of the tube makes all three transfer parameters unequal to each other, which opens a gap at the Fermi 
level in all primary metallic tubes, with the difference of the nanotube indices $n-m$ divisible ${ }^{34}$ by 3, Fig. 14(c). Although in these tubes the nanotube lines cross the Fermi points, these do not correspond to singularities anymore, unlike in the case of zigzag tubes, since $K_{1}$ and $K_{2}$ lose rotational symmetry in the folded Brillouin zone of chiral tubes. Hence the singularities shift away from the Fermi points along the corresponding nanotube lines.

\section{Transmission at singularities}

The $\mathbf{k}_{s}$ points of a general position corresponding to a given singularity are met in pairs related by inversion, so we can apply Eqs. (38) and (39) to check the conditions for nonzero transmission and missing peaks at these singularities. It is clear, however, that both of these conditions are hardly satisfied given the nonsymmetric position of $\mathbf{k}_{s}$ points in the Brillouin zone for singularities of this type [Fig. 14(a)]. Therefore the transmission peaks will be split at all these singularities for any relative positions of the contacted atoms. On the other hand, the transmission at singularities corresponding to the symmetry points mentioned above is always nonzero for any relative positions of the leads, which can be checked straightforwardly by introducing the coordinates of the $\Gamma$ and $M_{i}$ points [Fig. 1(b)] into Eqs. (38) and (39). The energies of singularities at $M_{i}$ points (if intersected) are all different but in the armchair limit the two of them, which lie on the line $l=\operatorname{GCD}(n, m) / 2$, become equivalent. As a consequence of interference of these two points the transmission peak can be missing at the corresponding singularity at some values of $n_{\|}$and $n_{\perp}$ (see the previous section).

The distance dependence of transmission at singularities is quite similar to the case of achiral tubes, Figs. 4 and 11. In particular, it is inverse proportional to the square of the distance between the leads shifted in the axial direction, when these are far enough from each other.

\section{Transmission in the gap region}

In the gap region, in the middle of the band structure ( $E$ $\approx 0$ ), the transmission function exhibits the same qualitative behavior as in zigzag tubes. At $E=0$ the transmission between atoms of the same type is exactly zero for tubes of any chirality. However, for contacted atoms of different type we face the same phenomenon of directional anisotropy of electron transmission (a kind of rectifying effect) as found already in zigzag tubes, Eq. (51). Indeed, fixing one of the leads on an arbitrary carbon atom we see a nonzero transmission in one direction along the tube's axis and an almost suppressed transmission in the opposite direction (Fig. 15). The difference from zigzag tubes is that now the "nonzero" side shows a highly inhomogeneous map of transmission. The distance dependence of the transmission in the gap region is shown in Fig. 16 for the $(6,3)$ tube as function of the axial shift measured in units of the translation vector $\mathbf{T}$ (Fig. 15). As in zigzag tubes, we see that the transmission has the lowest amplitude and shows the fastest decay in the middle of the gap $(E=0)$, while it increases and becomes less decaying when approaching the first singularity at the Fermi

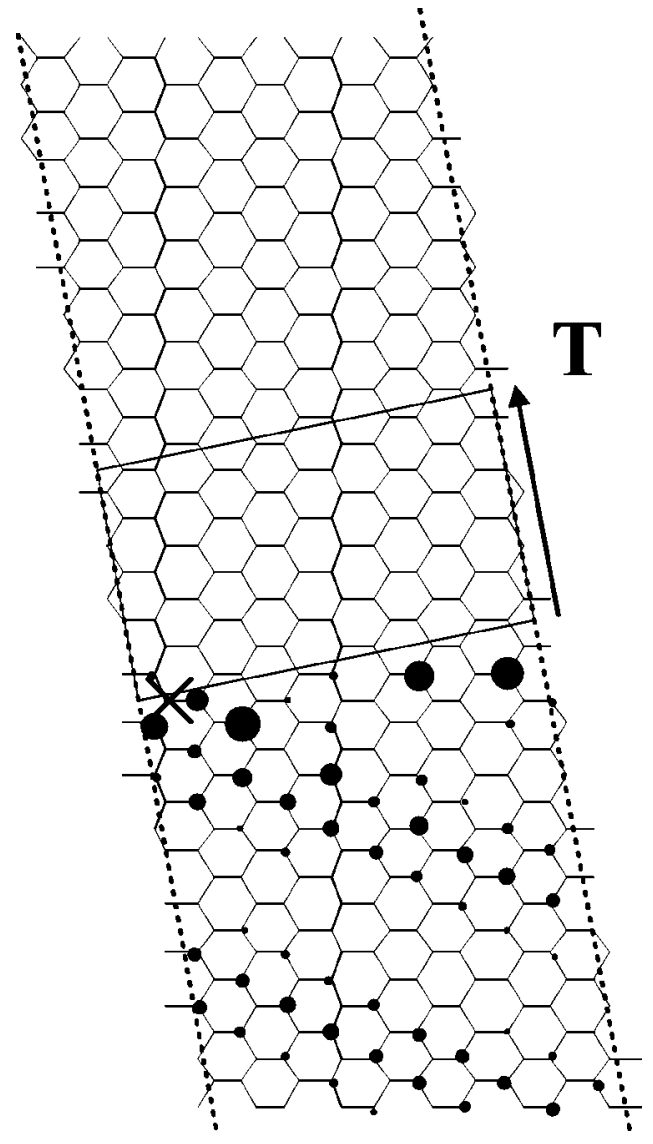

FIG. 15. A graphene's strip of an unrolled $(6,3)$ nanotube (dashed lines) and the corresponding map of transmission at $E=0$ (filled circles). The segment perpendicular to the strip delineates the smallest unit which can be translated along the tube's axis, $\mathbf{T}$ being the corresponding translation vector. The cross shows the position of the first lead and the size of the filled circles scales the value of the transmission through the corresponding carbon atoms.

level. The main difference from zigzag tubes is that now the transmission in the gap shows an oscillating-decaying behavior. Reaching the Fermi energy, the transmission goes to zero since the transmission peak is split at the corresponding singularity. In the Fermi region, which includes the gap and the band regions till the second singularity ( $E_{0}^{1}$ in Fig. 14), the transmission is mostly determined by the contributions from the "metallic" lines crossing the $K_{i}$ points. The condition for the "metallicity" of a nanotube line in the rectangular Brillouin zone of arbitrary tubes reads as

$$
l^{\prime}= \begin{cases}0 & \text { if } \frac{n^{\prime}-m^{\prime}}{3}=\text { integer } \\ \pm p & \text { if }(n, m)=\left(3 p n^{\prime}, 3 p m^{\prime}\right),\end{cases}
$$

where $p$ is an integer and it is understood that $n^{\prime}-m^{\prime}$ is not divisible by three in the second relation.

\section{Transmission in the band region}

Going beyond the first singularity ( $E_{1}^{1}$ in Fig. 14) into the band region, the amplitude of the transmission function stabilizes, while the distance dependence in the axial direction 


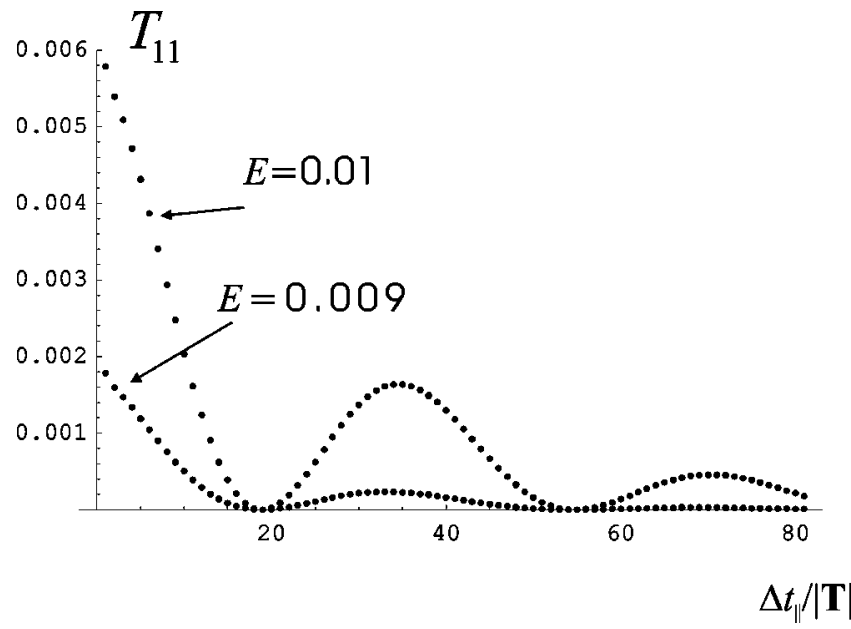

FIG. 16. Transmission in the gap region close to the Fermi level in a $(6,3)$ nanotube between atoms of the same type shifted relative to each other in the axial direction by an integer number of translational units. The number at each line denotes the energy of transmission in units of $\left|\gamma_{0}\right|$ and the coupling strength to the leads is $\Delta=0.1\left|\gamma_{0}\right|$.

(in units of the translation vector $\mathbf{T}$ ) shows (Fig. 17) a very similar behavior to the one found in armchair tubes (Fig. 12). Indeed, we see again oscillations of the transmission, which period increases with the energy deviation from the Fermi level, passes by the maximum (infinity) and then starts to decrease. At the end of the Fermi region, in the vicinity of the second singularity, the transmission function changes qualitatively in a similar way as discussed for armchair tubes (Fig. 13). However, in contrast to those tubes, the oscillations of transmission in Fig. 17 are described by one single harmonic function, which is certainly due to the fact that we followed larger steps, of translational unit $|\mathbf{T}|$. If one is trying smaller steps and various directions for the shift vector $\Delta \mathbf{t}$, very different patterns of the transmission as a function of the distance between contacted atoms emerge. There are, however, special directions for which the transmission can be described as a superposition of a few long-period harmonic components. For instance, in the case of $(2 n, n)$ nanotubes one of these directions corresponds to $\Delta \mathbf{t}$ aligned to a zigzag line parallel to the $y$ axis [Fig. 1(a)]. Following steps of minimal separation between carbon atoms of the same type we obtain for this direction a transmission function corresponding to three equidistantly shifted harmonic components which looks very much as the transmission of armchair tubes in the axial direction (Fig. 12).

\section{The analytical treatment}

To understand these results we investigate below the transmission in the band region for small $|E|$ analytically. Actually to describe the distance dependence of the transmission we only need to know the contributing poles entering the numerator in Eqs. (30) and (31). These are related to $k_{\|}$ via Eqs. (26) and (20) which, in turn, is found as a solution of $E=\sigma_{\mu} E_{k_{\|} l}$ when $E$ falls into the band region. The latter equation is a particular case of $p(w)=0$, so, as mentioned
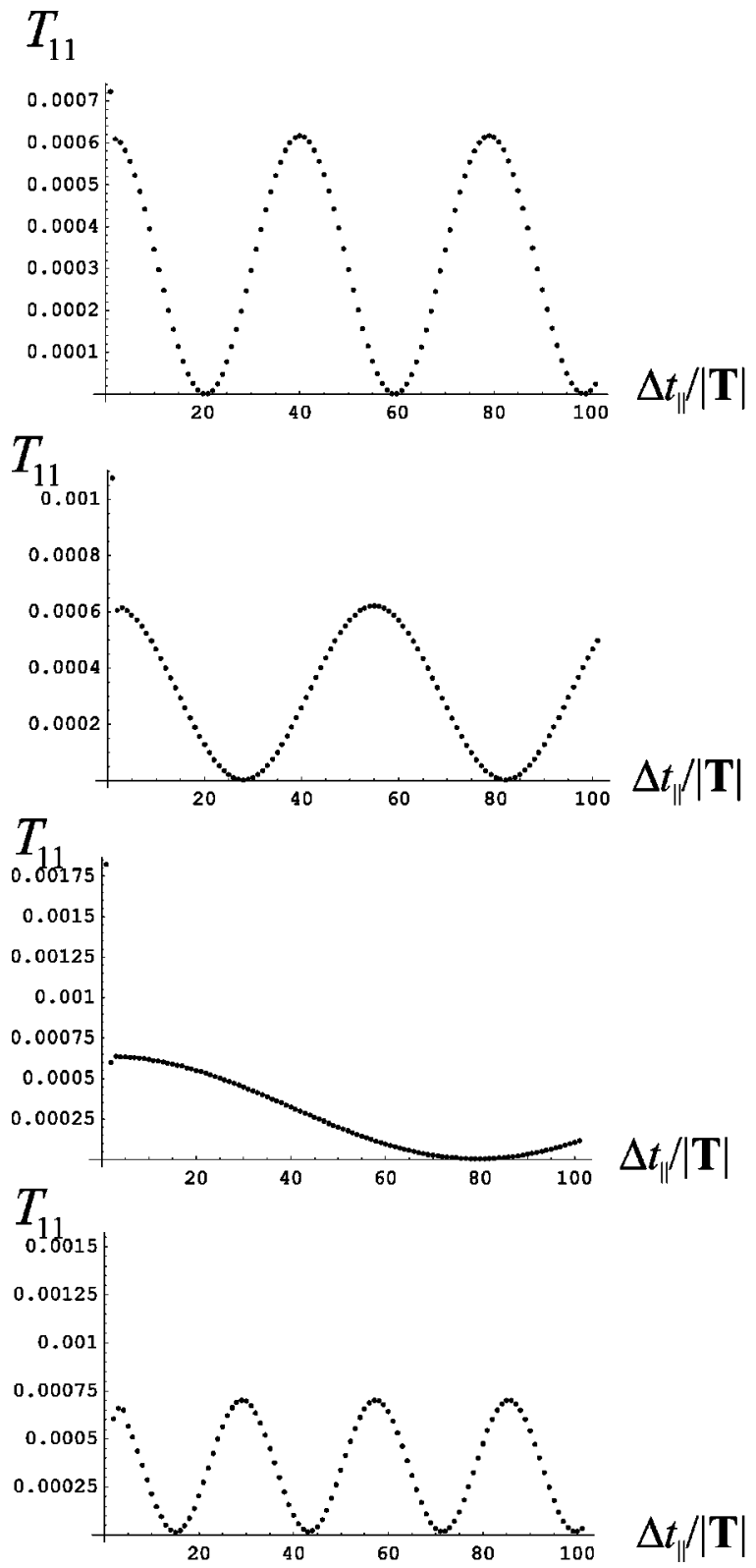

FIG. 17. Distance dependence of the transmission in the Fermi region of a $(6,3)$ nanotube for axial shift between contacted atoms of the same type (in steps of the translational unit) at energies of transmission: $\left|E / \gamma_{0}\right|=0.01$ (a), 0.1 (b), 0.2 (c), and 0.5 (d). The coupling strength to the leads is $\Delta=0.1\left|\gamma_{0}\right|$.

above, both of them cannot be solved analytically in the case of arbitrary chiral tubes. However, we can find approximate solutions for values of the transmission energy not too close to the second singularity ( $E_{0}^{1}$ in Fig. 14). Then, as it was also the case for achiral tubes, the main contributions to $G^{0}$ and the transmission as a whole come from "metallic" nanotube lines, e.g., $l=1,-1$ in Fig. 14(a). In the case of chiral and armchair nanotubes the two contributing poles are chosen among the four: $w, w^{\prime}$ from the vicinity of one of the Fermi points $K_{i}$ [shown as intersections with the dashed line in Fig. 14(b)] and $w^{-1}, w^{\prime-1}$ corresponding to another Fermi point, obtained by the inversion of the former. In the hexagonal Brillouin zone these poles correspond to 

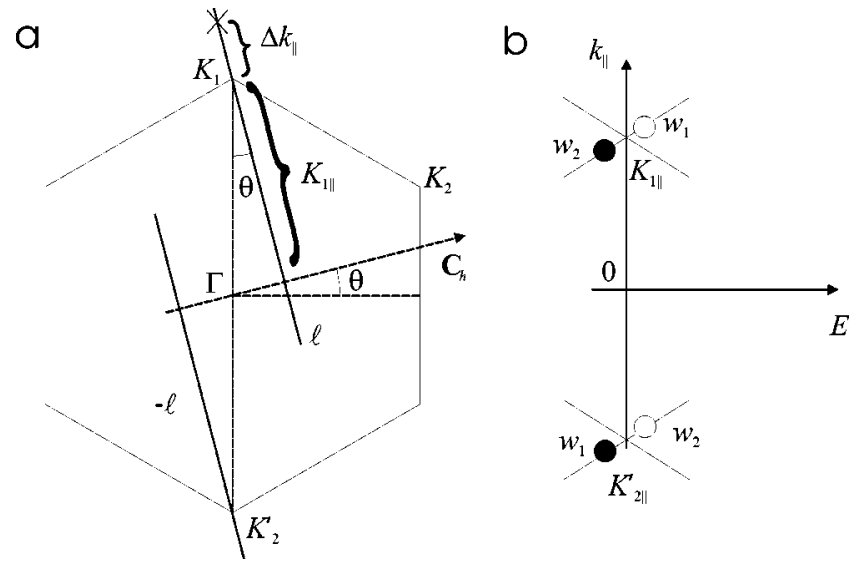

FIG. 18. The poles of $G^{0}$ giving the main contribution to the transmission through primary metallic nanotubes in the Fermi region. (a) the position of one of the poles (cross) on the "metallic" nanotube line $l$ in the hexagonal Brillouin zone; (b) the positions of the contributing poles in the band structure for negative (filled circles) and positive (empty circles) energies of transmission.

$$
\begin{aligned}
& k_{\|}=K_{1 \|}-\left|\Delta k_{\|}\right|, \\
& k_{\|}^{\prime}=K_{1 \|}+\left|\Delta k_{\|}\right|,
\end{aligned}
$$

where $K_{1 \|}$ is the projection of the Fermi point $K_{1}$ on the tube's axis and $\Delta k_{\|}$is the deviation from $K_{1}$ along the nanotube line crossing this point, Fig. 18(a).

After some transformations we obtain for the contributing poles (i.e., lying inside the unit circle) the following expressions:

$$
\begin{aligned}
w_{1,2}= & \exp \left( \pm i \operatorname{sgn}(E) \frac{\cos \theta}{n^{\prime}+m^{\prime}}\left\{\frac{4 \pi}{3} \cos \theta \pm \frac{2}{\sqrt{3}}\left|\frac{E}{\gamma_{0}}\right|\right.\right. \\
& +\operatorname{sgn}(\gamma) \frac{2}{\sqrt{3}\left|\gamma_{0}\right|}\left[\Delta \gamma_{1} \cos \theta+\Delta \gamma_{2} \cos \left(\theta+\frac{2 \pi}{3}\right)\right. \\
& \left.\left.\left.+\Delta \gamma_{3} \cos \left(\theta-\frac{2 \pi}{3}\right)\right]+\frac{1}{3 \sqrt{3}}\left(\frac{E}{\gamma_{0}}\right)^{2} \cos (3 \theta)\right\}\right),
\end{aligned}
$$

where $\Delta \gamma_{i}=\gamma_{i}-\gamma_{0}$ and the two signs in the exponent correspond to two subscripts of $w$. The position of these poles in the band structure is shown in Fig. 18(b). Substituting the data for armchair tubes, $n^{\prime}=m^{\prime}=1, \theta=0, \Delta \gamma_{2}=\Delta \gamma_{3}$, and using the relation $\left|\Delta \gamma_{1}\right|>\left|\Delta \gamma_{2}\right|$ as previously, ${ }^{52}$ we obtain for $w_{1,2}$ the expressions coinciding respectively with $w_{1(2)-, 0}$ in Eq. (D1). Substituting then $n^{\prime}=1, m^{\prime}=0, \theta=\pi / 6$ and the symmetry relation $\Delta \gamma_{1}=\Delta \gamma_{2}$ which holds for $(n, 0)$ zigzag tubes, we see that the last two terms in the exponent of Eq. (64) disappear, so we obtain one single pole $w=$ $-\exp \left(i E /\left|\gamma_{0}\right|\right)$, coinciding with $w_{+, n / 3}$ in Eq. (C3) in the lowest, zero order after $\Delta \gamma$. The last equation is more exact than Eq. (64) in the region close to the first singularity $|E|$ $\approx \Delta \gamma$.

Now we are in the position to evaluate the distance dependence of the transmission in the band region of chiral tubes. The contribution of the "metallic" lines $l$ ', $-l$ ' $(l$ ' $\geqslant 0$ ) to $G^{0}$ in Eqs. (30) and (31) can be written in the following general form:

$$
\begin{aligned}
G^{0}(\Delta \mathbf{t}, i j ; E)= & \widetilde{C}_{1} \mathrm{e}^{2 i \pi\left(l^{\prime} / q\right) \operatorname{sgn}(n \|) n_{\perp}} w_{l^{\prime}}^{\left|n_{\|}\right|} \\
& +\widetilde{C}_{2} \mathrm{e}^{-2 i \pi\left(l^{\prime} / q\right) \operatorname{sgn}\left(n_{\|}\right) n_{\perp} w_{-l^{\prime}}^{\left|n_{\|}\right|},}
\end{aligned}
$$

where $q$ is defined in Eq. (25) and $\widetilde{C}_{1}$ and $\widetilde{C}_{2}$ are complex coefficients independent from $n_{\|}, n_{\perp}$. Next we assign the poles entering Eq. (65) to $w_{1,2}$ in Eq. (64). For values of chiral angle within the range $0 \leqslant \theta \leqslant \pi / 6$, the bands in the vicinity of the $K_{1}$ point will be intersected by the nanotube lines with positive line index $l^{\prime}$ [Figs. 14(b) and 18(a)], while those belonging to $K_{2}\left(K_{2}^{\prime}\right)$ by its partner, $-l^{\prime}$. Hence from Fig. 18(b) we find the following correspondence:

$$
w_{l^{\prime}}= \begin{cases}w_{1}, & E>0, \\ w_{2}, & E<0,\end{cases}
$$

and the opposite for $w_{-l^{\prime}}$. Then we obtain for the transmission

$$
\begin{aligned}
T \sim & \left|G^{0}(\Delta \mathbf{t}, i j ; E)\right|^{2} \\
= & \mid\left(\widetilde{C}_{1}+\widetilde{C}_{2}\right) \cos \left(2 \pi \frac{l^{\prime}}{q} \operatorname{sgn}\left(n_{\|}\right) n_{\perp}+\tilde{\varphi}\left|n_{\|}\right|\right) \\
& +\left.i\left(\widetilde{C}_{1}-\widetilde{C}_{2}\right) \sin \left(2 \pi \frac{l^{\prime}}{q} \operatorname{sgn}\left(n_{\|}\right) n_{\perp}+\tilde{\varphi}\left|n_{\|}\right|\right)\right|^{2},
\end{aligned}
$$

where

$$
\begin{aligned}
\tilde{\varphi}= & \frac{\cos \theta}{n^{\prime}+m^{\prime}}\left\{\frac{4 \pi}{3} \cos \theta+\operatorname{sgn}(\gamma) \frac{2}{\sqrt{3}\left|\gamma_{0}\right|}\left[\Delta \gamma_{1} \cos \theta\right.\right. \\
& \left.+\Delta \gamma_{2} \cos \left(\theta+\frac{2 \pi}{3}\right)+\Delta \gamma_{3} \cos \left(\theta-\frac{2 \pi}{3}\right)\right] \\
& \left.+\frac{1}{3 \sqrt{3}}\left(\frac{E}{\gamma_{0}}\right)^{2} \cos (3 \theta)\right\} .
\end{aligned}
$$

It follows from Eq. (67) that all primary metallic chiral tubes can be divided in two groups. The first one, characterized by the "metallic" index $l^{\prime}=0$, shows roughly no dependence of the transmission on $n_{\perp}$. By contrast, the second group, with $l^{\prime} \neq 0$, demonstrates such a dependence. Returning back to Eq. (62) we observe that the two groups of chiral tubes are characterized in addition by different values of $R: 3$ for the first and 1 for the second group. It has been shown ${ }^{42}$ that nanotubes with different factors $R$ belong to different classes of line groups.

\section{The universal behavior of the transmission}

With Eq. (67) in hand we can easily explain the obtained numerical results for the distance dependence of the transmission. Substituting the $n_{\|}, n_{\perp}$ coordinates of the translation vector T from Table I into Eq. (67), we find that the main contribution to the argument of harmonic functions, arising 
from the term $\sim 4 \pi / 3$ in Eq. (68) is equal to $(2 / R)\left(n^{\prime}\right.$ $\left.+m^{\prime}\right) \pi$. Clearly this term is a multiple to $2 \pi$ for chiral tubes from the second group $(R=1)$ including zigzag tubes, therefore it does not contribute to the oscillations of the transmission when the distance between leads is changed in steps of the translational unit along the tube. The only contributions to the oscillations of the transmission function arise in this case from the small terms in Eq. (68), $\sim \Delta \gamma_{i}$ and $\sim\left(E / \gamma_{0}\right)^{2}$. Therefore in primary metallic tubes of the second kind the transmission is described by one long period harmonic function. This is precisely what we have obtained numerically for the (6,3) tube (Fig. 17). On the other hand in primary metallic tubes of the first kind $(R=3)$, including armchair tubes, the resulting main term $(2 / R)\left(n^{\prime}+m^{\prime}\right) \pi$ in the arguments of the harmonic functions is a multiple to $2 \pi / 3$. This means that the transmission function is described in this case by three long period harmonic components shifted by $2 \pi / 3,4 \pi / 3$, and $2 \pi$ correspondingly, the slow oscillation in each of them being determined again by the small terms $\sim \Delta \gamma_{i}$ and $\sim\left(E / \gamma_{0}\right)^{2}$ in Eq. (68). This was particularly the case for armchair nanotubes, Figs. 12 and 13, where the translation unit's step corresponded to $\Delta n_{\|}=2$.

For the simplified model of the curvature-dependent transfer parameters used here ${ }^{52}$ the second term in Eq. (68) is negative for the whole range $0 \leqslant \theta \leqslant \pi / 6$ of the chiral angles. Therefore, as in the case of armchair tubes, we will see the competition between the second and the third terms in Eq. (68) in function of the transmission energy and the accompanying change of the period of oscillations of the slowly varying harmonic components in chiral tubes also. Indeed, Fig. 17 confirms that according to Eqs. (67) and (68) the transmission is almost energy independent at low $E$, the period of its oscillations as function of distance between the leads increases with $E$ becoming maximal at $E \sim \sqrt{\left|\Delta \gamma_{i} \gamma_{0}\right|}$ [see Eq. (61)], Fig. 17(c), and than starts to decrease [Fig. 17(d)]. This scenario, identical to the one found in armchair tubes (Fig. 12), is common for all primary metallic chiral tubes. The period of slow oscillations of the transmission function at low energies is mainly defined by the product of two major factors $\left(\gamma_{0} / f(\Delta \gamma)\right)$ and $\left(n^{\prime}+m^{\prime}\right) /\left(n^{\prime 2}+m^{\prime 2}\right.$ $\left.+n^{\prime} m^{\prime}\right)$, where $f(\Delta \gamma)$ denotes the expression from the brackets in the second term of Eq. (68). The first factor is proportional to $\left|\Delta \gamma_{i} / \gamma_{0}\right|$, i.e., to the square of the tube's diameter $^{20}$ and, for a given diameter, is minimal for armchair tubes. The second factor diminishes in tubes with large $n^{\prime}$ and $m^{\prime}$ which is merely the result of the increase of the translation unit $|\mathbf{T}|$ in such tubes (Table I).

Note that the above contribution to the oscillation of the transmission function at low energies is also responsible for the oscillation of the transmission in the gap region (Fig. 16). This is because in both cases the oscillation of the transmission is defined by the real part of $k_{\|}$corresponding to contributing poles. The last is practically the same for both the gap and the band region at low $E$, which is confirmed by close values of the period of oscillations of the transmission function in these two regions [Figs. 16 and 17(a)].

Let us investigate now the distance dependence of the transmission for other directions of the shift vector. To do so we write $\Delta \mathbf{t}$ in the form $p \Delta \mathbf{t}_{0}$, where $p$ is an integer and $\Delta \mathbf{t}_{0}$ is the smallest vector connecting equidistant carbon atoms in the direction of a chosen shift vector. With Table I we express $\Delta \mathbf{t}_{0}$ through the $\left(n_{\|}, n_{\perp}\right)$ coordinates and upon substitution of $\Delta \mathbf{t}$ into Eq. (67) we find the dependence of the transmission on $p$, the number of equidistant steps in the $\Delta \mathbf{t}$ direction. Actually to assess the character of this dependence it is enough to find the phase of the harmonic functions in Eq. (67) corresponding to the minimal shift in a given direction $\Delta \mathbf{t}_{0}$. Consider, for instance, the chiral tubes $(2 n, n)$. Taking a minimal step in the direction of the armchair line parallel to the $x$ axis [Fig. 1(a)], $\Delta \mathbf{t}_{0}=\mathbf{a}_{1}+\mathbf{a}_{2}$, we find the main contribution to the phase of the harmonic functions to be zero. This means that taking more steps in the same direction the phase of the harmonic functions will change slowly due to the second and the third small terms in Eq. (68). Hence the transmission is described by a single long period harmonic function very much as the transmission along the $\mathbf{T}$ vector (Fig. 17). Taking now a minimal step in the direction of a zigzag line parallel to the $y$ axis [Fig. 1(a)] $\Delta \mathbf{t}_{0}=\mathbf{a}_{1}-\mathbf{a}_{2}$, we find the main contribution to the arguments of harmonic functions to be equal to $4 \pi / 3$. Since this phase repeats after three steps the transmission function will be described by three slowly varying (as a result of small terms in $\tilde{\varphi}$ ) harmonic functions shifted equidistantly relative to each other by $2 \pi / 3$. As already discussed, the nonmonotonic change of the period of oscillations of the three harmonic components as function of $E$ results from the competition between small terms in Eq. (68). The two chosen directions are special since the resulting transmission function is described, respectively, by one and three harmonic components only. This is, however, not the case for most other directions. For instance, taking in the same nanotube $\Delta \mathbf{t}_{0}=\mathbf{a}_{1}$, i.e., aligned to another zigzag line, we obtain for the main contributions to the phases in Eq. (67) the value $13 \pi / 21$. This phase will repeat only after 21 steps in the direction of $\Delta \mathbf{t}_{0}$, which means that the corresponding transmission will be described by an equal number of shifted slowly varying harmonic functions. In arbitrary primary metallic tubes the number of harmonic components describing the transmission in a general direction of the shift vector can be as large as $n^{\prime 2}$ $+m^{\prime 2}+n^{\prime} m^{\prime}$ for tubes of the first kind $(R=3)$ and three times larger for tubes of the second kind $(R=1)$, Eq. (62). Precisely the same is true for the decaying transmission in the gap region, which oscillating behavior is determined by the value of $k_{\|}$at the upper band edge.

As for the amplitude of transmission, it is independent from the direction of the shift vector $\Delta \mathbf{t}$, which follows from the general form of Eq. (67). It is only weakly dependent on the transmission energy for relatively small values of $E$. Finally, it is practically independent from the chirality of the tubes but it is strongly dependent on their diameter. This is illustrated by the exact solutions for the transmission in the band region of zigzag and armchair tubes, Eqs. (54) and (60). Since the diameter $D$ of these tubes is related to $n$ as $n a / \pi$ and $\sqrt{3} n a / \pi$, respectively, we find from these equations that the amplitude of the transmission in the Fermi region (for relatively weak coupling to the leads) is given by a common formula 


$$
T \approx\left(\frac{\Delta}{\gamma_{0}} \frac{2 a}{\pi D}\right)^{2}
$$

which is also valid for arbitrary chiral nanotubes because zigzag and armchair tubes represent the two opposite limits of tube chirality.

Interestingly, the density of states in the Fermi region of single-wall carbon nanotubes was found to be independent from the chiral angle, separately for primary metallic and primary semiconducting tubes with roughly the same diameters, both in spectroscopy ${ }^{62-64}$ and in tight-binding calculations. ${ }^{65,66}$ The reason for this was found ${ }^{65}$ to be the circular conical structure of the two $\pi$ bands close to the Fermi points $K_{1,2}$. Extending this conclusion, we can state that the amplitude of the Green's function as a whole is almost independent from the chiral angle in this energy region. As a result the quantities deriving from $G^{0}$ are only dependent on the diameter of a given type of nanotube (i.e., primary metallic or primary semiconducting), as $D^{-1}$ for the density of states and $D^{-2}$ for the transmission, Eq. (69).

\section{DISCUSSION AND CONCLUSIONS}

\section{A. Features of the analytical approach}

We have investigated the transmission through sidecontacted straight carbon nanotubes for the case of monoatomic contacts within the Landauer-Bütikker formalism. An approach is proposed where the perturbed Green's function of the nanotube $G$ is expressed through the unperturbed one $G^{0}$ via the Dyson equation. This approach is especially convenient when few carbon atoms are in direct contact with the leads. $G^{0}$ corresponds to an isolated single-wall nanotube, long enough to be well approximated by an infinite tube, when the effect of the edge states can be neglected. Recent quantum chemistry calculations have shown that this condition is actually satisfied already for tubes of moderate length, e.g., 5-10 nm long. ${ }^{67}$

In the present paper an analytical expression for $G^{0}$ is derived for undistorted nanotubes within the tight-binding approach taking into account the effect of tube's curvature on the transfer parameters. This is achieved by the transformation of the hexagonal Brillouin zone of graphene into a rectangular one. In this new Brillouin zone, the folded band structure contains equidistant nanotube lines of equal length. Their number matches the greatest common divisor of the nanotube's indices $n$ and $m$. The matrix elements of $G^{0}$ represent a superposition of amplitudes corresponding to two one-dimensional $\pi$ bands of each nanotube line in the rectangular Brillouin zone. The contribution of each such band comes via $n^{\prime}+m^{\prime}$ poles $\left(n^{\prime}\right.$ and $m^{\prime}$ are reduced nanotube indices) corresponding to zeroes of a polynomial, Eq. (28), of a general form for all nanotubes. These zeroes are obtained analytically for zigzag and armchair tubes leading to simple expressions for $G^{0}$, Eqs. (B1)-(B7). For arbitrary chiral tubes these zeroes are found numerically and substituted into Eqs. (30) and (31), which thus offer a convenient frame for calculating $G^{0}$.

\section{B. Key results}

The main conclusions concerning the side-contacted transmission through undeformed single-wall nanotubes can be summarized as follows.

(1) The transmission amplitude $[\sim G$ in Eq. (33)] is a superposition of contributions from 1D bands of all nanotube lines. Fragments of these bands, confined by two consecutive extrema, can either give an oscillating nondecaying or an oscillating decaying contribution depending whether the transmission energy falls within the corresponding band region or not. In the latter case the oscillations of the decaying amplitude are described by $\operatorname{Re}\left\{k_{\|}\right\}$of the corresponding pole, the value of which is not far from $k_{\|}$at the closest edge (relative to the energy of transmission) of the band fragment. $\operatorname{Re}\left\{k_{\|}\right\}$coincides with $k_{\|}$at the closest edge of the fragment if this edge, actually a singularity point, corresponds to one of the symmetry points of the folded Brillouin zone of the nanotube. This is the case for all singularities in zigzag tubes, for all singularities of the second and third type and one singularity of the first type, lying on the nanotube line $l$ $=n / 2$, in armchair tubes (Fig. 14), and only for $\Gamma$ and $M_{1}$ $-M_{3}$ points in chiral tubes. As for singularities of the first type $(l \neq n / 2)$ in armchair tubes and most of the singularities which are nonsymmetric in chiral tubes, $\operatorname{Re}\left\{k_{\|}\right\}$will coincide with $k_{\|}$at one of them only when the transmission energy is in close vicinity. However, $\operatorname{Re}\left\{k_{\|}\right\}$will gradually deviate from this value when $E$ is changed leading to the variation of the period of oscillation of the corresponding contribution. This is in sharp contrast to the case of end-contacted nanotubes with reflectionless contacts where only two "metallic" bands contribute to the transmission with one quantum of conduction each $(T=2)$ in the whole Fermi region. ${ }^{2,9,21}$

(2) The transmission at Van Hove singularities is nonzero or zero depending on the interference between the amplitudes at different $\mathbf{k}_{s}$ points which belong to a given singularity. In the former case the transmission is $\sim 1$ for weak coupling to the leads, while in the second case it can correspond either to a split transmission peak or to a completely missing peak. The realization of one of these three situations as function of the relative positions of the leads and the type of singularity is governed by simple rules derived for every kind of nanotube.

At large axial distances between the leads the transmission at singularities is proportional to the square of the inverse distance, i.e., has the same distance dependence as found in long monoatomic chains. ${ }^{39}$ At the same time it scales as a square of the diameter $D$ of the tube and as an inverse square of coupling strength to the leads and the energy of a given singularity (measured from $E=0$ ). This provides an appreciable transmission at large distances between the leads already for monoatomic contacts. For instance, in $(6,-6)$ zigzag tubes, for $\left|\Delta / \gamma_{0}\right|=0.1$, the transmission at the first singularity (pinned at the Fermi level) is still $T$ $\approx 0.2$ at the distance of 2000 translational units. Moreover this transmission scales as $D^{6}$ at large distances between the leads (the corresponding energy is $D^{-2}$ ), i.e., increases very fast with the diameter of these tubes. 
In zigzag tubes $(n,-n)$ with even $n$ the nanotube line $l$ $=n / 2$ at the edge of the rectangular Brillouin zone contains two flat bands $E= \pm \gamma_{0}$, which correspond to a line of singularities (one-dimensional singular continuum). The transmission at these energies is only nonzero along separate zigzag chains. This transmission is close to unity, nondecaying with distance and independent from tube's diameter.

(3) In the gap region of semiconducting nanotubes the transmission decays exponentially with the axial distance between the leads, but much slower compared to monoatomic chains for the same energy deviation from the Fermi level. Therefore in this energy region the nanotubes are expected to be much better electronic guides than single band chains.

The transmission for these energies is of purely decaying type in zigzag tubes, but acquires in addition an oscillating character in chiral tubes, determined by the value of $k_{\|}$at the band edge. In the middle of the gap $E=0$, the transmission vanishes for contacted carbon atoms of the same type due to the symmetry of the band structure with respect to this energy point.

The lack of mirror symmetry planes, passing through carbon atoms perpendicular to the tube's axis, in all nanotubes except for armchair ones, makes the transmission in the axial direction highly anisotropic in these tubes. This means that fixing one of the leads at a given carbon atom will result in a nonzero transmission in one direction and in a much lower value of transmission in the opposite direction. The transmission anisotropy effect is stronger in the gap region than in the band one. It also increases with the chirality of the tubes being maximal in zigzag nanotubes, which show the transmission in the two directions differing by several orders of magnitude in the gap region.

(4) In the band region (not far from the Fermi level) the main contribution to the transmission comes from the nanotube lines intersecting (or passing close to) the Fermi points $K_{1,2}$. The zigzag tubes show oscillations of transmission only in the circumferential direction, while the armchair tubes show oscillations of $T$ only in the axial direction. In both cases the oscillations are fast, with the period of a few interatomic distances.

In addition, the oscillations in armchair tubes are modulated by long period harmonic components so that the transmission looks as a superposition of three equally shifted harmonic functions. The transmission in chiral tubes in different directions of a shift vector is generally a superposition of many such long period harmonic components. It is found that the primary metallic chiral tubes can be divided into two groups, with $n^{\prime}-m^{\prime}$ either divisible or not by $3,{ }^{42}$ showing qualitatively different behavior of the transmission function.

The long period oscillations of the transmission are determined by small corrections to the linear dispersion of the bands in the region of conical intersections at $K_{i}$ points. These involve the curvature induced modification of the transfer parameters and the quadratic terms in the dispersion law, both depending on the chirality of the tube. The competition between these contributions give rise to a nonmonotonic dependence of the period of slow oscillations of $T$ as function of the transmission energy.
The amplitude of the transmission is almost independent from chirality of the tubes but strongly depends on their diameter, as $D^{-2}$. This behavior has a common origin ${ }^{65}$ with the independence of density of states from the chiral angle in single-wall nanotubes of a given diameter observed in experiment. $^{62-64}$

The effect of the curvature on the transfer parameters was treated here via the misalignment of the atomic $p_{z}$ orbitals at nearest neighbor carbon atoms. ${ }^{52}$ This leads, in particular, to the decrease of the transfer parameter for a given pair of carbon atoms with the curvature of the tube's surface in the direction of the corresponding bond. This model does not include the accompanying change of the bond lengths ${ }^{68}$ and the $\sigma-\pi$ rehybridization effects, ${ }^{55,56}$ which have been claimed to increase the transfer parameters. ${ }^{56}$ While the question about the major reason for the curvature dependence of the transfer parameters is still open, ${ }^{69}$ one should mention that it would not change qualitatively the results presented here. In zigzag and armchair tubes the curvature effect is expressed via one single parameter $\Delta \gamma$ due to symmetry reasons. The change of sign of $\Delta \gamma$ merely reverses the direction of transmission anisotropy in zigzag tubes, while it modifies the behavior of the transmission in the Fermi region of armchair tubes, making the period of slow oscillations constantly decreasing when $|E|$ is increased (see Fig. 12). The same modification of transmission will emerge in chiral tubes also when the sign of all $\Delta \gamma_{i}$ is changed (see Fig. 17).

\section{Summary and outlook}

The detailed analysis of side-contacted transmission of the nanotubes shows a rich phenomenology already for the case of single-atomic contacts. Note, that the resulting transmission cannot exceed the value $T=1$, as it is expected in cases when at least one of the contacts is monoatomic and provides one single orbital for the transmission. ${ }^{70} \mathrm{We}$ envisage that direct experimental proof of the discussed effects should become possible by measurements involving leads thin enough, like STM tips. It was proven recently ${ }^{71}$ that such a technique as the tunneling spectroscopy is indeed capable to measure details of electronic structure of single-wall nanotubes in meV range. On the other hand an experimental setup using two STM tips on the same nanotube seems in principle feasible, although perhaps difficult to realize. However, with the current performance of STM devices, it is quite unprobably to achieve equal coupling to the nanotube for both leads. The theory is generalized straightforwardly to the case of differential coupling to the leads by expressing Eq. (33) as a product of two factors depending on $\Delta_{1}$ and $\Delta_{2}$, respectively. It is important to note that most of the obtained results will not change qualitatively in this case since they reflect the intrinsic properties of the nanotubes, i.e., are defined by $G^{0}$. For instance, the strong anisotropy of conductance in semiconducting tubes is such an intrinsic property, described by Eq. (C11), and will therefore show 
up for arbitrary coupling strength to the leads. For the same reasons we disregarded the energy dependence of the selfenergies, Eq. (32), in the present treatment.

A moderate increase of the number of contacted atoms is not expected to change qualitatively most of the results obtained for single-atomic contacts. The formalism developed in Sec. II is easily applied to this case too since it merely implies an additional inversion of the self-energy matrix in Eq. (10) having the dimension of the number of contacted carbon atoms in one lead. The transmission amplitude will obviously increase. In the limit of large number of contacted atoms the behavior of the transmission is qualitatively different, showing $k$-vector conservation effects for the case of uniform contacts ${ }^{27,28}$ and the saturation of the transmission with the length of the contact and/or the coupling strength to the leads. ${ }^{28}$

In order to keep the description as simple as possible, we did not consider in this paper the effects of interelectron interaction on the transmission, leading to charge redistribution close to the contacts ${ }^{72,73}$ and Luttinger liquid effects, ${ }^{74-76}$ as well as the structural distortions ${ }^{20-23}$ and the influence from impurities. ${ }^{11,12}$ These effects are always present at some extent in the conductivity of carbon nanotubes and their rationalization within an approach based on a detailed electronic structure is a challenge for the future investigations.

\section{ACKNOWLEDGMENTS}

This work was supported by the Flemish National Science Foundation (FWO) and the Belgian Government through the Concerted Action Scheme (GOA).

\section{APPENDIX A}

We prove here that the rectangular Brillouin zone in Fig. 2 defined by the vectors $\mathbf{W}_{\|}$and $\mathbf{W}_{\perp}$ in Eq. (18) is equivalent to the conventional first Brillouin zone of the graphene lattice in Fig. 1(b). The proof comprises two points. First, it can be checked straightforwardly that both Brillouin zones have the same area in the reciprocal space equal to $8 \pi^{2} / \sqrt{3} a^{2}$. Second, one can show that there are no equivalent points inside the rectangular Brillouin zone. Indeed, suppose the contrary, that there are two equivalent points inside this Brillouin zone separated by a lattice vector of the reciprocal Bravais lattice

$$
p \mathbf{b}_{1}+q \mathbf{b}_{2}=u \mathbf{W}_{\|}+v \mathbf{W}_{\perp},
$$

with $0<u<1$ and $0<v<1$ and $p, q$ some integer numbers. Making use of Eqs. (18) gives the following expressions for $p$ and $q$ :

$$
\begin{aligned}
& p=v \frac{2 n^{\prime}+m^{\prime}}{2\left(n^{\prime 2}+m^{\prime 2}+n^{\prime} m^{\prime}\right)}+u m^{\prime}, \\
& q=v \frac{n^{\prime}+2 m^{\prime}}{2\left(n^{\prime 2}+m^{\prime 2}+n^{\prime} m^{\prime}\right)}-u n^{\prime},
\end{aligned}
$$

where $n^{\prime}$ and $m^{\prime}$ are defined in Eq. (17). Combining Eqs. (A2) and (A3) yields

$$
n^{\prime} p+m^{\prime} q=v .
$$

Equation (A4) is never satisfied since the left-hand side is an integer while the right-hand side is always a noninteger number. This contradicts Eq. (A1) based on the assumption of existence of equivalent points in the rectangular Brillouin zone. Finally, since there are no equivalent points inside the rectangular Brillouin zone and on the other hand this zone is not smaller than the hexagonal Brillouin zone, it is necessarily equivalent to the latter.

\section{APPENDIX B}

Here we quote some analytical results for the Green's function of zigzag and armchair single-wall nanotubes. In zigzag nanotubes, the results are written in a more compact way for $(n,-n)$ tubes $\left(n_{\|}=-n_{x}, n_{\perp}=n_{y}\right)$; the orientation of the nanotube lines is shown in Fig. 2(a) and corresponds to $\gamma_{2}=\gamma_{3}$ [Fig. 1(a)]. In this case the solutions of the polynomial (28) are the following: ${ }^{47}$

$$
w_{ \pm}=\frac{z^{2}-\gamma_{1}^{2}-4 \gamma_{2}^{2} \cos ^{2} \frac{\pi l}{n} \pm D}{4 \gamma_{1} \gamma_{2} \cos \frac{l \pi}{n}},
$$

$$
\begin{gathered}
D=\sqrt{\left[z^{2}-\left(\gamma_{1}-2 \gamma_{2} \cos \frac{l \pi}{n}\right)^{2}\right]\left[z^{2}-\left(\gamma_{1}+2 \gamma_{2} \cos \frac{l \pi}{n}\right)^{2}\right]} \\
w_{+} w_{-}=1 .
\end{gathered}
$$

For $|E|<\sqrt{\gamma_{1}^{2}+4 \gamma_{2}^{2} \cos ^{2}(l \pi / n)}, w_{+}$will be inside the unit circle $|w|<1$, while $w_{-}$outside, and vice versa. Both solutions are on the unit circle (up to an infinitesimal shift arising from the imaginary part of $z$ ), when the energy falls into the corresponding band, $\left|\gamma_{1}-2 \gamma_{2} \cos (l \pi / n)\right|<|E|<\mid \gamma_{1}$ $+2 \gamma_{2} \cos (l \pi / n) \mid$.

Inserting Eq. (B1) into Eqs. (30), (31) we obtain the Green's functions for atoms of the same type:

$$
G^{0}(\Delta \mathbf{t}, 11 ; E)=-\frac{z}{n} \sum_{l} \cos \left(\frac{\pi l}{n} n_{\perp}\right) \frac{w_{ \pm}^{\left|n_{\|}\right|}}{ \pm D}
$$

and for atoms of different type

$$
\begin{aligned}
G^{0}(\Delta \mathbf{t}, 12 ; E)= & -\frac{1}{n} \sum_{l} \cos \left(\frac{\pi l}{n} n_{\perp}\right) \frac{w_{ \pm}^{\left|n_{\|}\right|}}{ \pm D} \\
& \times \begin{cases}\left(\gamma_{1}+2 \gamma_{2} \cos \frac{\pi l}{n} w_{ \pm}\right) & \text {if } n_{\|} \geqslant 0, \\
\left(\gamma_{1}+2 \gamma_{2} \cos \frac{\pi l}{n} w_{ \pm}^{-1}\right) & \text { if } n_{\|}<0,\end{cases}
\end{aligned}
$$

where the sign for $D$ corresponds to the subscript of $w$. 
In the case of $n$ even, the solution corresponding to the line $l=n / 2$ is obtained from $w$ in Eq. (B1) by following a limiting procedure and yields $w=0$ (there is only one solution because the second degree polynomial reduces to a first degree one in this special case). Then the corresponding term in the Green's function is the following:

$$
-\frac{1}{n} \cos \left(\frac{\pi}{2} n_{\perp}\right) \frac{\delta_{n_{\|}, 0}}{\gamma_{1}^{2}-z^{2}} \times \begin{cases}z & \text { for } G^{0}(\Delta \mathbf{t}, 11 ; E) \\ \gamma_{1} & \text { for } G^{0}(\Delta \mathbf{t}, 12 ; E) .\end{cases}
$$

Equations (B1)-(B4) show divergence of $G^{0}$ at singularity points described by Eq. (41).

For $(n, n)$ armchair nanotubes the solutions of the polynomial (28) are the following:

$$
\begin{gathered}
w_{i \pm}=-\left(x_{i} \pm \sqrt{x_{i}^{2}-1}\right) \\
x_{i}=\frac{1}{2\left|\gamma_{2}\right|}\left(\left|\gamma_{1}\right| \cos \frac{\pi l}{n} \pm \sqrt{z^{2}-\gamma_{1}^{2} \sin ^{2} \frac{\pi l}{n}}\right), \\
i=1,2, \\
w_{1-} w_{1+}=w_{2-} w_{2+}=1,
\end{gathered}
$$

where the upper and the lower sign in the second equation correspond to $i=1,2$, respectively. $w_{1+}$ is never inside the unit circle, then $w_{1-}$ is always inside. For $|l|=n / 2, w_{2+}$ is always inside the unit circle, while $w_{2-}$ is never inside. For $|l|<n / 2, w_{2+}$ is only inside the unit circle if $|E|>\left|\gamma_{1}\right|$, otherwise $w_{2-}$ is inside. The above solutions are situated on the unit circle (up to infinitesimal shifts due to the imaginary part of the energy) when $E$ falls in the following energy domains within the corresponding band: $\left|E_{l}^{1}\right|<|E|<\left|E_{l}^{2}\right|$ for $w_{1 \pm}$ and $\left|E_{l}^{1}\right|<|E|<\left|E_{l}^{3}\right|$ for $w_{2 \pm}$, where $E_{l}^{i}$ are defined in Eqs. (56).

Inserting Eq. (B5) into Eqs. (30), (31) for $G^{0}$, we obtain for atoms of the same type

$$
\begin{aligned}
G^{0}(\Delta \mathbf{t}, 11 ; E) \\
=-\frac{z}{n} \sum_{l} \cos \left(\frac{\pi l}{n} n_{\perp}\right) \frac{1}{2 \sqrt{z^{2}-\gamma_{1}^{2} \sin ^{2}(\pi l / n)}} \\
\quad \times \frac{1}{2\left|\gamma_{2}\right|}\left(-\frac{w_{1-}^{\mid n \|}}{\sqrt{x_{1}^{2}-1}} \mp \frac{w_{2 \pm}^{|n|}}{\sqrt{x_{2}^{2}-1}}\right),
\end{aligned}
$$

$G^{0}(\Delta \mathbf{t}, 12 ; E)$

$$
\begin{aligned}
& =-\frac{1}{n} \sum_{l} \exp \left(i \frac{\pi l}{n} n_{\perp}\right) \frac{1}{2 \sqrt{z^{2}-\gamma_{1}^{2} \sin ^{2} \frac{\pi l}{n}}} \\
& \quad \times \frac{1}{2\left|\gamma_{2}\right|}\left(-\frac{w_{1-}^{|n| \mid}\left(\gamma_{1}-2 x_{1} \gamma_{2} e^{-i(\pi l / n)}\right)}{\sqrt{x_{1}^{2}-1}}\right. \\
& \left.\mp \frac{w_{2 \pm}^{\mid n \|}\left(\gamma_{1}-2 x_{2} \gamma_{2} e^{-i(\pi l / n)}\right)}{\sqrt{x_{2}^{2}-1}}\right),
\end{aligned}
$$

where $n_{\|}=n_{y}$ and $n_{\perp}=n_{x}$ [see Eqs. (22)]. The Green's function becomes divergent at singularity points, Eqs. (56).

Note that $G^{0}(\Delta \mathbf{t}, 12 ; E)$ in Eq. (B7) has the same form for both $n_{\|} \geqslant 0$ and $n_{\|}<0$, which is not the case for zigzag and chiral tubes. This is because in armchair tubes all carbon atoms lie on the reflection planes $n_{\|} \rightarrow-n_{\|}$, perpendicular to the tube's axis.

In the case when the differences in the transfer parameters for nearest neighbor carbon atoms can be neglected, the above results for armchair nanotubes coincide with those obtained in Ref. 11. The Green's functions for ideal zigzag nanotubes in the same single transfer parameter approximation have been calculated in Ref. 48 for pairs of carbon sites oriented along the axis of the nanotube.

\section{APPENDIX C}

We give here the analytical expressions for transmission in the region close to the Fermi level of undoped zigzag nanotubes. First, $w$ in Eq. (B1) is rewritten in a more convenient form ${ }^{47}$

$$
w_{ \pm, l}=x_{l} \pm \sqrt{x_{l}^{2}-1},
$$

with

$$
x_{l}=\frac{z^{2}-\gamma_{1}^{2}-4 \gamma_{2}^{2} \cos ^{2} \frac{\pi l}{n}}{4 \gamma_{1} \gamma_{2} \cos \frac{\pi l}{n}} .
$$

It follows from the definition of $x_{l}$ that $\left|x_{l}\right| \leqslant 1$ for energies within the bands corresponding to the nanotube line $l$, and $\left|x_{l}\right|>1$ otherwise. In a wide region around the Fermi level $|E|<\left|\gamma_{0}\right|$, the "metallic" line $l= \pm n / 3$ gives the main contribution to $G^{0}$ in Eqs. (B2) and (B3) due to a small denominator $D$, Eq. (B1). For primary metallic tubes we have for the contributing pole in a good approximation

$$
w_{+, n / 3} \approx \begin{cases}-e^{-\sqrt{\Delta \gamma^{2}-E^{2}} /\left|\gamma_{0}\right|}, & |E|<\Delta \gamma, \\ -e^{i \operatorname{sgn}(E) \sqrt{E^{2}-\Delta \gamma^{2}} /\left|\gamma_{0}\right|}, & \Delta \gamma<|E|<\left|\gamma_{0}\right|,\end{cases}
$$

and for atoms of different type 
where $\Delta \gamma=\left|\gamma_{1}-\gamma_{2}\right|$. One can see that $w_{+, n / 3} \rightarrow-1$ when $E$ approaches the first singularity $(E= \pm \Delta \gamma)$, as expected.

Inserting Eq. (C3) into Eqs. (B2) and (B3) we obtain for the gap region $|E|<\Delta \gamma$

$$
\begin{aligned}
G^{0}(\Delta \mathbf{t}, i j ; E)= & -\frac{g_{i j}}{n\left|\gamma_{0}\right|} \cos \left(\frac{\pi}{3} n_{\perp}\right)(-1)^{\left|n_{\|}\right|} \\
& \times e^{-\left(\sqrt{\Delta \gamma^{2}-E^{2}} /\left|\gamma_{0}\right|\right)\left|n_{\|}\right|},
\end{aligned}
$$

where the $g_{i j}$ 's are defined as follows:

$$
\begin{gathered}
g_{11} \approx \frac{E}{\sqrt{\Delta \gamma^{2}-E^{2}}}\left(1+\frac{\Delta \gamma}{2\left|\gamma_{0}\right|}\right), \\
g_{12} \approx \operatorname{sgn}(\gamma)\left(\frac{\Delta \gamma}{\sqrt{\Delta \gamma^{2}-E^{2}}} \pm 1-\frac{1}{2} \frac{\sqrt{\Delta \gamma^{2}-E^{2}}}{\left|\gamma_{0}\right|}+\frac{\Delta \gamma}{2\left|\gamma_{0}\right|}\right), \\
\begin{cases}+, & n_{\|} \geqslant 0, \\
-, & n_{\|}<0 .\end{cases}
\end{gathered}
$$

With Eqs. (C4) and (C5) we obtain for the transmission through carbon atoms of type $i$ and $j$ :

$$
T_{i j}=\frac{\frac{4 \Delta^{2}}{n^{2} \gamma_{0}^{2}} g_{i j}^{2} \cos ^{2}\left(\frac{\pi}{3} n_{\perp}\right) e^{-2\left(\sqrt{\Delta \gamma^{2}-E^{2}} /\left|\gamma_{0}\right|\right)\left|n_{\|}\right|}}{\left|\left(1-\frac{i \Delta}{n\left|\gamma_{0}\right|} g_{11}\right)^{2}+\frac{\Delta^{2}}{n^{2} \gamma_{0}^{2}} g_{i j}^{2} \cos ^{2}\left(\frac{\pi}{3} n_{\perp}\right) e^{-2\left(\sqrt{\Delta \gamma^{2}-E^{2}} / \mid \gamma_{0}\left\|n_{\|}\right\|\right.}\right|^{2}} .
$$

Similarly, we obtain for the band region close to the Fermi level $\Delta \gamma<|E|<\left|\gamma_{0}\right|$,

$$
\begin{aligned}
G^{0}(\Delta \mathbf{t}, i j ; E)= & -\frac{i}{n\left|\gamma_{0}\right|} \frac{h_{i j}}{\sqrt{1-\left(E / 2 \gamma_{0}\right)^{2}}} \cos \left(\frac{\pi}{3} n_{\perp}\right) \\
& (-1)^{\mid n \|} e^{i \operatorname{sgn}(E)\left(\sqrt{E^{2}-\Delta \gamma^{2}} /\left|\gamma_{0}\right|\right) \mid n \|},
\end{aligned}
$$

where the $h_{i j}$ 's are as follows:

$$
\begin{gathered}
h_{11} \approx \frac{|E|}{\sqrt{E^{2}-\Delta \gamma^{2}}}, \\
h_{12} \approx \operatorname{sgn}(\gamma)\left(\operatorname{sgn}(E) \frac{\Delta \gamma}{\sqrt{E^{2}-\Delta \gamma^{2}}} \mp i\right), \begin{cases}-, & n_{\|} \geqslant 0, \\
+, & n_{\|}<0,\end{cases}
\end{gathered}
$$

and for the transmission through carbon atoms of type $i$ and $j$ in this region

$$
T_{i j}=\frac{\frac{4 \Delta^{2}}{n^{2} \gamma_{0}^{2}} \frac{\left|h_{i j}\right|^{2}}{1-\left(E / 2 \gamma_{0}\right)^{2}} \cos ^{2}\left(\frac{\pi}{3} n_{\perp}\right)}{\mid\left(1+\frac{\Delta}{n\left|\gamma_{0}\right|} \frac{h_{11}}{\sqrt{1-\left(E / 2 \gamma_{0}\right)^{2}}}\right)^{2}-\frac{\Delta^{2}}{n^{2} \gamma_{0}^{2}} \frac{h_{i j}^{2}}{1-\left(E / 2 \gamma_{0}\right)^{2}} \cos ^{2}\left(\frac{\pi}{3} n_{\perp}\right) e^{2 i \operatorname{sgn}(E)\left(\sqrt{E^{2}-\Delta \gamma^{2}} /\left|\gamma_{0}\right|\right)|n \||^{2}}} .
$$

At $E=0$, the contributions from the "metallic" line are obtained without approximation [see Eqs. (C3)-(C5)]:

$$
\begin{gathered}
w_{+, n / 3}=-\frac{\gamma_{2}}{\gamma_{1}}, \\
\begin{cases}G^{0}(\Delta \mathbf{t}, 12 ; 0)=-\frac{2}{n \gamma_{1}} \cos \left(\frac{\pi}{3} n_{\perp}\right)\left(-\frac{\gamma_{2}}{\gamma_{1}}\right)^{|n \||}, & n_{\|} \geqslant 0, \\
G^{0}(\Delta \mathbf{t}, 12 ; 0)=0, & n_{\|}<0,\end{cases}
\end{gathered}
$$

These coincide with the general approximate solutions in Eqs. (C3) $-(C 5)$ up to terms of second order in $\Delta \gamma /\left|\gamma_{0}\right|$. For the opposite relationship between transfer parameters, $\left|\gamma_{1}\right|$ $<\left|\gamma_{2}\right|$ we obtain

$$
w_{+, n / 3}=-\frac{\gamma_{1}}{\gamma_{2}},
$$

$$
\begin{cases}G^{0}(\Delta \mathbf{t}, 12 ; 0)=0, & n_{\|} \geqslant 0, \\ G^{0}(\Delta \mathbf{t}, 12 ; 0)=\frac{2}{n \gamma_{1}} \cos \left(\frac{\pi}{3} n_{\perp}\right)\left(-\frac{\gamma_{1}}{\gamma_{2}}\right)^{\mid n \|}, & n_{\|}<0 .\end{cases}
$$


At the same time $G^{0}(\Delta \mathbf{t}, 11 ; 0)$ is always zero due to symmetry reasons (see Sec. III B).

\section{APPENDIX D}

We quote here some analytical results for the Green's function of armchair nanotubes in the energy region close to the Fermi level, $0 \leqslant|E|<\left|E_{ \pm 1}^{1}\right|$. Only $w_{1-, l}$ and $w_{2-, l}$ contribute substantially to $G^{0}$ in Eqs. (B6) and (B7) in this energy region.

For the "metallic" nanotube line, $l=0$, we find in a good approximation

$$
\begin{aligned}
w_{1(2)-, 0}= & -\exp \left\{i \operatorname { s g n } ( E ) \left[\frac{\pi}{3} \mp \frac{1}{\sqrt{3}}\left|\frac{E}{\gamma_{0}}\right|+\frac{\Delta \gamma}{\sqrt{3}\left|\gamma_{0}\right|}\right.\right. \\
& \left.\left.-\frac{1}{6 \sqrt{3}}\left(\frac{E}{\gamma_{0}}\right)^{2}\right]\right\},
\end{aligned}
$$

where the two signs in the exponent correspond to $w_{1-, 0}$ and $w_{2-, 0}$. Inserting these quantities into Eqs. (B6) and (B7) we find the following contribution of the "metallic" nanotube line to $G^{0}$ in this energy region

$$
\begin{aligned}
G^{0}(\Delta \mathbf{t}, 11 ; E)= & -\frac{i}{n\left|\gamma_{2}\right|} \frac{1}{\sqrt{3}}(-1)^{\mid n \|} \exp \left\{i \frac{1}{\sqrt{3}}\left(1-\frac{2 \Delta \gamma}{3\left|\gamma_{0}\right|}\right)\right. \\
& \left.\times \frac{E}{\left|\gamma_{0}\right|}\left|n_{\|}\right|\right\}\left[c_{1} \cos \left(\varphi n_{\|}\right)+i c_{2} \sin \left(\varphi\left|n_{\|}\right|\right)\right], \\
G^{0}(\Delta \mathbf{t}, 12 ; E)= & -\frac{i \operatorname{sgn}(\gamma)}{n\left|\gamma_{2}\right|} \frac{1}{\sqrt{3}}(-1)^{\mid n \|} \exp \left\{i \frac{1}{\sqrt{3}}(1\right. \\
& \left.\left.-\frac{2 \Delta \gamma}{3\left|\gamma_{0}\right|}\right) \frac{E}{\left|\gamma_{0}\right|}\left|n_{\|}\right|\right\}\left[c_{2} \cos \left(\varphi n_{\|}\right)\right. \\
& \left.+i c_{1} \sin \left(\varphi\left|n_{\|}\right|\right)\right],
\end{aligned}
$$

where

$$
\begin{gathered}
\varphi=\frac{\pi}{3}+\frac{1}{\sqrt{3}}\left[\frac{\Delta \gamma}{\left|\gamma_{0}\right|}-\frac{1}{6}\left(\frac{E}{\gamma_{0}}\right)^{2}\right], \\
c_{1}=1-\frac{\Delta \gamma}{3\left|\gamma_{0}\right|}+\left(\frac{E}{\gamma_{0}}\right)^{2}, \\
c_{2}=\frac{E}{\left|\gamma_{0}\right|}\left(-\frac{1}{3}+\frac{2 \Delta \gamma}{3\left|\gamma_{0}\right|}\right) .
\end{gathered}
$$

Note the independence of the $l=0$ contribution in Eq. (D2) from $n_{\perp}$.

Among other contributions to the Green's function, the largest ones come from the nanotube lines $l= \pm 1$, for which we have

$$
w_{1(2)-, 1}=-e^{\mp i \operatorname{sgn}(E) \varphi^{\prime}-y[1+(1 / 3) x]},
$$

$$
\varphi^{\prime}=\frac{\pi}{3}-\frac{x}{\sqrt{3}}+\frac{y^{2}}{6 \sqrt{3}}
$$

where the two signs in the exponent of the first equation correspond to $w_{1-, 1}$ and $w_{2-, 1}$. The parameters $x$ and $y$ are the following:

$$
\begin{gathered}
x=\left(1-\frac{\Delta \gamma}{\left|\gamma_{0}\right|}\right) \cos \frac{\pi}{n}-1, \\
y=\left(1-\frac{\Delta \gamma}{\left|\gamma_{0}\right|}\right) \sin \frac{\pi}{n} \sqrt{1-\epsilon^{2}},
\end{gathered}
$$

and a dimensionless energy variable

$$
\epsilon=\frac{|E|}{\left|\gamma_{1}\right| \sin \frac{\pi}{n}},
$$

have been introduced, $0 \leqslant \epsilon<1$. Inserting Eq. (D4) into Eqs. (B6) and (B7) we obtain the propagation amplitudes from these lines:

$$
\begin{aligned}
G^{0}(\Delta \mathbf{t}, 11 ; E)= & -\frac{E}{n \gamma_{2}^{2}} \frac{2}{\sqrt{3} y}(-1)^{n \|} e^{-y[1+(1 / 3) x]\left|n_{\|}\right|} \\
& \times \cos \frac{\pi}{n} n_{\perp}\left(c_{1}^{\prime} \cos \varphi^{\prime} n_{\|}+c_{2}^{\prime} \sin \varphi^{\prime}\left|n_{\|}\right|\right), \\
G^{0}(\Delta \mathbf{t}, 12 ; E)= & -\frac{\operatorname{sgn}(\gamma)}{n\left|\gamma_{2}\right|} \frac{2}{\sqrt{3} y}(-1)^{n} \| e^{-y[1+(1 / 3) x]\left|n_{\|}\right|} \\
& \times\left[(1+x)\left(\cos \frac{\pi}{n} n_{\perp}-\cos \frac{\pi}{n}\left(n_{\perp}-1\right)\right)\right. \\
& \times\left(c_{1}^{\prime} \cos \varphi^{\prime} n_{\|}+c_{2}^{\prime} \sin \varphi^{\prime} \mid n_{\|} \|\right) \\
& +y \cos \frac{\pi}{n}\left(n_{\perp}-1\right)\left(-c_{1}^{\prime} \sin \varphi^{\prime}\left|n_{\|}\right|\right. \\
& \left.\left.+c_{2}^{\prime} \cos \varphi^{\prime} n_{\|}\right)\right],
\end{aligned}
$$

where

$$
\begin{aligned}
& c_{1}^{\prime}=1+\frac{2}{3} x-\frac{1}{2} y^{2}, \\
& c_{2}^{\prime}=\frac{1}{6} y\left(2 x-y^{2}\right) .
\end{aligned}
$$


*Electronic address: Liviu.Chibotaru@chem.kuleuven.ac.be

${ }^{1}$ L. Langer, L. Stockman, J.P. Heremans, V. Bayot, C.H. Olk, C. Van Haesendonck, Y. Bruynseraede, and J.-P. Issi, J. Mater. Res. 9, 927 (1994).

${ }^{2}$ S.J. Tans, M.H. Devoret, H. Dai, A. Thess, R.E. Smalley, L.J. Georliga, and C. Dekker, Nature (London) 386, 474 (1997).

${ }^{3}$ S. Frank, P. Poncharal, Z.L. Wang, and W.A. de Heer, Science 280, 1744 (1998).

${ }^{4}$ A. Bachtold, C. Strunk, J.P. Salvetat, J.M. Bonard, L. Forro, T. Nussbaumer, and C. Schonenberger, Nature (London) 397, 673 (1999).

${ }^{5}$ W. Tian and S. Datta, Phys. Rev. B 49, 5097 (1994).

${ }^{6}$ R. Saito, G. Dresselhaus, and M.S. Dresselhaus, Phys. Rev. B 53, 2044 (1996).

${ }^{7}$ L. Chico, L.X. Benedict, S.G. Louie, and M.L. Cohen, Phys. Rev. B 54, 2600 (1996).

${ }^{8}$ R. Tamura and M. Tsukada, Phys. Rev. B 55, 4991 (1997).

${ }^{9}$ M.P. Anantram and T.R. Govindan, Phys. Rev. B 58, 4882 (1998).

${ }^{10}$ T. Ando and T. Nakanishi, J. Phys. Soc. Jpn. 67, 1704 (1998).

${ }^{11}$ H.J. Choi and J. Ihm, Solid State Commun. 111, 385 (1999).

${ }^{12}$ T. Kostyrko, M. Bartkowiak, and G.D. Mahan, Phys. Rev. B 59, 3241 (1999).

${ }^{13}$ S. Sanvito, Y.-K. Kwon, D. Tomanek, and C.J. Lambert, Phys. Rev. Lett. 84, 1974 (2000).

${ }^{14}$ C. Dekker, Phys. Today 52, 22 (1999).

${ }^{15}$ C. Schonenberger, A. Bachtold, C. Strunk, J.P. Salvetat, and L. Forro, Appl. Phys. A: Mater. Sci. Process. 69, 283 (1999).

${ }^{16}$ Z. Yao, C. Dekker, and P. Avouris, Carbon Nanotubes 80, 147 (2001).

${ }^{17}$ D.H. Robertson, D.W. Brenner, and J.W. Mintmire, Phys. Rev. B 45, 12592 (1992).

${ }^{18}$ M.M.J. Treacy, T.W. Ebbesen, and J.M. Gibson, Nature (London) 381, 678 (1996).

${ }^{19}$ M.R. Falvo, G.J. Clary, R.M. Taylor II, V. Chi, F.P. Brooks, Jr., S. Washburn, and R. Superfine, Nature (London) 389, 582 (1997).

${ }^{20}$ C.L. Kane and E.J. Mele, Phys. Rev. Lett. 78, 1932 (1997).

${ }^{21}$ M.B. Nardelli, Phys. Rev. B 60, 7828 (1999).

${ }^{22}$ A. Rochefort, F. Lesage, D.R. Salahub, and Ph. Avouris, Phys. Rev. B 60, 13824 (1999).

${ }^{23}$ L.F. Chibotaru, S.A. Bovin, and A. Ceulemans, Phys. Rev. B 66, 161401(R) (2002).

${ }^{24}$ C.T. White and T.N. Todorov, Nature (London) 393, 240 (1998).

${ }^{25}$ S. Compernolle, L.F. Chibotaru, and A. Ceulemans, J. Chem. Phys. 119, 2854 (2003).

${ }^{26}$ R. Landauer, Philos. Mag. 21, 863 (1970).

${ }^{27}$ J. Tersoff, Appl. Phys. Lett. 74, 2122 (1999).

${ }^{28}$ M.P. Anantram, S. Datta, and Y. Xue, Phys. Rev. B 61, 14219 (2000).

${ }^{29}$ Special issue on carbon nanotubes, Acc. Chem. Res. 35, 997 (2002).

${ }^{30}$ G. Fagas, G. Cuniberti, and K. Richter, Phys. Rev. B 63, 045416 (2001).

${ }^{31}$ D. Orlikowski, H. Mehrez, J. Taylor, H. Guo, and J. Wang, Phys. Rev. B 63, 155412 (2001).

${ }^{32}$ M.P. Anantram and T.R. Govindan, Phys. Rev. B 61, 5020 (2000).

${ }^{33}$ A. Rochefort and Ph. Avouris, Phys. Rev. B 60, 13824 (1999); J. Phys. Chem. A 104, 9807 (2000).

${ }^{34}$ M.S. Dresselhaus, G. Dresselhaus, and P.C. Eklund, Science of
Fullerenes and Carbon Nanotubes (Academic, New York, 1995).

${ }^{35}$ P.R. Wallace, Phys. Rev. 71, 622 (1947).

${ }^{36}$ S. Datta, Electronic Transport in Mesoscopic Systems (Cambridge University Press, Cambridge, 1995).

${ }^{37}$ W. Tian, S. Datta, S. Hong, R. Reifenberger, J.I. Henderson, and P. Kubiak, J. Chem. Phys. 109, 2874 (1998).

${ }^{38}$ D.A. Papaconstantopoulos, Handbook of the Band Structure of Elemental Solids (Plenum, New York, 1986).

${ }^{39}$ V. Mujica, M. Kemp, and M.A. Ratner, J. Chem. Phys. 101, 6856 (1994).

${ }^{40}$ P.W. Anderson, Phys. Rev. 124, 41 (1961); D.M. Newns, ibid. 178, 1123 (1969).

${ }^{41}$ E.N. Economou, Green's Functions in Quantum Physics (Springer-Verlag, Berlin, 1979).

${ }^{42}$ M. Damnjanović, I. Milošević, T. Vucović, and R. Sredanović, Phys. Rev. B 60, 2728 (1999).

${ }^{43}$ M. Damnjanović, T. Vucović, and I. Milošević, J. Phys. A 33, 6561 (2000).

${ }^{44}$ Z. Parra-Mejías, S. Compernolle, L.F. Chibotaru, and A. Ceulemans, in Electronic Properties of Molecular Nanostructures: XV International Winterschool/Euroconference, edited by Hans Kuzmany et al., AIP Conf. Proc. No. 591 (AIP, Melville, 2001), p. 442.

${ }^{45}$ A. Ceulemans, L.F. Chibotaru, S.A. Bovin, and P.W. Fowler, J. Chem. Phys. 112, 4271 (2000).

${ }^{46}$ M.S. Ferreira, J. d'Albuquerque e Castro, D.M. Edwards, and J. Mathon, J. Phys.: Condens. Matter 8, 11259 (1996).

${ }^{47}$ We use a convention for the square root, according to which the imaginary part of $\sqrt{-A^{2}+i \eta}$ has the same sign as $\eta$ ( $A$ and $\eta$ are real).

${ }^{48}$ M.S. Ferreira, T.G. Dargam, R.B. Muniz, and A. Latgé, Phys. Rev. B 62, 16040 (2000).

${ }^{49} \mathrm{~S}$. Wolfram, MATHEMATiCA ${ }^{\text {TM}}$ : A System for Doing Mathematics by Computer (Addison-Wesley, New York, 1988).

${ }^{50}$ J. Tersoff and D.R. Hamann, Phys. Rev. Lett. 50, 1998 (1983).

${ }^{51}$ V. Meunier and Ph. Lambin, Phys. Rev. Lett. 81, 5588 (1998).

${ }^{52}$ We suppose here for simplicity that the curvature dependence of the transfer parameters merely arises from the misalignment of the $\pi$ orbitals on the nearest neighbor carbon atoms (Ref. 20,53): $\gamma_{i}=\gamma_{0} \cos \phi_{i} ; \phi_{i}$ is the misalignment angle which can be expressed (Ref. 58) through the chiral angle $\theta$ for each pair $i$.

${ }^{53}$ A. Kleiner and S. Eggert, Phys. Rev. B 63, 073408 (2001).

${ }^{54}$ Note that strict mirror symmetry of the band structure with respect to $E=0$ only arises within the orthogonal single band tight-binding model used here. Different hybridization of the $\sigma$ bands to the bonding and antibonding $\pi$ band due to the curvature of the nanotubes surface (Ref. 55) and an explicit account of overlap of atomic $\pi$ orbitals at neighbor sites, included in more realistic non-orthogonal tight-binding-like models (Ref. 56) and DFT calculations (Ref. 57), will remove this symmetry.

${ }^{55}$ X. Blase, L.X. Benedict, E.L. Shirley, and S.G. Louie, Phys. Rev. Lett. 72, 1878 (1994).

${ }^{56}$ N. Hamada, S. Sawada, and A. Oshiyama, Phys. Rev. Lett. 68, 1579 (1992).

${ }^{57}$ J.W. Mintmire, B.I. Dunlap, and C.T. White, Phys. Rev. Lett. 68, 631 (1992).

${ }^{58}$ M.-F. Lin, R.-B. Chen, and F.-L. Shyu, Solid State Commun. 107, 227 (1998). 
${ }^{59}$ E.G. Emberly and G. Kirczenow, J. Phys.: Condens. Matter 11, 6911 (1999).

${ }^{60}$ The curvature-induced shift of the $K_{1,2}$ points corresponding to the intersection of the two $\pi$ bands is found from the condition (Ref. 20) $\sum_{i=1}^{3} \Delta \gamma_{i} \exp \left(i \mathbf{k} \cdot \mathbf{R}_{i}\right)=0$, which for armchair nanotubes gives $\Delta k_{\|}= \pm(2 / \sqrt{3} a)\left(\Delta \gamma /\left|\gamma_{0}\right|\right), \Delta k_{\perp}=0$.

${ }^{61}$ More exactly, the criterion is $\Delta G^{0}(\mathbf{0}, 11 ; E)_{l= \pm 1} \sim 1$, i.e., it is also determined by the coupling strength to the leads $\Delta$ (the subscript means the $l= \pm 1$ contribution).

${ }^{62}$ M.S. Dresselhaus, Nature (London) 391, 19 (1998).

${ }^{63}$ J.W.G. Wildöer, L.C. Venema, A.G. Rinzler, R.E. Smalley, and C. Dekker, Nature (London) 391, 59 (1998).

${ }^{64}$ T.W. Odom, J.-L. Huang, P. Kim, and C.M. Lieber, Nature (London) 391, 62 (1998).

${ }^{65}$ C.T. White and J.W. Mintmire, Nature (London) 394, 29 (1998).

${ }^{66}$ J.-C. Charlier and P. Lambin, Phys. Rev. B 57, R15 037 (1998).
${ }^{67}$ A. Rochefort, D.R. Salahub, and Ph. Avouris, J. Phys. Chem. B 103, 641 (1999).

${ }^{68}$ L. Yang and J. Han, Phys. Rev. Lett. 85, 154 (2000).

${ }^{69}$ A. Kleiner and S. Eggert, Phys. Rev. B 64, 113402 (2001).

${ }^{70}$ J. Heurich, J.C. Cuevas, W. Wenzel, and G. Schön, Phys. Rev. Lett. 88, 256803 (2002).

${ }^{71}$ M. Ouyang, J.-L. Huang, C.L. Cheung, and C.M. Lieber, Science 292, 702 (2001).

${ }^{72}$ A.A. Odintsov and Y. Tokura, Physica B 280, 386 (2000).

${ }^{73}$ K. Esfarjani, A.A. Farajian, Y. Hashi, and Y. Kawazoe, Appl. Phys. Lett. 74, 79 (1999).

${ }^{74}$ R. Egger and A.O. Gogolin, Phys. Rev. Lett. 79, 5082 (1997).

${ }^{75}$ C.L. Kane, L. Balents, and M.P.A. Fisher, Phys. Rev. Lett. 79, 5086 (1997).

${ }^{76}$ S.J. Tans, M.H. Devoret, R.J.A. Groeneveld, and C. Dekker, Nature (London) 394, 761 (1998). 